Louisiana State University

LSU Digital Commons

Faculty Publications

Department of Chemistry

$1-1-2016$

\title{
Tip enhanced laser ablation sample transfer for mass spectrometry
}

Kermit K. Murray

Louisiana State University

Suman Ghorai

Louisiana State University

Chinthaka A. Seneviratne

Louisiana State University

Follow this and additional works at: https://digitalcommons.Isu.edu/chemistry_pubs

\section{Recommended Citation}

Murray, K., Ghorai, S., \& Seneviratne, C. (2016). Tip enhanced laser ablation sample transfer for mass spectrometry. Materials Research Society Symposium Proceedings, 1754, 87-96. https://doi.org/10.1557/ opl.2015.286

This Conference Proceeding is brought to you for free and open access by the Department of Chemistry at LSU Digital Commons. It has been accepted for inclusion in Faculty Publications by an authorized administrator of LSU Digital Commons. For more information, please contact ir@lsu.edu. 


\section{Louisiana State University}

\section{LSU Digital Commons}

2016

\section{Tip-Enhanced Laser Ablation Sample Transfer for Mass Spectrometry}

Chinthaka Aravinda Seneviratne

Louisiana State University and Agricultural and Mechanical College, csenev1@1su.edu

Follow this and additional works at: https://digitalcommons.lsu.edu/gradschool_dissertations

Part of the Chemistry Commons

\section{Recommended Citation}

Seneviratne, Chinthaka Aravinda, "Tip-Enhanced Laser Ablation Sample Transfer for Mass Spectrometry" (2016). LSU Doctoral Dissertations. 2355.

https://digitalcommons.lsu.edu/gradschool_dissertations/2355 


\title{
TIP-ENHANCED LASER ABLATION SAMPLE TRANSFER FOR MASS SPECTROMETRY
}

\author{
A Dissertation \\ Submitted to the Graduate Faculty of the \\ Louisiana State University and \\ Agricultural and Mechanical College \\ in partial fulfillment of the \\ requirements for the degree of \\ Doctor of Philosophy \\ in \\ The Department of Chemistry \\ by \\ Chinthaka Aravinda Seneviratne \\ M.S., Bucknell University, 2011 \\ B.Sc., University of Kelaniya, 2008 \\ May 2016
}


This dissertation is dedicated

with love to my parents, Hector and Dayani Seneviratne my wife Sameera Herath and my little buddy Julian!. 


\section{ACKNOWLEDGEMENTS}

First and foremost, I give glory to God. Without God's blessings, none of this would have been possible.

It is a great pleasure to offer my unbounding appreciation for all who have supported and guided me over the course my doctoral studies to the completion of this dissertation. I would like to thank my doctoral mentor, Dr. Kermit K. Murray for his unwavering guidance and constant encouragement. Thank you for sharing your knowledge and allowing me the opportunity to conduct research in the lab, while averting me from taking wrong turns. I am extremely proud to contribute to the research work in your group and forever thankful for all you have done for me. I would also like to thank my doctoral committee members, Dr. George G. Stanley, Dr. Louis Haber, and Dr. Kenneth Matthews for their support and constructive advice.

A warm thank goes to the talented group of scientists both past and present in the Murray research group. All of you have helped me greatly in this journey, and I wish you all the very best in life.

I would also like to express my gratitude to all the faculty and staff in the Department of Chemistry at LSU, especially to Ms. Connie David and Dr. Jeonghoon Lee at LSU mass spectrometry facility. Thank you for being extremely helpful in my research work.

On a personal front, I take this opportunity to thank my wife, Sameera for her love, devotion, encouragement and for everything we have shared in life together. There are not enough words in me to express how grateful I am for all you have done, especially, taking care

of our most precious gift Julian and releasing me from all duties. I wanted you to know this accomplishment was not only for me, but was for us.

I am forever grateful to my wonderful parents and my siblings for a perfect upbringing in a perfect family with such love and care. You have always been my biggest encouragement. Thank you for all the sacrifices you've made for me to be the person I am today. 


\section{TABLE OF CONTENTS}

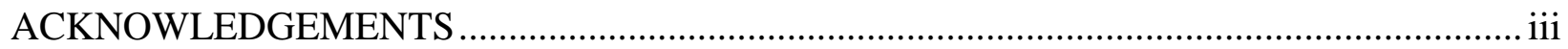

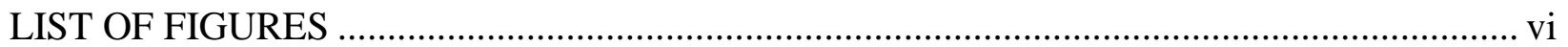

LIST OF ABBREVIATIONS AND SYMBOLS ................................................................ ix

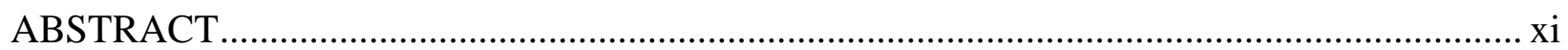

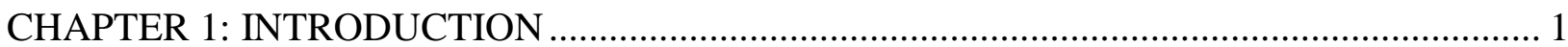

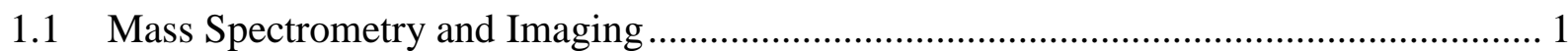

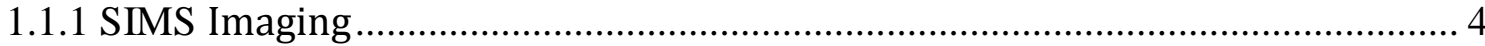

1.1.2 Laser Desorption Ionization Imaging …………................................................ 5

1.1.3 Ambient Sampling and Imaging Mass Spectrometry ....................................... 11

1.2 Near-Field Sampling and Imaging Mass Spectrometry …………................................. 18

1.2.1 Near-Field Microscopy.................................................................................. 18

1.2.2 Near-Field Ablation and Mass Spectrometry ………………………………....... 23

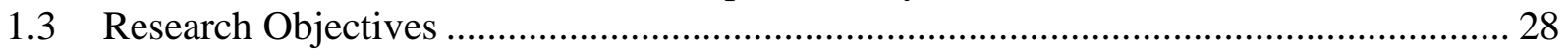

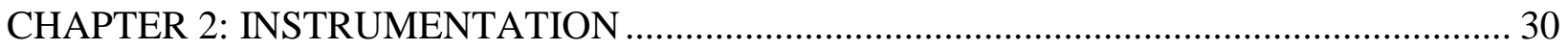

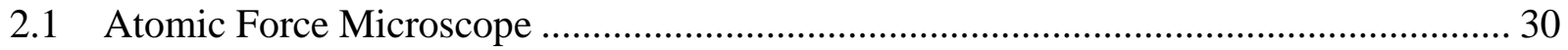

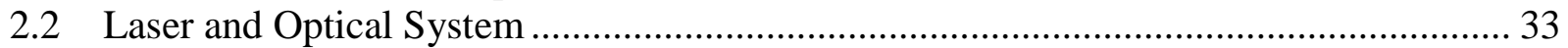

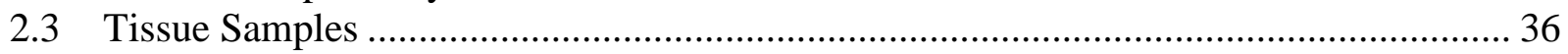

CHAPTER 3: TIP-ENHANCED LASER ABLATION SAMPLE TRANSFER FOR SMALL

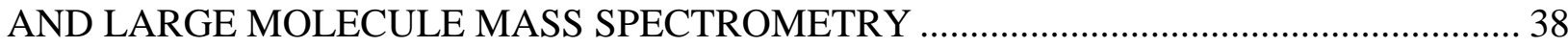

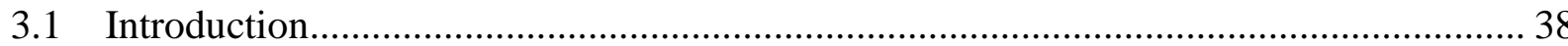

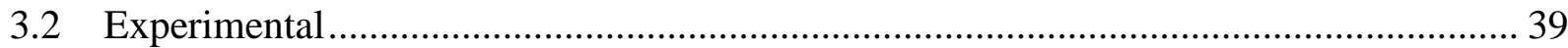

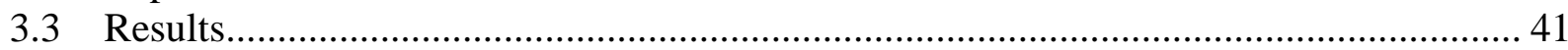

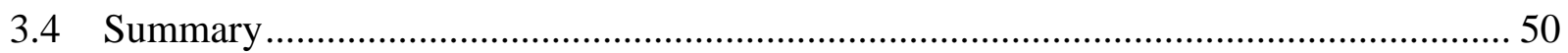

CHAPTER 4: TIP-ENHANCED LASER ABLATION SAMPLE TRANSFER FOR LARGE BIOMOLECULE AND TISSUE MASS SPECTROMETRY …………………………............ 52

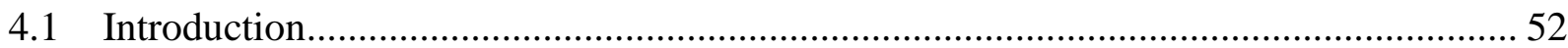

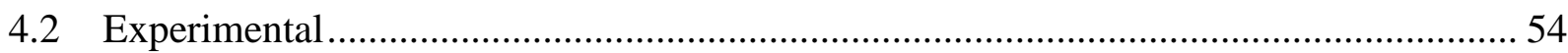

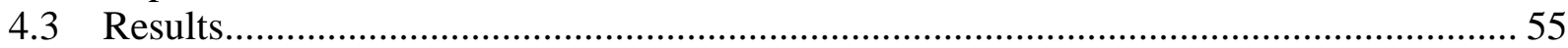

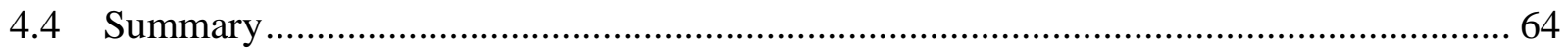

CHAPTER 5: WAVELENGTH AND POLARIZATION DEPENDANCE OF TIP-ENHANCED

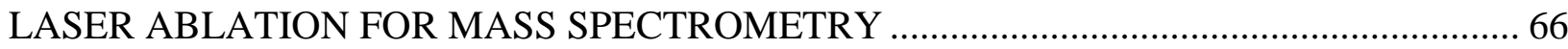

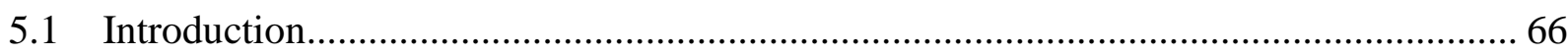

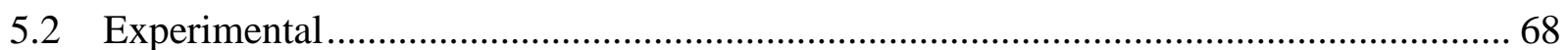

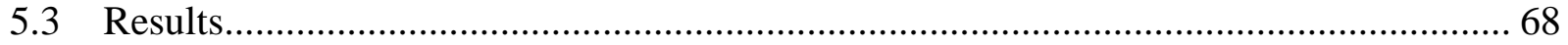

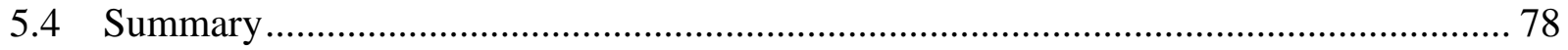




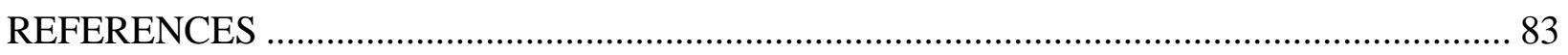

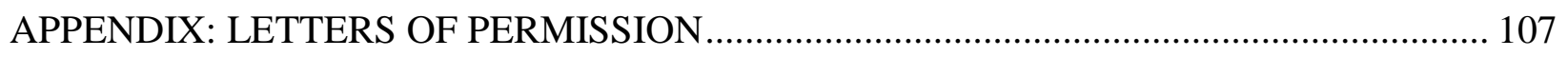

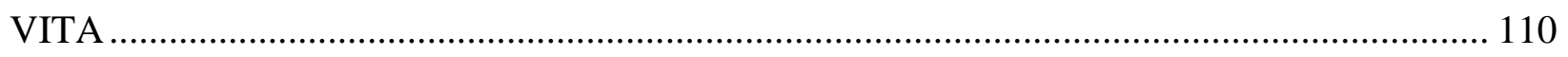




\section{LIST OF FIGURES}

Figure 1-1 Operating principle of AFM......................................... 20

Figure 1-2 AFM cantilever motion in tapping mode................................... 21

Figure 1-3 Aperture and apertureless near-field modes............................ 22

Figure 1-4 A schematic of aperture mode near-field laser ablation mass

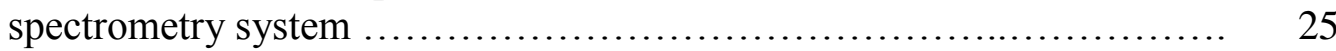

Figure 1-5 Apertureless near-field laser ablation ICP-MS system ................... 26

Figure 1-6 Schematic of combined AFM and LDI-MS imaging system ............. 27

Figure 2-1 Photograph of the AFM tip-enhanced laser ablation system ............... 31

Figure 2-2 Photograph of the sampling stage and tip of afm+ instrument .............. 32

Figure 2-3 SEM images of the AFM probe (a) before use and (b) damaged after exposure to 1000 laser shots ...................................... 33

Figure 2-4 Schematic of the AFM tip-enhanced laser ablation system .................. 34

Figure 2-5 Mounted silver wire positioned $300 \mu \mathrm{m}$ above the AFM tip ................. 35

Figure 3-1 Near-field laser produced craters using AFM. (a) and (b) show AFM height images before and after the ablation experiment respectively with anthracene thin film; whereas the height images for ablation of rhodamine $6 \mathrm{G}$ are shown in (d) and (e). The depth profiles of the crater are shown in (c) and (f) for anthracene and rhodamine 6G respectively........................................................

Figure 3-2 LDI mass spectra of (a) blank silver wire, (b) silver wire dipped in anthracene solution and (c) near-field laser ablation capture on silver wire.

Figure 3-3 LDI mass spectra of (a) silver wire dipped in rhodamine 6G solution and (b) near-field laser ablation transfer from 18 ablation spots to a silver wire.

Figure 3-4 Tip-enhanced laser assisted sample transfer of the peptide angiotensin II showing a) AFM image of a laser produced crater and b) MALDI mass spectrum of material transferred from a single spot 
Figure 3-5 MALDI mass spectrum of bovine insulin transferred from 10 spots using tip-enhanced laser assisted sample transfer ..........................

Figure 4-1 AFM images of (a) $5 \mu \mathrm{m}$ x $5 \mu \mathrm{m}$ region of a deposited peptide mixture; the light region indicated by the green arrow corresponds to the sample; (b) material removal is observed after ablation from the top half of the sample and the green arrow indicates the material targeted for ablation; (c) removal of some of the remaining material; (d) sample region after complete material removal............................................

Figure 4-2 Mass spectrum of a peptide mixture ablated and captured for MALDI analysis

Figure 4-3 Insulin ablation crater from $532 \mathrm{~nm}$ tip-enhanced laser ablation.

Figure 4-4 MALDI mass spectrum of the protein insulin using AFM tip-enhanced ablation and capture...

Figure 4-5 TELA produced crater on rat brain tissue using AFM. (a) and (b) show AFM height images before and after the ablation experiment respectively with rat brain tissue section. The depth profile of the crater is shown in (c) for rat brain tissue section

Figure 4-6 Off-line MALDI mass spectra from rat brain tissue sample (a) solvent extracted rat brain tissue sample (b) from TELA produced crater shown in Figure 1 b. A magnified view of the $m / z$ 600-1100 region is shown in the inset for each spectrum. In this spectrum, prominent ions at $\mathrm{m} / \mathrm{z} 734.6$ $[\mathrm{PC}(32: 0)+\mathrm{H}]^{+}, 760.6[\mathrm{PC}(34: 1)+\mathrm{H}]^{+}$, and $788.6[\mathrm{PC}(36: 1)+\mathrm{H}]^{+}$are shown. PC indicates phosphatidylcholine, and the numbers in parentheses indicate the alkyl chain length and number of double bonds, respectively

Figure 4-7 Off-line MALDI mass spectra from two different areas (a) capture spot 1 and (b) capture spot 2, on rat brain tissue sample captured using nearfield laser ablation sample transfer...

Figure 5-1 AFM images of ablation crater at different polarization angles..............

Figure 5-2 Ablation crater volume plotted as a function of rotation of polarization of the laser. $0^{\circ}$ and $180^{\circ}$ represents p-polarization, whereas $90^{\circ}$ is spolarization in the plot 
Figure 5-3 AFM-TELA at different wavelengths (a) ablation crater with a single shot of $532 \mathrm{~nm}$ laser wavelength at $850 \mathrm{~J} / \mathrm{m}^{2}$ fluence, (b) example of ablation craters with 30 shots of $355 \mathrm{~nm}$ laser at $850 \mathrm{~J} / \mathrm{m}^{2}$ fluence. (c) Comparison of depth profile of the craters created with $355 \mathrm{~nm}$ and 532 $\mathrm{nm}$ laser wavelength.

Figure 5-4 Volume of the ablation crater as a function of laser fluence at $532 \mathrm{~nm}$

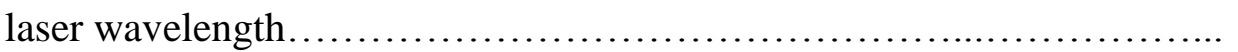

Figure 5-5 Volume of the ablation crater as a function of laser fluence at $1064 \mathrm{~nm}$

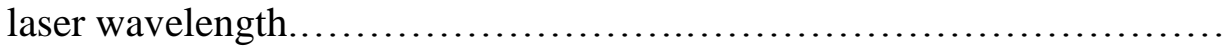

Figure 5-6 Volume of material ablated is plotted as a function of number of laser shots at different laser fluences

Figure 5-7 Material deposited around the ablation craters (a) First AFM scan after ablation showing melting on the perimeter of the crater. (b) Same crater has been scanned for 5 times and the amount of amount of melted materials decreased considerably, indicating very low density of the melted material

Figure 6-1 Gel electrophoresis of PCR products of TELA captured DNA plasmids.... 


\section{LIST OF ABBREVIATIONS AND SYMBOLS}

AFM

AP

APCI

CE

CHCA

CHCA

DHB

EI

ESI

FTICR

ICP

LA

LAMMA

LDI

MALDI

MS

MSI

$\mathrm{Nd}: \mathrm{YAG}$

NF

SERS

SIMS

SMALDI
Atomic force microscopy

Atmospheric pressure

Atmospheric pressure chemical ionization

Capillary electrophoresis

a-cyano-4-hydroxycinnamic acid

a-cyano-4-hydroxycinnamic acid

2,5-dihydrobenoic acid

Electron ionization

Electrospray ionization

Fourier transform ion cyclotron resonance

Inductively coupled plasma

Laser ablation

Laser microprobe mass analyzer

Laser desorption ionization

Matrix assisted laser desorption/ ionization

Mass spectrometry

Mass spectrometry imaging

Neodymium-doped yttrium aluminum garnet

Near-field

Surface enhanced Raman spectroscopy

Secondary ion mass spectrometry

Scanning microprobe matrix assisted laser desorption/ ionization 
SNOM Scanning near-field optical microscopy

TEF Tip enhancement factor

TELA Tip-enhanced laser ablation

TEM Transmission electron microscopy

TOF Time of flight 


\begin{abstract}
In this research, atomic force microscope tip-enhanced laser ablation mass spectrometry (AFM TELA-MS), an ambient sub-micrometer scale sampling method for offline MS was developed. AFM TELA was used to transfer molecules from thin films to a suspended silver wire for off-line mass spectrometry using laser desorption ionization (LDI) and matrix-assisted laser desorption ionization (MALDI). An AFM with a $30 \mathrm{~nm}$ radius gold-coated silicon tip was used to image the sample and to hold the tip $15 \mathrm{~nm}$ from the surface for material removal using a pulsed Nd:YAG laser, which provides output at wavelengths of $532 \mathrm{~nm}$ in the visible, $1064 \mathrm{~nm}$ in the near IR, or the $355 \mathrm{~nm}$ UV wavelength. The laser is mildly focused onto the AFM tip and the fluence is set just below the far-field ablation threshold to irradiate the AFM tip for material removal with a smaller spot size than a laser focused with a conventional lens system. The AFM is used to image ablation craters and place the tip at the area being analyzed. For small molecules, approximately $100 \mathrm{fg}$ of material was ablated from each of the $1 \mu \mathrm{m}$ ablation spots and transferred with approximately 3\% efficiency. AFM-TELA of large biomolecules was also demonstrated at $3 \%$ efficiency and a mass range up to 600 Da. AFM-TELA studies with different laser parameters indicated that the tip-enhanced material ejection depends on laser wavelength, polarization, fluence, and number of laser shots used for material ejection, but not on the absorption of the sample itself. The utility of AFM-TELA was applied to sampling of rat brain tissue. The ability of producing sub-micrometer scale craters, capture on a suspended silver wire and detection of lipids were demonstrated using off-line MALDI MS.
\end{abstract}




\section{CHAPTER 1. INTRODUCTION}

The work discussed in this dissertation is aimed at exploring the utility of atomic force microscope (AFM) assisted tip-enhanced laser ablation (TELA) sample transfer for biomolecule mass spectrometry. Hence, the significance of acquiring spatially resolved chemical information and the role of mass spectrometry as a suitable candidate for acquiring spatially resolved chemical information are discussed. After a more general introduction to scientific background of mass spectrometry and laser ablation and desorption, this chapter covers the use of laser ablation and desorption for mass spectrometry imaging of biomolecules at high lateral resolution. This chapter also contains an overview of near-field optics and atomic force microscopy as they relate to this research.

\subsection{Mass Spectrometry Sampling and Imaging}

Mass spectrometry is a technique that can be used selectively to analyze chemical compounds in analytical chemistry: a mass spectrometer determines the mass of atomic and molecular ions by measuring the mass to charge ratio $(m / z) \cdot{ }^{1-3}$ Since mass spectrometry is based on measuring the mass to charge ratio of the analyte, the first step in MS analysis, ionization, is critical. There are many ionization techniques have been developed depending on the type of molecule and the degree of fragmentation desired. ${ }^{4}$ Specifically, biomolecules are not easily transferred into the gas phase and ionized. In order to analyze large biomolecules, a "soft" ionization technique is desired to prevent fragmentation of molecules during ionization. Matrix assisted laser desorption/ionization (MALDI) and electrospray ionization (ESI) are the most widely used soft ionization methods for analyzing intact large biomolecules such as proteins, peptides, and lipids. ${ }^{5-7}$ Development of these methods was the basis for the increasingly powerful instrumentation that have become available for biomolecule analysis. Furthermore, the 
introduction of software algorithms in early 1990's has led to an exponential increase in the number of publications using MS for the biomolecules analysis and proteomics. ${ }^{8-9}$ These algorithms allowed the correlation of MS data with protein sequence databases. The rapid development of instrumentation, advances made in sample preparation for $\mathrm{MS}$, and the availability of sequence databases have enabled the transformation of mass spectrometry from small molecule analysis to large scale biomolecule analysis. ${ }^{10-11}$

Mass spectrometry imaging (MSI) is a technique which involves acquiring an array of mass spectra at a regular intervals in a spatially defined area of an object, then choosing the desired peaks in the resulting spectra to generate a heat map image that correlates the regions on the sample surface with the chemical species. ${ }^{12-13}$ To obtain a mass spectral image, samples under vacuum are bombarded by an energetic beam of ions or irradiated with a pulsed laser. Under ambient conditions, pulsed lasers can be used and, in addition, charged particles and ions can be used to generate ions. Compounds can also be extracted by a solvent in contact with the surface or laser ablated, captured, and extracted. ${ }^{14-15}$

The chemical specificity and versatility inherent to MS has led MSI to become a powerful chemical imaging tool. Hence, MSI has been applied extensively in forensics, microbiology, biomedical researches and in pharmaceutical sciences. ${ }^{16-18}$ Typically in MSI, sampling can be accomplished in microprobe mode ${ }^{19-20}$ or in microscope mode. ${ }^{21}$ Microprobe mode, the more widely used method, involves scanning a focused laser or ion beam to desorb material from the sample surface, or a sampling inlet that extracts compounds from the sample, across a localized sample surface. Mass analyzers and detectors are then used to determine the masses of the analytes. The resulting mass spectra are stored along with the spatial coordinates of the sample and the ion images are then reconstructed from the individual mass spectra. ${ }^{15}$ 
In microscope imaging mode, the desired area is sampled at once and ions are directed to a position sensitive detector via a mass analyzer, which records spatial information. ${ }^{22}$ Furthermore, this technique uses an unfocused primary laser or ion beam to irradiate the sample surface, typically much larger area of a sample compared to laser based microprobe methods. ${ }^{23}$ Desorbed ions are then directed with a time of flight mass analyzer that maintains the spatial orientation of the ions. Although, microscope mode enables higher lateral resolution to be achieved even without a focused beam, obtaining higher mass accuracy, resolution and performing tandem MS is problematic. ${ }^{24}$

The most compelling advantage of imaging mass spectrometry is the capability of imaging biological specimens at high mass resolution and obtaining chemical information for the compounds in biological specimens without any aid of staining or chemical tagging. ${ }^{25-27}$

Techniques such as secondary ion mass spectrometry (SIMS) $)^{28-31}$ and MALDI $^{32-33}$ are commonly used for surface sampling and chemical imaging. With SIMS, a focused high-energy primary ion beam ablates and ionizes material from the sample. The resulting secondary ions are sent to a quadrupole, sector, or time-of-flight (TOF) MS for analysis. The sensitivity and mass range of SIMS have been extended with TOF mass analyzers ${ }^{34}$ and cluster ion sources. ${ }^{35-36}$ Recent reports on SIMS imaging have shown the capability of imaging atoms and small molecules down to $35 \mathrm{~nm}$ lateral resolution ${ }^{37}$ and molecules up to approximately $1500 \mathrm{Da}$ at a spatial resolution of a few hundred nanometers. ${ }^{36}$ Excellent lateral resolution is associated with SIMS that allows analysis of biomolecules at the sub-cellular level lateral resolution, but the main drawback is the fact that it is a high fragmentation, "hard" ionization method and therefore limited to elemental chemical information and low mass species. ${ }^{30,38}$ 


\subsubsection{SIMS Imaging}

Among the other MSI methods, SIMS is a surface sensitive technique, which allows acquisition of chemical information from the top few layers of a sample. ${ }^{31,39-40}$ Primary ion beams used in SIMS instruments, typically with a kinetic energy in the $\mathrm{kV}$ range, enables collecting sub-micron size pixels from the sample surface. However, the useful ion yield from pixels of that size range is limited due to the poor percentage of sputtered molecules from the sample surface. ${ }^{31,41}$ The energetic desorption process associated with SIMS yields significant fragmentation of desorbed material. ${ }^{42}$ Hence, SIMS has been most successful for inorganic molecules, low molecular weight biomolecules $(<1500 \mathrm{Da})$, or simply to acquire elemental distributions. ${ }^{43-44}$ Moreover, a typical SIMS experiment does not require addition of a matrix. Therefore, SIMS analysis yields higher spatial resolution especially in the low mass region. ${ }^{30}$

There are two operational modes in SIMS: static SIMS and dynamic SIMS. ${ }^{45}$ The difference is made based on the primary beam fluence related and the surface damage $\left(>10^{13}\right.$ ions $/ \mathrm{cm}^{2}$ for dynamic SIMS). Dynamic SIMS instruments are more suitable for subcellular studies of elemental distributions and the localization of targeted small molecules. Static SIMS with primary beam fluencies below $10^{13}$ ions $/ \mathrm{cm}^{2}$ is predominantly employed for the analysis of larger organic molecules and tissue analysis. ${ }^{44}$ The introduction of cluster ion sources and TOF mass analyzers has significantly improved the formation of secondary ions allowing sampling of small peptides and oligonucleotides from biological samples. ${ }^{28,46}$ When delivering primary ion clusters such as $\mathrm{Au}^{3+}, \mathrm{Bi}^{3+}$, or $\mathrm{Bi}^{5+}$ on biological samples, significantly enhanced intensities were observed. This was mainly due to much higher sputter yield. ${ }^{47}$ The use of cluster ion beams for sample bombardment is gentler and enhances the yield of higher mass species. ${ }^{43}$ Polyatomic ion clusters can be used to obtain ion images with high sensitivity and micrometer scale lateral 
resolution up to $1500 \mathrm{~m} / \mathrm{z}^{44}$ Biological imaging using SIMS is an expanding area of research and many SIMS based biological imaging applications have been recently reviewed. ${ }^{16,24,30,48-49}$

\subsubsection{Laser Desorption Ionization Imaging}

\section{Laser Microprobe Mass Spectrometers}

The applications of laser desorption and ablation have been well established in different areas including chemical analysis, ${ }^{50-51}$ surgeries, ${ }^{52-53}$ micromachining, ${ }^{54-55}$ and pulsed laser deposition. ${ }^{56}$ In general, laser desorption can be defined as laser induced thermal evaporation of molecules from the outer layers of the targeted sample. ${ }^{57-59}$ Laser ablation, occurs when the laser energy is rapidly transferred on to the sample to produce a massive ejection of material in the form of clusters and particles. ${ }^{60}$ There are many advantages of laser ablation sampling when compared with conventional sample dissolution methods. These advantages include minimal sample preparation, ability to perform ambient ablation for sampling, and allow obtaining localized chemical information of the sample with a focused laser beam.

The concept of localized chemical analysis of organic and biological samples was first developed in 1975 with laser microprobe mass analysis. ${ }^{61-63}$ In laser microprobe analysis, the spatial distribution of analytes are investigated by moving the target in an $x-y$ direction in raster mode under a focused laser while simultaneously collecting and mass analyzing representative ions. Early laser microprobe experiments performed by Hillenkamp and coworkers obtained approximately $1 \mu \mathrm{m}$ resolution by focusing ruby laser $(\lambda=347 \mathrm{~nm})$ to irradiate the sample and the desorbed ions were directed into time of flight mass spectrometer for analysis. ${ }^{61-62}$ Although, this technique allowed $1 \mu \mathrm{m}$ lateral resolution, samples must be kept in vacuum in order to be analyzed by the time of flight mass spectrometry (TOF-MS). As a solution for this limitation, the first ambient LDI method in laser microprobe mode was introduced by Holm et al. ${ }^{64}$ In ambient 
laser microprobe LDI, $0.2 \mu \mathrm{m}$ thick sample sections were kept under atmospheric pressure conditions on a LAMMA 500 instrument and ablated with a pulsed focused laser at $265 \mathrm{~nm}$ wavelength to generate approximately $2 \mu \mathrm{m}$ holes on the sample in transmission geometry. LDI generated ions were then analyzed by a TOF MS. However, using conventional laser optics, obtaining higher lateral resolution was limited.

Lateral resolution in laser mass spectrometry imaging is determined by a number of factors. According to the Abbe equation, the lateral resolution of an optical microscope is limited by diffraction. ${ }^{65}$ The lateral resolution $\Delta x$, of standard optical microscopy is given by the Equation 1.1. ${ }^{66-68}$

$$
\Delta x=\frac{0.61 \lambda}{N A}
$$

Where, $\lambda$ is the wavelength of the interacting radiation, and $N A=n \sin \alpha$, is the numerical aperture of the objective lens. For better lateral resolution, the $N A$ can be increased by a large index of refraction $n$ of the surrounding medium or a large half-angle $\alpha$. However, there are practical limitations achieving higher NA for better lateral resolution. High numerical aperture lenses tend to be wide and must be positioned close to the sample with high index of refraction immersion oil, properties that are not suited to laser desorption and ablation mass spectrometry. Thus the focused laser diameter used for mass spectrometry imaging is often far from the diffraction limit. Other factors affecting imaging resolution are the raster step size and resulting image pixel size, which is typically in the same range as the laser diameter.

The diffraction limited spot size $\left(D_{f}\right)$ of a laser beam is given by Equation 1.2. ${ }^{69-71}$

$$
D_{f}=M^{2} \cdot \frac{4}{\pi} \cdot \lambda \cdot \frac{f}{D_{0}}
$$


where $\lambda$ is the wavelength of laser, $f$ is the focal length of focusing lens, $D_{0}$ is the input beam diameter, and $\mathrm{M}^{2}$ is the beam quality factor $\left(\mathrm{M}^{2}=1\right.$ for an ideal Gaussian beam profile) Therefore, optimizing the beam shape (minimizing $\mathrm{M}^{2}$ ) and minimizing the $f / D_{0}$ ratio, (by placing the focusing lens as close to the sample as possible and expanding the laser before the focusing lens) will minimize the laser spot size $\left(D_{f}\right)$.

Because of its versatile features, laser microprobe mass spectrometry found broad applications over the years and various areas of application of the laser microprobe have been reviewed in several papers. ${ }^{72-73}$ Although the resolution of the laser microprobe is limited by the laser focus, it provides excellent elemental and small molecule analysis capabilities and opened multiple avenues to performing LDI MS of biological samples. ${ }^{74-75}$

Several other approaches for high lateral resolution MSI have also been demonstrated using reflective mirror focusing objectives such as Schwarzschild type optics. ${ }^{76-77}$ Behm et al. utilized internal laser desorption coupled with laser post-ionization. ${ }^{78}$ The laser beam was steered, to enable line scan imaging, into a Schwarzschild microscope objective via a mirror on a motor driven mount placed before the objective to focus the desorption laser beam. The unique arrangement of the two concentric spherical mirrors in the Schwarzschild microscope eliminated the beam aberrations and made it inherently achromatic. A Schwarzschild objective was used for laser desorption with $1 \mu \mathrm{m}$ lateral resolution with a Fourier transform ion cyclotron resonance (FTICR) mass spectrometer. ${ }^{78}$ The system used a $337 \mathrm{~nm}$ nitrogen laser for desorption and, for some cases, the fifth harmonic of a Nd:YAG laser at $213 \mathrm{~nm}$ was used for post-ionization. The nitrogen laser was focused with an objective that was $11 \mathrm{~cm}$ in diameter and $18 \mathrm{~cm}$ in length and had a numerical aperture of 0.2 and a $13 \mathrm{~cm}$ working distance. The desorption laser was focused onto the sample target that was placed within the FTICR magnet bore and adjacent to the ICR 
cell. The post-ionization laser was directed above the target using a set of steering prisms. The lateral resolution in line scan mode for direct laser desorption ionization of $\mathrm{Cu}^{+}$ions from a copper grid and laser desorption and post ionization of a $\mathrm{C}_{60}$ thin film on indium was less than 1 $\mu \mathrm{m}$. Even though, a sub-micrometer lateral resolution can be achieved, Schwarzschild type optics have shown only limited applications for MS imaging.

\section{MALDI Imaging}

In addition to the ion collection efficiency, the resolution of MALDI imaging is influenced by laser optics as well as sample preparation steps. ${ }^{20}$ In MALDI, a light absorbing matrix must be added, which serves as an energy absorber and promotes the formation of biomolecules without fragmentation. Two decades ago, Caprioli and coworkers introduced MALDI imaging in reflectron geometry using a commercial MALDI TOF instrument with a lateral resolution of $25 \mu \mathrm{m} .{ }^{13}$ Over the years, several improvements in signal digitization electronics and the replacement of relatively low repetition rate desorption lasers with faster repetition rates have enabled faster data acquisition in MALDI instruments. ${ }^{79}$ However, the lateral resolution of commercial MALDI instruments has not changed significantly and it is still remains within $25-10 \mu \mathrm{m}$ range. ${ }^{80}$

There are several factors to be considered when improving the lateral resolution of MALDI imaging: (1) matrix addition, (2) oversampling, (3) improving beam focus, and (4) transmission geometry. In MALDI, the detection of analyte molecules depends on the matrix used and its interaction with the sample. However, matrix addition can result in the delocalization of sample components. ${ }^{15,81}$ Several procedures have been introduced to reduce analyte migration. For example, MALDI imaging using dry matrix addition enabled obtaining images at a resolution of $10 \mu \mathrm{m}$. The matrix is sublimed in a vacuum apparatus to achieve a 
coverage of approximately $0.2 \mathrm{mg} / \mathrm{cm}^{2}$ at a thickness of $2 \mu \mathrm{m} .{ }^{82} \mathrm{Next}$, the incubation of sublimed matrix and tissue with the solvent with low heat, allowed extraction of tissue components and matrix recrystallization with smaller than $3 \mu \mathrm{m}$ in diameter. Metal sputter coating is an alternative technique for dry matrix application. A recent study has shown that small molecules can be detected and imaged with a lateral resolution of $5 \mu \mathrm{m}$, when thin film of silver was sputter coated on tissue sections. $^{83}$

Another approach for improving the lateral resolution of MALDI imaging is oversampling, where, features considerably smaller than the irradiating laser beam are imaged. In this technique, no modifications to the MALDI instrument are required to achieve lateral resolution less than the laser diameter. This is done by adjusting the imaging step size to a value lower than the laser spot diameter and the sample is irradiated until no further signal is obtained. ${ }^{84-85}$ Although, higher lateral resolution is achieved, longer data acquisition and loss of signal are some of the drawbacks associated with oversampling. ${ }^{79}$

According to the Equation 1.2, the beam quality can be improved if the laser is sent through an optical fiber. ${ }^{86-87}$ However, sending high energy pulses through optical fibers are problematic. Therefore, this approach is not ideally suited for delivering a laser in MALDI systems. In contrast, higher lateral resolution in MALDI MSI can be achieved by modifying the laser optics of a MALDI-TOF mass spectrometer. Recently, the laser optics of a commercially available MALDI TOF/TOF mass spectrometer was modified to obtain a Gaussian beam in combination with an aspheric lens to improve the lateral resolution to $5 \mu \mathrm{m} .^{69,88}$ The diffraction limited focusing of a Gaussian laser beam on the target was achieved by beam expansion combined with filtering of non-Gaussian beam profile components using a pinhole to obtain a Gaussian beam profile. Several other approaches have revealed improving beam quality factor 
and minimizing $f / D_{0}$ ratio (see Equation. 1.2) to increase the lateral resolution in MALDI MSI and have been demonstrated for various tissue types and single cells. ${ }^{89-90}$

In addition to the modifications that have been applied to MALDI MSI for submicrometer lateral resolution, Caprioli and co-workers demonstrated the capability of MSI at sub-cellular lateral resolution and at high acquisition speed by integrating a transmission geometry ion source with TOF-MS. ${ }^{91-92}$ Imaging in transmission geometry enabled a larger numerical aperture lens to be used. The samples were irradiated using a laser beam (1-2 $\mu \mathrm{m}$ laser spot size) focused from the back side of the sample placed on a glass slide, they demonstrated sub-micrometer level MSI for a few targeted immune-labeled antibodies as well as for tissue proteins with a $1 \mu \mathrm{m}$ laser spot diameter and $2.5 \mu \mathrm{m}$ raster step size. ${ }^{93}$ Although, the lateral resolution was improved, there are some practical limitations associated with this method: (1) a fundamental reconfiguration to the MALDI imaging instrument is needed and (2) successful laser penetration requires thin tissue sections.

All of the MSI methods described so far utilize microprobe sampling for imaging. An alternative approach is performing MSI in microscope mode, where ions are created simultaneously across an area of the sample and the detection is performed maintaining their spatial orientation. Heeren and co-workers have developed a laser ionization mass microscope, suitable for high lateral resolution MS imaging of large biological molecules such as peptides and proteins. $^{21-22,94}$ The attainable lateral resolution is independent from the size of the desorption/ionization laser. They obtained mass resolved images of intact peptides and proteins by irradiating an area of $200 \mu \mathrm{m}$ while retaining the ion spatial distribution during TOF followed by ion detection with a position sensitive detector with a lateral resolution of $4 \mu \mathrm{m}$ in less than 1 ms using both IR and UV lasers. ${ }^{21,94}$ The decoupling of lateral resolution from the spot size of 
the focused laser beam enabled analyzing larger sample area even without moving either sample or the laser, but this method can only be applied in a vacuum. ${ }^{95}$

A MALDI ion source with a high numerical aperture through-hole objective has been

developed. ${ }^{96-97}$ In this work, high lateral resolution was achieved by directing the ions through a hole drilled in the objective. In early experiments, sub-micrometer lateral resolution was achieved by two step laser focusing: first the laser was pre-focused to $10 \mu \mathrm{m}$ outside the vacuum and the final focusing was performed to obtain $0.5 \mu \mathrm{m}$ laser spot for LDI or MALDI analysis. ${ }^{96}$ $100 \times 100 \mu \mathrm{m}$ chemical images were obtained with a 50 pixels per second scan rate with 0.6 to $1.5 \mu \mathrm{m}$ lateral resolution for biological samples with the combination of MALDI imaging and confocal microscopy. Further advancements of the technique made the system suitable for ambient analysis and enabled high lateral resolution imaging for mass spectrometry at high mass accuracy by coupling with ion trap Fourier transform ion cyclotron resonance (FTICR) $\mathrm{MS}^{98}$ Recently, the technique was utilized for imaging of human renal carcinoma cells with $2 \mu \mathrm{m}$ lateral resolution. ${ }^{99}$

\subsubsection{Ambient Sampling and Imaging Mass Spectrometry}

All of the MSI methods described to this point have the drawback that analysis must be carried out in vacuum, which limits the type of the sample that can be analyzed due to the constraints of placing the sample into the vacuum. ${ }^{20,100}$ Imaging of biological systems at ambient conditions is an emerging field because preparation of samples for analysis in vacuum is difficult and time consuming. Ambient mass spectrometry is the direct analysis of materials in their native environment with ions created outside the mass spectrometer. ${ }^{101-104}$ In ambient mass spectrometry, the sample can be bombarded by charged droplets, ions, metastable atoms, or laser irradiated and material is removed by extraction, heating, laser ablation, or acoustic shock. The 
most compelling advantage of ambient mass spectrometry comprises capability of coupling highly localized sample desorption techniques with mass spectrometry to perform subcellular level MS sampling at atmospheric pressure. The rapid development in the number of ambient sampling and ionization techniques has led to an increase in the number of publications utilizing MSI for the detection of biomolecules. ${ }^{105-108}$ Desorption electrospray ionization (DESI) and direct analysis in real time (DART) are the leading methods for ambient ionization, which form ions at the sample surface by charged droplets or ions respectively. ${ }^{12,109}$

\section{Desorption Electrospray Ionization}

The introduction of MS sampling under ambient conditions with DESI in 2004 opened up several avenues for the development in ambient imaging mass spectrometry. ${ }^{110-111}$ In DESI, highly charged microdroplets generated by ESI impinge on the sample surface to extract the analyte molecules. The subsequent secondary droplets are removed from the sample surface and sampled into the inlet of the mass spectrometer. DESI-MSI is performed by desorbing the analytes with a stationary continuous flux of charged droplets, while rastering the sample surface. Mass spectra containing $\mathrm{m} / \mathrm{z}$ and relative abundance information are then collected at each position to construct the spatial distribution of intensity of a specific $\mathrm{m} / \mathrm{z}$ value. ${ }^{112}$

Chemical profiling of biological tissue samples using DESI MS was first reported in 2005. ${ }^{113}$ Animal tissue sections were analyzed using DESI without any sample pretreatment and the variation of phospholipid profiles over a tumor tissue was demonstrated. The capability of utilizing DESI for MSI of small molecules such as lipids has also been demonstrated. ${ }^{114}$ Two dimensional MS imaging has been demonstrated by employing two motion stages with DESI ion sources. Furthermore, several studies have demonstrated the utility for distinguishing between healthy human tissues and diseased tissues. ${ }^{115}$ The lateral resolution of DESI MSI is typically 
around a few hundred micrometers; however, a lateral resolution of $35 \mu \mathrm{m}$ was reported for mouse cerebellum tissue sections. ${ }^{116}$

Improving the lateral resolution of DESI MSI has concentrated on several factors such as the diameter of the emitter capillary, emitter incident angle, spray tip-surface distance, solvent composition, nebulizing gas flow rate, and step-size of the scan. ${ }^{116}$ There have been a number of attempts and documented procedures to maximize resolution in DESI MSI; however, none of these has yet achieved resolution below $35 \mu \mathrm{m}$ range for imaging of large molecules from biological specimens. ${ }^{39,117}$

\section{Extraction Methods}

In addition to the conventional MSI methods, direct liquid extraction methods for tissue and single cell analysis have also emerged in the last decade. Progress in improving lateral resolution has been made with the introduction of the surface sampling probe (SSP) method nanospray desorption ionization (nanoDESI). ${ }^{118-119}$ In nanoDESI, molecules are extracted into a liquid microjunction formed between two capillaries at the sample surface. One capillary continuously supplies solvent to create and maintain the liquid microjunction, while the second capillary electrosprays the extracted molecules to form ions that are sampled by the mass spectrometer. ${ }^{120-122}$ Studies have shown that nanoDESI imaging can be used to characterize lipids on biological samples, allowing high lateral resolution approximately around $10 \mu \mathrm{m}$ on biological samples. ${ }^{118,123-124}$ The "single probe" microextraction approach, which is similar to nano-DESI concept, has also been demonstrated for sampling of cells and tissue. ${ }^{123}$ The cellular material is extracted into a nanospray solvent at the liquid junction of the tissue and the sample probe and extracted material are detected using nano ESI MS. ${ }^{123,125}$ Mass spectrometry imaging of larger molecules such as proteins and peptides are also possible with nanoDESI. However, 
such approaches have not yet achieved resolution below $100 \mu \mathrm{m} .{ }^{126}$ A recent study has shown the capability of imaging proteins up to $15 \mathrm{kDa}$ in tissue samples using nanoDESI with a lateral resolution of $200 \mu \mathrm{m} .^{127}$

Direct micro sampling ESI has been used to explore the chemical heterogeneity in tissue samples and single cells. ${ }^{128}$ Extraction methods can be used for sampling of biological material and have the advantage that the sampled material can be further processed prior to ionization and MS detection. ${ }^{129}$ Probe ESI (PESI) has been demonstrated for sampling material from plant cells using different surfaces such as, modified glass rods, metal tips etc. ${ }^{130}$

\section{Discharge Methods}

DART mass spectrometry allows analysis of low-molecular mass volatile compounds by bombarding the sample with metastable ions from a glow discharge to form ions at ambient conditions. ${ }^{131-133}$ DART-MS has been used for analysis in various fields such as, pesticide monitoring on vegetables, screening for traces of explosives, and forensic applications. ${ }^{134-135}$

There are several other plasma based techniques have been developed, ${ }^{136}$ allowing ambient sampling for MS such as, dielectric barrier discharge ionization (DBDI), ${ }^{137}$ plasma-assisted desorption/ionization (PADI), ${ }^{138}$ atmospheric solids analysis probe (ASAP), ${ }^{139}$ and the flowing atmospheric-pressure afterglow (FAPA). ${ }^{140}$ Although, plasma based ambient sources have some advantages over ESI and MALDI, these ionization sources typically produce streams that are several millimeters in diameter. ${ }^{132,136}$ Therefore, the use of plasma-based ambient ionization sources for MSI is limited and has not been explored in detail. ${ }^{140}$

\section{Laser Ablation Ambient Mass Spectrometry}

There are several laser-based approaches have been developed for ambient mass

spectrometry. ${ }^{103-104,108,141}$ In these techniques, lasers are used to remove material by desorption 
or ablation and either directly ionize or deliver it to a secondary ionization source such as ESI or metastable cluster ion. ${ }^{101,105,142-144}$

Atmospheric pressure MALDI (AP-MALDI) is laser based ionization technique, where the ions are created directly during the laser/material interaction. AP- MALDI with an IR laser and endogenous matrix ${ }^{145-147}$ and femtosecond pulse width laser matrix-free ionization ${ }^{148}$ have been reported. However, the sensitivity in AP-MALDI can be limited due to the inefficient delivery of ions into the mass spectrometer. ${ }^{101}$

Lasers can be used for ambient ionization in a two-step process involving the initial desorption or ablation of neutral molecules or particles followed by post ionization. There are various types of post-ionization that have been developed. Each technique differs from the others in terms of its sampling and ionization, allowing analysis of compounds in complex samples. Post-ionization techniques are based on inductively coupled plasma, ${ }^{149-151}$ chemical ionization, ${ }^{152-153}$ electrospray ionization, ${ }^{142,148,154-155}$ and photoionization, ${ }^{156}$ typically employed with lasers that are focused to spot sizes around $100 \mu \mathrm{m}$ in diameter.

The most compelling advantage of laser-based approaches is ambient imaging. Several reports on laser ambient imaging mass spectrometry ${ }^{157}$ have shown feasibility of ambient imaging of small molecules at $20 \mu \mathrm{m}$ resolution with infrared laser ablation metastable -induced chemical ionization (IR-LAMICI) ${ }^{158}$ and imaging of larger molecules such as peptides and lipids at $40 \mu \mathrm{m}$ resolution using IR lasers with MALDI-MS. ${ }^{107,147}$ Ambient mass spectrometry imaging by laser ablation electrospray ionization (LAESI) has also been accomplished to determine the spatial distribution of bio molecules in a wide variety of biological samples. ${ }^{159-162}$ Furthermore, a cell-by-cell imaging protocol for a monolayer of single cells using a LAESI system was demonstrated by Li et al. ${ }^{163}$ In this approach, an etched optical fiber transmitting mid-IR light is 
brought in close contact to individual cells of interest to perform localized ablation. Matrixassisted laser desorption electrospray ionization (MALDESI) is an ambient ionization method that combines attributes from both MALDI and ESI. ${ }^{164}$ Here, an ultraviolet (UV) or infrared (IR) laser can be utilized to excite the matrix, that facilitates the desorption of neutral molecules from the sample by absorbing the energy of the laser. The ejected molecules are then extracted into the charged droplets of electrospray and are sampled by the mass spectrometer. ${ }^{165}$ The utility of MALDESI for high lateral resolution MSI has been explored for vast range of applications. ${ }^{166-169}$

Another approach for high lateral resolution ambient MALDI MSI was reported by Spengler and co-workers, employing the coaxial objective in reflection mode and the ionization source was adapted allowing ambient MALDI imaging of biomolecules. ${ }^{98}$ Using this high numerical aperture coaxial objective, a $337 \mathrm{~nm}$ nitrogen laser was focused to a spot size of 0.5 $\mu \mathrm{m} \times 0.6 \mu \mathrm{m}$. Imaging of peptide standards was achieved with a laser diameter of $8 \mu \mathrm{m}$ due to sensitivity limitations with smaller spot sizes. Lateral resolution of $5 \mu \mathrm{m}$ was also reported with an atmospheric MALDI imaging system coupled with an Orbitrap mass spectrometer. ${ }^{170}$ A 337 $\mathrm{nm}$ nitrogen laser was focused to a $8 \mu \mathrm{m}$ spot using a high numerical aperture reflection geometry configuration. Images of neuropeptides in mouse pituitary were imaged at $5 \mu \mathrm{m}$ lateral resolution. The instrument has also been used to image lipids in whole body sections of mosquitos. $^{171}$

Coupling laser ablation with a liquid microjunction surface sampling probe (LMJ-SSP) was demonstrated for MSI. ${ }^{172-173}$ The imaging capability of ambient laser ablation LMJ-SSP was demonstrated using ink lines, letters, and fingerprints on microscope slides as well as biological tissue sections with approximately $100 \mu \mathrm{m}$ lateral resolution using a $337 \mathrm{~nm} \mathrm{~N} \mathrm{~N}_{2}$ laser in transmission mode geometry. ${ }^{124,172-173}$ Recently, a commercial Leica laser inverted microscope 
microdissection system was used in transmission geometry for laser ablation sample capture into a flowing solvent followed by quadrupole time-of-flight mass spectrometer. ${ }^{174}$ A $349 \mathrm{~nm}$ Nd:YLF laser was focused to a spot size of $2 \mu \mathrm{m}$ and rastered across the sample at a step size as small as $0.5 \mu \mathrm{m}$. Ambient imaging of features as small as $0.6 \mu \mathrm{m}$ on a polymer sample and imaging cocaine in rat brain tissue sections at a resolution of $15 \mu \mathrm{m}$ was demonstrated. Lasers can also be used for ambient sampling by capturing the ablated material into a solvent droplet by decoupling the ionization process from sampling and then performing ionization by introducing the captured material into the ion source of a mass spectrometer. ${ }^{175-177}$ An IR laser was used with a $5 \mathrm{~ns}$ pulse duration, $2 \mathrm{~Hz}$ repetition rate, and a maximum fluence of $3 \mathrm{Jcm}^{-2}$ in reflection geometry to ablate biomolecules under ambient conditions to produce a sample plume that is captured in a solvent droplets. ${ }^{176}$ Droplet captured analytes are then either flow injected into an ESI source or deposited on a MALDI target for offline analysis. Moreover, the ability of mid-IR lasers to transfer biomolecules such as angiotensin II, bovine insulin, cytochrome c proteins and complex biological samples into solvent droplets without fragmentation was also demonstrated. ${ }^{177}$ Infrared laser ablation sample transfer (LAST) has also been used in conjunction with imaging for the analysis of biomolecules in rat brain tissue. ${ }^{178}$ The spatial distribution of phospholipids from mouse brain tissue was demonstrated using LAST with having a lateral resolution of approximately $400 \mu \mathrm{m}$.

Mass spectrometry imaging is an emerging area of chemical and biochemical analysis. However, the main technological barrier that limits MSI of biological specimens is the sampling ability and ionizing intact large biological molecules at the sub cellular level while maintaining the spatial resolution. The methods discussed so far indicate the possibility of performing MSI of large biological molecules such as proteins and peptides. It is also possible to perform 
proteomics at sub-micrometer level with mass spectrometry. However, performing both simultaneously is problematic. Therefore, bringing bimolecular imaging mass spectrometry to the sub cellular level is challenging.

\subsection{Near-Field Sampling and Imaging Mass Spectrometry}

Laser-based high lateral resolution MS techniques are indispensable analytical tools for biochemical MS analysis. Each approach described above has advantages and disadvantages depending on the particular application. One common disadvantage inherent to laser MS imaging is that the lateral resolution is limited primarily by the laser focus, which is restricted by the diffraction limit. Near-field laser ablation is an alternative for improving the lateral resolution below the classical optical limit of diffraction. ${ }^{179-180}$ In contrast to far-field optics, the resolution in near-field laser ablation is not dependent on the wavelength but rather from the geometry and dimensions of the of the near-field element. Below, the basic principles of near-field optics and different approaches to near-field laser ablation couple to MS for high lateral resolution chemical imaging will be discussed in detail.

\subsubsection{Near-Field Microscopy}

The earliest discussion of a method to overcome the diffraction limit, near-field optical microscopy, was formulated in $1928 .^{181}$ Synge et al. suggested that high lateral resolution can be achieved by the illumination of samples through a sub-wavelength aperture at the apex of an opaquely coated cone. Therefore, in near-field optics, the lateral resolution $\Delta x$, no longer depends on $\lambda$ but on the dimensions of the aperture. If the distance between the aperture and surface is within few nanometers (the near-field regime), the resolution of such an optical device is not limited by optical diffraction defined by the Equation 1.1 . 
Current optical near-field methods are based on atomic force microscopy (AFM) which allows near-field element to be placed near a surface. ${ }^{182-183}$ AFM has become one of the most commonly used surface probing methods in scanning probe microscopy mainly due to its versatility, reliability and ease of use. ${ }^{184-188}$ The AFM was invented by Binnig et al. in 1986, as a solution for investigating both conductors and non-conductors at the atomic level without damaging the surface of the sample. ${ }^{189}$ Unlike scanning tunneling microscope (STM), which uses tunneling current to monitor the distance between tip and a conductive surface, AFM images are obtained by measurement of the force on a sharp tip, which is attached to a silicon based cantilever, created by the proximity to the sample surface. This measured force is then kept constant level with a feedback system. ${ }^{189-190}$

The working principle of AFM is based on the reflection of a laser beam from the flat surface of a cantilever. The reflected light is detected by a four quadrant detector. ${ }^{191}$ Deflection of the tip of the cantilever due to interaction with the surface causes it to bend or twist, resulting a position change of the reflected beam on a photodiode detector. ${ }^{192}$ By recording the differences of the intensity of the reflected beam on each segment, the system can trace the movement of the cantilever. ${ }^{193}$

A schematic of the AFM working principle is shown in Figure 1-1. The precision and sensitivity of AFM is achieved by laser deflection, in combination with a piezoelectric (PZT) control of the sample to tip distance and control feedback system. Precise movement of the PZT stage is achieved by applying a voltage. ${ }^{194}$ In general, PZT material will expand $0.1 \mathrm{~nm}$ per applied $1 \mathrm{~V}$ potential. In most AFM systems the probe is stationary and the PZT stage moves in $\mathrm{x}, \mathrm{y}$, and $\mathrm{z}$ directions. 


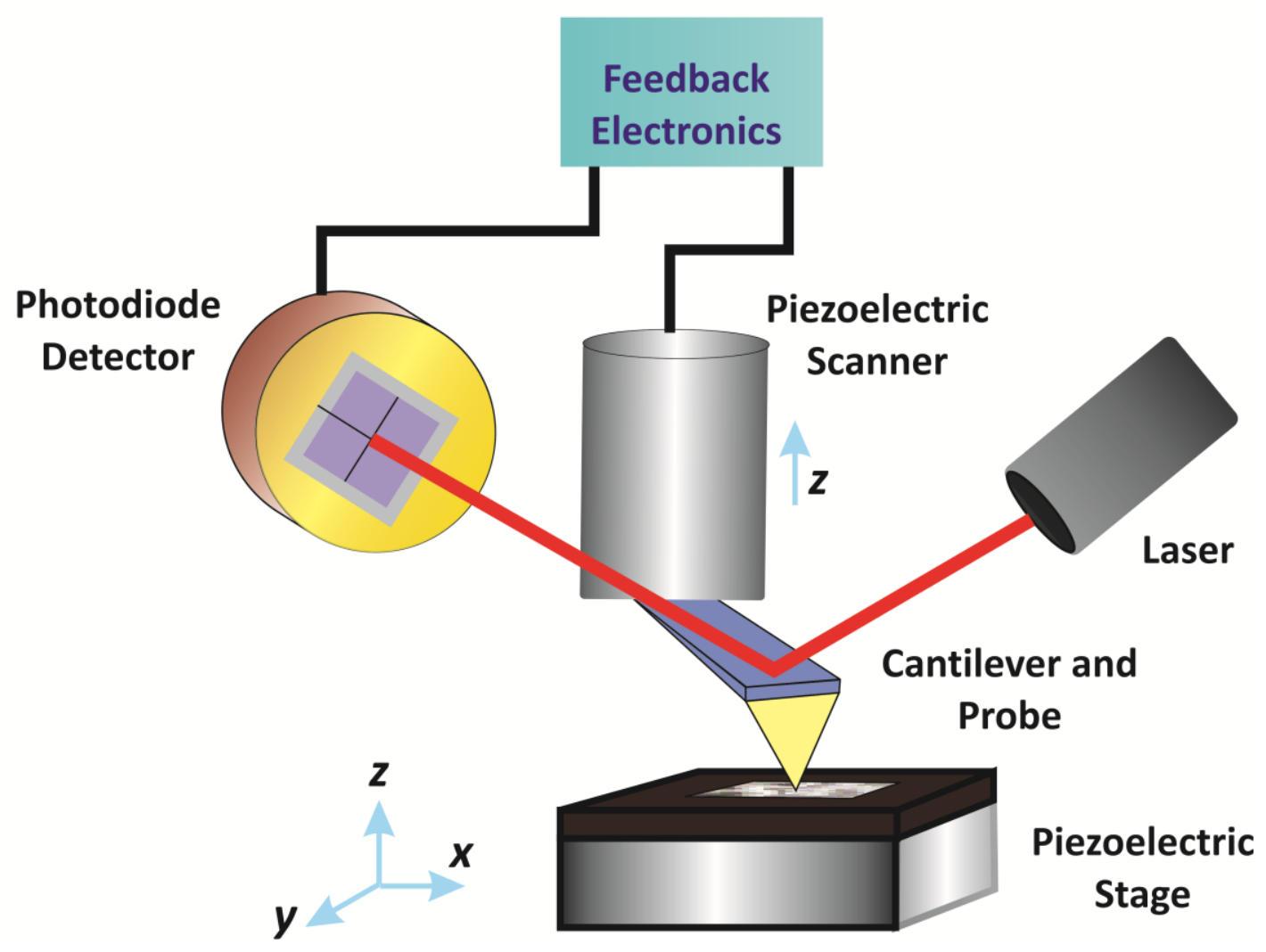

Figure 1-1 Operating principle of AFM

The sensitivity of an AFM also depends on the feedback control system. This system is used to keep the cantilever at a fixed distance from the sample surface by maintaining a set deflection of the probe. As depicted in Figure 1-1, the photodetector acquires its signal from the laser beam reflected from the cantilever. The photodiode detector signals are sent to a feedback loop that controls the cantilever deflection. When the tip is rastered parallel to the sample surface, a topographic image is acquired by fixing the tip to sample distance and recording the feedback current necessary to maintain a constant distance. ${ }^{194-195}$ 
There are several operating modes associated with AFM: tapping mode, contact mode, and non-contact mode (NC-AFM). ${ }^{196-197}$ In tapping mode AFM, the tip vibrates with a fixed amplitude near its resonant frequency. A set amplitude is defined and the surface structure is determined by the height required to keep the tip oscillation at the desired amplitude. ${ }^{192}$ When the tip encounters a different height, the cantilever oscillation amplitude changes and the feedback system moves the PZT stage in $\mathrm{z}$ direction to maintain the set amplitude. A schematic representation of the tapping mode AFM is shown in Figure 1-2.
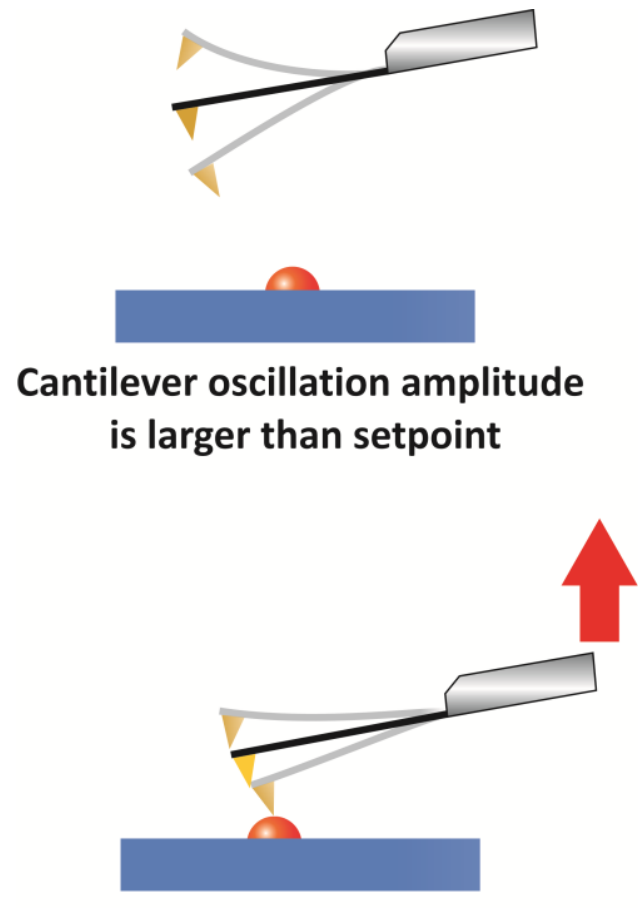

Feedback lifts the $\mathrm{Z}$ piezo. Amplitude returns to setpoint

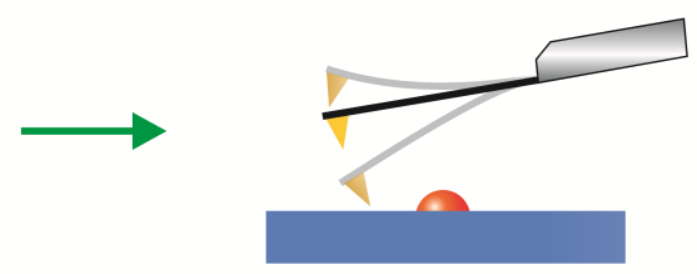

Tapping on surface. Cantilever oscillates at setpoint amplitude

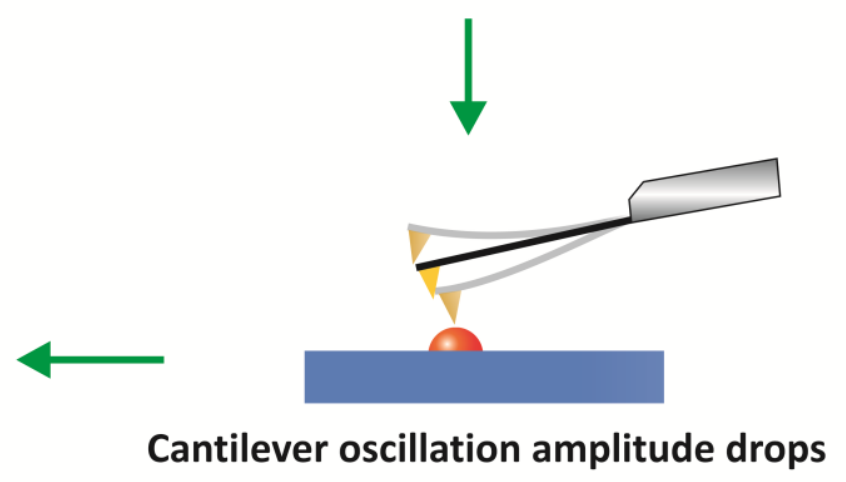

Figure 1-2. AFM cantilever motion in tapping mode 
In contact mode, the tip is brought onto the sample surface and the tip is then dragged across the sample surface with the height adjusted to maintain a constant deflection of the tip. ${ }^{198-}$ ${ }^{199}$ In NC-AFM, the tip is brought into close contact with the sample but it does not contact the surface (nm or less above the sample). A periodic oscillation of the tip near its resonant frequency is used to gauge the distance from the surface. Interaction forces are measured from cantilever frequency changes as the tip oscillation amplitude changes with the interaction force. ${ }^{197}$

When AFMs are used for near-field spectroscopy and microscopy, the procedure is called scanning near-field microscopy (SNOM). The first implementation of SNOM was in $1972 .{ }^{200} \mathrm{~A}$ line scan of a metal grating with sub-wavelength resolution was acquired by coupling light into a pulled optical fiber, which forms a sharp tip that is scanned in close distance over a sample surface. A schematic representation of near-field microscopy and coupling light through a small aperture at the tip end (aperture mode SNOM), is shown in Figure 1-3.

(a)

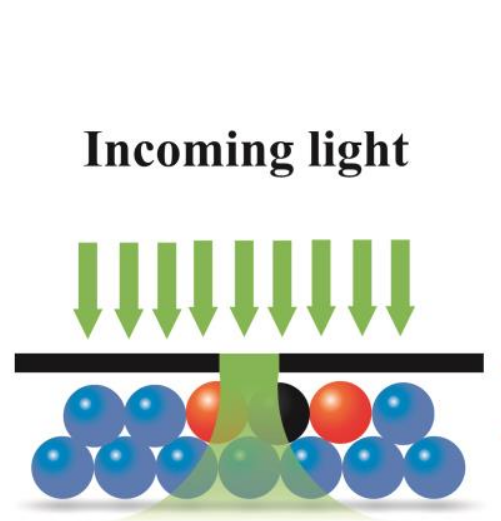

(b)

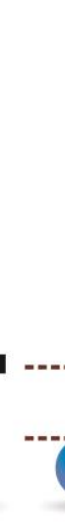

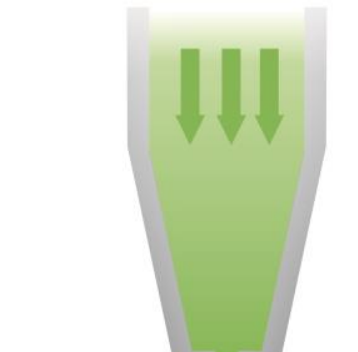

(c)

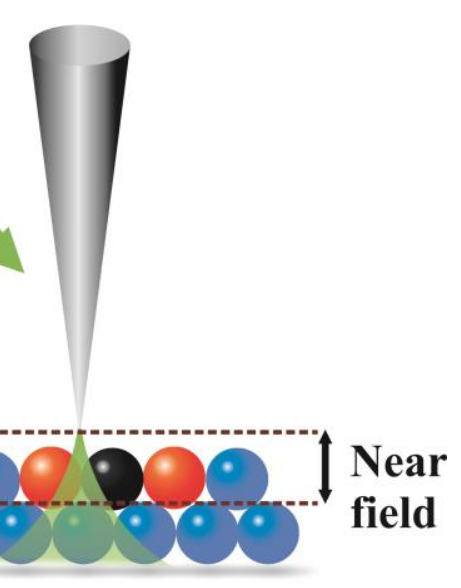

Figure 1-3. Aperture and apertureless near-field modes. Sub-wavelength resolution imaging with (a) an illuminated opaque metal screen with a small aperture in close distance over a sample (b) compared to an aperture SNOM probe having a small aperture at the tip end (c) and apertureless near-field illumination mode, where an illuminated sharp metal needle is kept in close distance over a sample. 
SNOM was mainly utilized in fluorescence and luminescence applications with lateral resolutions of few tens of nanometers. ${ }^{66}$ Near-field Raman spectroscopy using SNOM was developed by Tsai et al. ${ }^{201}$ The development of near-field Raman spectroscopy enabled SNOM Raman imaging and further improvements opened multiple avenues to utilize SNOM in chemistry and biological fields on combinations with surface enhanced Raman scattering (SERS). ${ }^{202-203}$ However, the limitations inherent to optical fiber probes hindered the use of aperture based near-field SNOM imaging technique in many fields. The concept of apertureless near-field imaging was introduced by Wessel as a solution to overcome the limitations of aperture mode near-field methods. ${ }^{204}$ A sharp metal probe or a metal-coated AFM tip is brought into close contact with a sample. When the probe interacts with the light, surface plasmons excited at the tip apex create an enhanced electric field (see Figure $1.3 \mathrm{c}$ ) ${ }^{205}$ Furthermore, the pointed probe in apertureless near-field is similar to electromagnetic antennas that enhance the propagating radiation in a confined zone. Because of this similarity, pointed metal probes used in near-field optics are also called optical antennas. ${ }^{205-206}$

\subsubsection{Near-Field Ablation and Mass Spectrometry}

Near-field optics can be used not only for spectroscopic applications ${ }^{207-209}$ but also with laser desorption and ablation. Laser energy can be delivered through a SNOM probe in aperture mode or a sharp metal probe can be irradiated by a laser in apertureless mode to ablate material from the sample surface. Near-field laser ablation coupled to mass spectrometry provides a suitable platform to analyze materials at higher lateral resolution. The feasibility of a MS analysis after ablation through an NSOM probe was first demonstrated in $1998 .^{210}$ A SNOM head was placed in the ion source of a TOF-MS and $337 \mathrm{~nm}$ wavelength laser pulses were used to irradiate the sample in aperture mode. The distribution of acetyl choline/dihydroxybenzoic 
acid deposited on a TEM grid was determined with line scans on the sample surface. The lateral resolution with this approach was approximately $1 \mu \mathrm{m}$, due to the limitations of operating the SNOM in vacuum.

Near-field laser ablation of anthracene using an SNOM tip was demonstrated in $1996,{ }^{211}$ and coupling MS with ambient near-field laser ablation was demonstrated in $2001 .^{212}$ A 1.5 attomole quantity of anthracene was sampled from a $200 \mathrm{~nm}$ diameter by $20 \mathrm{~nm}$ deep ablation crater using a $355 \mathrm{~nm} \mathrm{Nd:YAG} \mathrm{laser} \mathrm{coupled} \mathrm{to} \mathrm{a} \mathrm{NSOM} \mathrm{fiber} \mathrm{with} \mathrm{a} 170 \mathrm{~nm}$ diameter aperture. The ablated material was directed into an electron ionization (EI) source of a quadruple MS for detection and topographic images of the ablation crates were also obtained using NSOM tip. ${ }^{212}$ Although, sub-micrometer sampling and MS detection were demonstrated, EI used in this method limits the molecules that can be detected with this system. In 2008, an ion-trap/TOF MS was used to achieve higher detection sensitivity. ${ }^{213}$ A schematic representation of the experimental configuration is shown in Figure 1-4. The laser ablation was ambient and the ablated material was transported through a heated capillary into the mass spectrometer. ${ }^{213}$ Mass spectra from a $2 \mu \mathrm{m}$ wide and $800 \mathrm{~nm}$ deep ablation craters were acquired for both anthracene and 2,5-dihydrobenoic acid (DHB). The feasibility of acquiring localized chemical information along with topographical imaging of the same sample by scanning the tip over the sample was demonstrated using near-field laser ablation and mass spectrometry.

Post-ionization of the near-field ablated material allowed ionization to be performed independently from material desorption. ${ }^{213}$ Near-field laser desorption and electron ionization post-ionization was accomplished using a SNOM apparatus and quadrupole mass spectrometer. A $355 \mathrm{~nm} \mathrm{Nd:YAG} \mathrm{laser} \mathrm{was} \mathrm{coupled} \mathrm{into} \mathrm{a} \mathrm{near-field} \mathrm{optical} \mathrm{fiber} \mathrm{SNOM} \mathrm{tip} \mathrm{and} \mathrm{the} \mathrm{laser}$ 


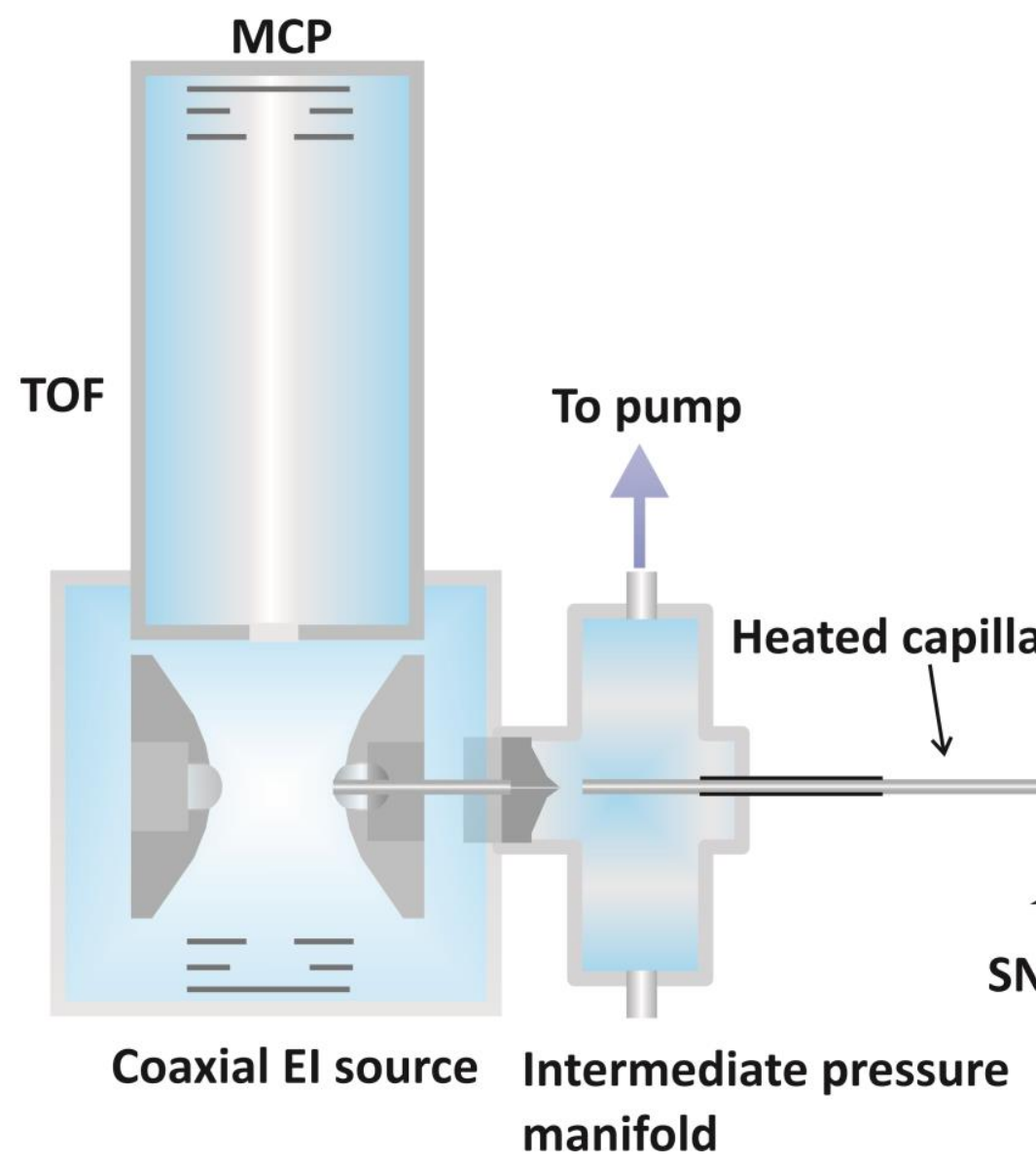

UV laser

SNOM tip

SNOM xyz sample stage

\section{Coaxial El source Intermediate pressure manifold}

Figure 1-4. A schematic of aperture mode near-field laser ablation- MS system. A SNOM tip is coupled with a pulsed laser and place on optical near-field to ablate material.

ablated material was sampled into a $20 \mathrm{~cm}$ long and $20 \mu \mathrm{m}$ ID stainless steel capillary that was positioned $5 \mu \mathrm{m}$ from the tip. Nitrogen gas was detected from the laser-induced decomposition of bis(phenyl-N,N-diethyltriazene) from $200 \mathrm{~nm}$ near-field ablation spots. A similar apparatus used a $2 \mathrm{kHz}$ repetition rate $349 \mathrm{~nm} \mathrm{Nd:YLF}$ coupled into a SNOM tip with laser desorbed neutrals sampled into an ion trap mass spectrometer with a $250 \mu \mathrm{m}$ ID capillary. ${ }^{213}$ Tips with 500 to $800 \mathrm{~nm}$ diameter apertures produced craters as small as $200 \mathrm{~nm}$ diameter. Spatially resolved images of anthracene at $5 \mu \mathrm{m}$ lateral resolution were reported. It was found that much of the 
ejected material was redeposited as particulate ${ }^{214}$ and the overall efficiency of ion production was $10^{-4} \cdot 215$

An apertureless near-field LA-ICP-MS method for acquiring localized elemental distribution with nanometer scale resolution was demonstrated by Becker and coworkers. ${ }^{216-219}$ The possibility of ablating material using a sharp silver needle with $\sim 100 \mathrm{~nm}$ diameter in apertureless near-field mode was demonstrated. A schematic of the experimental configuration is shown in Figure 1-5. An etched silver wire with a radius between $100 \mathrm{~nm}$ and $250 \mathrm{~nm}$ was

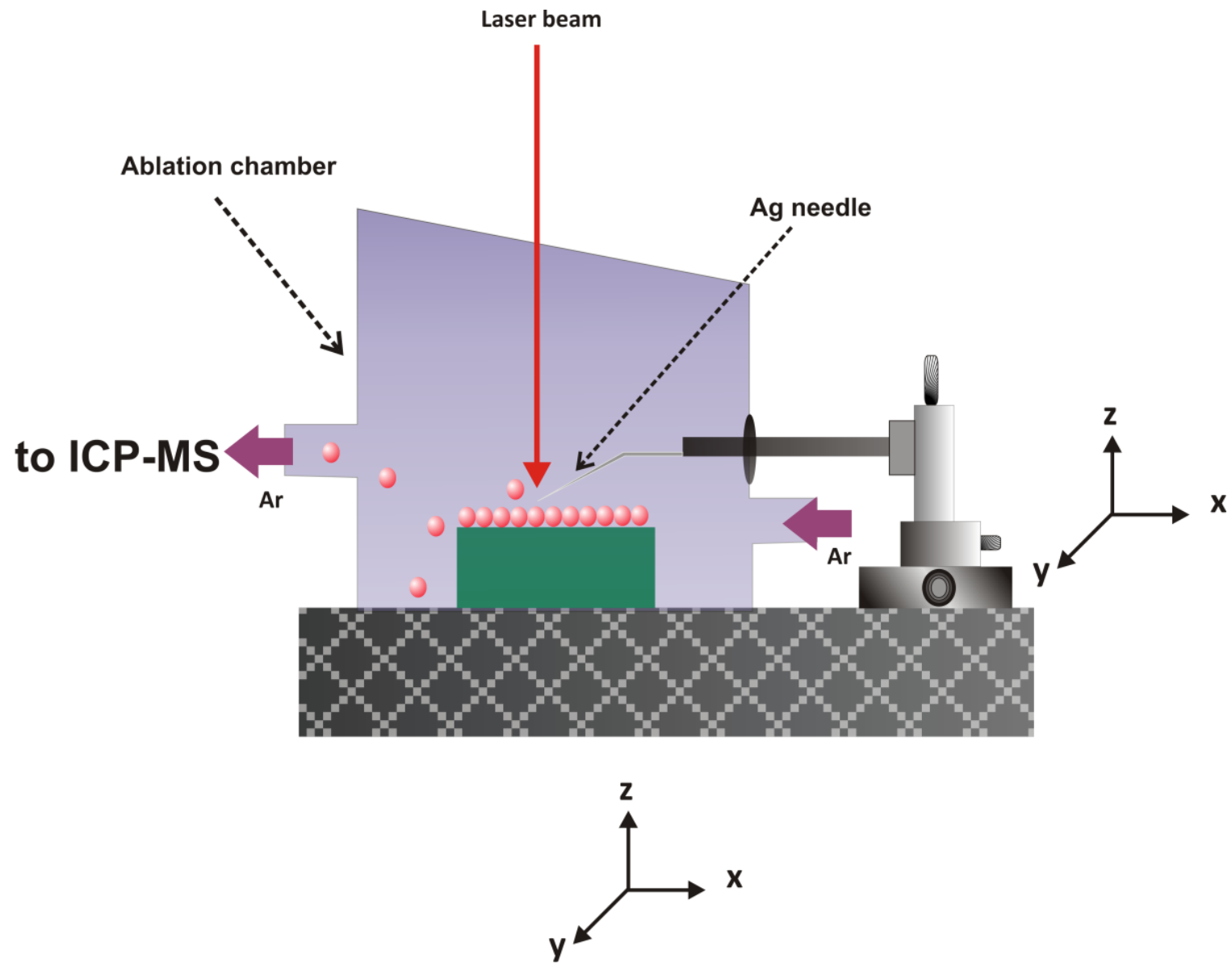

Figure 1-5. Apertureless near-field laser ablation ICP-MS system 
positioned about $200 \mathrm{~nm}$ above the sample surface using a precise $X Y Z$ manipulator, and frequency doubled $532 \mathrm{~nm} \mathrm{Nd:YAG} \mathrm{laser} \mathrm{was} \mathrm{focused} \mathrm{on} \mathrm{to} \mathrm{the} \mathrm{silver} \mathrm{wire.} \mathrm{In} \mathrm{their} \mathrm{initial}$ experiments, tunnel current was used to control the tip sample distance. Hence, this analysis was limited to samples with good electrical conductivity. ${ }^{216,220}$ Ablation was carried out in a custom made ablation chamber and the ablated material was transported with argon as carrier gas into the ICP-MS. The figures of merit of the NF-LA-ICP-MS method, tip diameter, and tip sample distance were also explored with different sample surfaces. ${ }^{221}$ Although higher resolution was achieved, it is not clear that it is a result of near-field enhancement due to the relatively large distance between the tip and surface. ${ }^{222}$

Goeringer et al. demonstrated ambient laser desorption ionization using a metal AFM probe to in apertureless near-field mode. ${ }^{223}$ A schematic representation of the apparatus is shown in Figure 1-6.

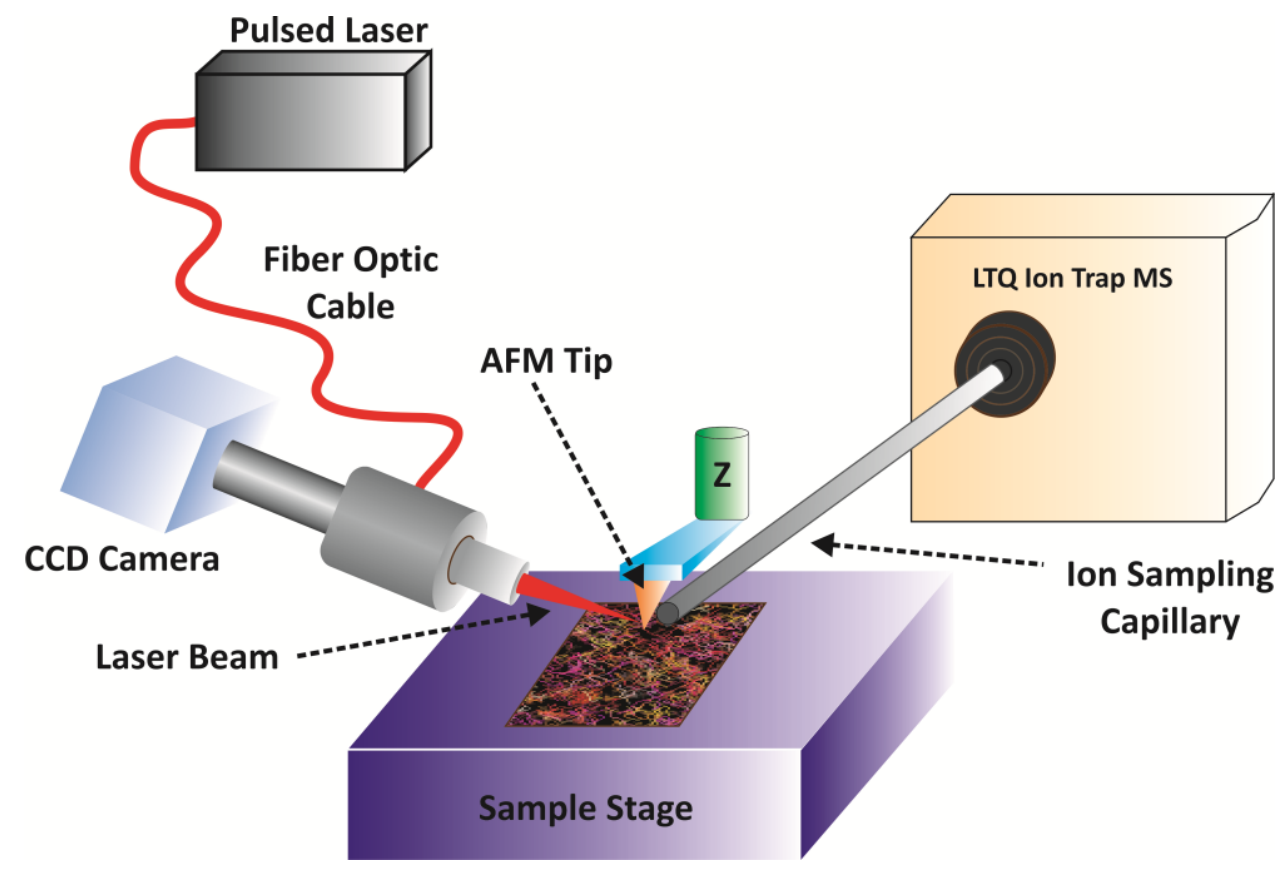

Figure 1-6. Schematic of combined AFM and LDI-MS imaging system 
In these experiments, a frequency doubled $532 \mathrm{~nm} \mathrm{Nd:YAG} \mathrm{laser} \mathrm{was} \mathrm{focused} \mathrm{at} \mathrm{the} \mathrm{tip}$ of a gold coated AFM probe, and the tip was positioned within $10 \mathrm{~nm}$ from the sample to ablate material. Ablation craters $50 \mathrm{~nm}$ in diameter were observed on rhodamine B dye surface. ${ }^{223} \mathrm{In}$ this study, the near-field enhancement factor was determined using the ratio of far field damage threshold to the near-field ablation threshold, found to be in the order of $10^{2}$.

Recently, the utility of near-field laser ablation using an AFM probe for ambient nanoscale sampling and acquiring both topographical and chemical images via MS was demonstrated. ${ }^{224}$ In this approach, a capillary connected to the mass spectrometer (see Figure 16) sampled the desorbed ions into the ion trap mass spectrometer. Chemical images were obtained with $2 \mu \mathrm{m}$ lateral resolution and AFM topographic images with sub-micrometer resolution of the same surface were demonstrated.

Near-field laser ablation methods discussed in this section indicate the feasibility of ablating material at sub-micrometer scale. Hence, efficient capturing of the ablated material, post sample preparation steps to pre-concentrate prior to analysis, and mass spectrometers with suitable ionization will enable biomolecule sampling and imaging at high lateral resolution. The development of new methods combining apertureless near-field techniques and ambient sampling that makes sampling a possibility on the sub-micrometer scale to acquire localized chemical information in conjunction with MS.

\subsection{Research Objectives}

The objective of this research was to develop a near-field laser ablation method capable of sampling large biological molecules for MS. The approach is based on apertureless near-field laser ablation sampling with capture of the material for ionization and MS detection. The work presented in this dissertation is the first step toward sub-micrometer scale sampling of single 
cells and tissue for MS detection. The AFM tip-enhanced laser ablation system described in Chapter 2 was designed and sub-micrometer scale sampling, capture and detection of small molecules and biomolecules were demonstrated using the AFM tip-enhanced laser ablation system. The experiments discussed in Chapter 3 were performed with a $355 \mathrm{~nm}$ nanosecond pulsed Nd:YAG laser, which was able to ablate peptides and proteins with minimal fragmentation from micrometer or smaller spots. The expansion of the laser wavelengths tested from the UV to the visible and infrared wavelengths are described in Chapters 4 and 5 . The figures of merit of the AFM tip-enhanced laser ablation system, laser wavelength, polarization, fluence, limit of detection, and near-field laser ablation efficiency were also explored. In Chapter 4, a proof of concept test for tissue near-field laser ablation for MS is described. 


\section{CHAPTER 2. INSTRUMENTATION}

This chapter contains a brief discussion of the instruments and procedures used in this research. A detailed explanation of coupling AFM with laser ablation for near-field laser ablation is presented. System parameters for sub-micrometer scale sampling and mass analysis are provided.

\subsection{Atomic Force Microscope}

The work presented in this dissertation used an AFM system for placing a metal coated probe to laser ablate material in apertureless mode and to acquire topographical images of the ablated area of the sample. To perform tip-enhanced laser ablation, precise positioning of the probe is critical. The tip must be irradiated with an external laser. Therefore, a custom AFM was used for all of these experiments. The working principle of a typical AFM instrument and its different operational modes are discussed in detail in Chapter 1.

\section{Anasys afm+}

Figure 2-1 is a photograph of the AFM-tip-enhanced laser ablation system. All topographical images of the sample of interest and precise positioning of the tip were achieved by using an atomic force microscope (Anasys, Santa Barbara, CA). The system is based on a commercial system (afm+) that was modified to allow open access to the AFM stage but is otherwise identical. The afm+ system includes a brightfield optical microscope with spatial resolution of $1.5 \mu \mathrm{m}$ to see the location of the probe on the sample surface and a motorized closed loop XYZ piezoelectric stage to position the probe on the sample with $<1 \mu$ m spatial resolution. The system allows probe to be manually positioned at a number of locations or alternatively the system also capable of measuring the sample over an area of $100 \times 100 \mu \mathrm{m}$ on the surface using the array function. The system allows obtaining topographical images in all 
common imaging modes with high spatial resolution imaging typically in the $<10 \mathrm{~nm}$ range spatial resolution depending on the tip radius of the probe being used. The Anasys afm+ system is also capable of performing nanoscale localized thermal analysis. ${ }^{225}$ These measurements are made by bringing a probe capable of being heated into contact with sample. The thermal probes can be resistively heated by flowing current through a conductive path in the cantilever.

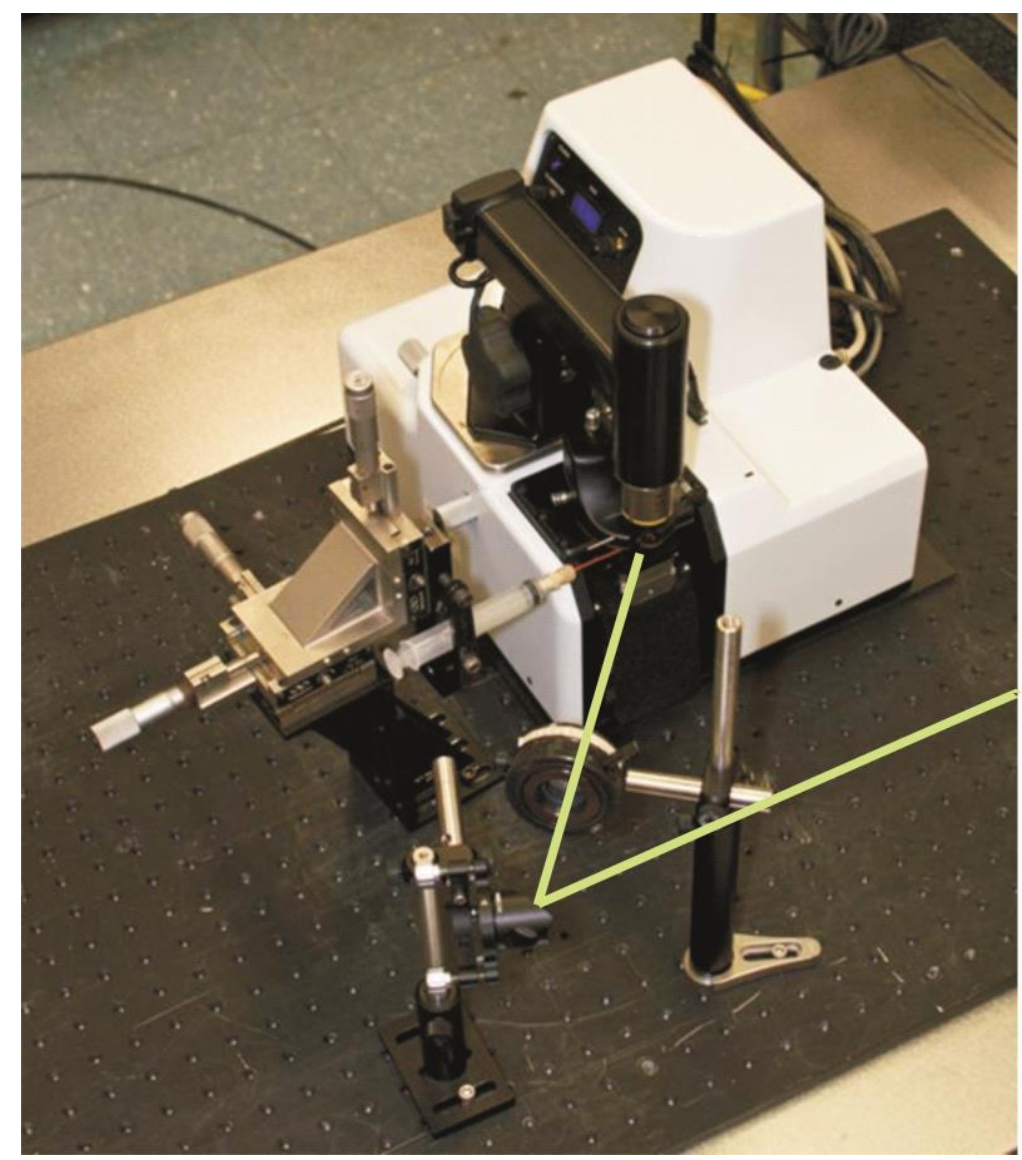

Figure 2-1. Photograph of the AFM tip-enhanced laser ablation system. The laser beam path (indicated by a green line) reflected by the mirror, travels through the focusing lens and irradiates the AFM probe. 


\section{Gold-coated AFM Tips}

For all experiments, gold-coated silicon probes (ACCESS-NC-GG, Applied Nanostructures, Mountain View, CA) with an apex half-cone angle of $11^{\circ}$ and a radius of curvature of $30 \mathrm{~nm}$ was used. The nominal values of spring constant and resonant frequency were $78 \mathrm{~N} / \mathrm{m}$ and $300 \mathrm{kHz}$, respectively. These probes are designed with a tilt angle of $17^{\circ}$ with respect to the cantilever axis, which allows direct optical view of the tip when imaging and irradiating with an external laser. The reflective sides of the probe are coated with gold with 15 nm thickness, helps to enable optical antenna effect when irradiated by an external user. A closeup photograph of the AFM sampling stage and tip is shown in Figure 2-2.

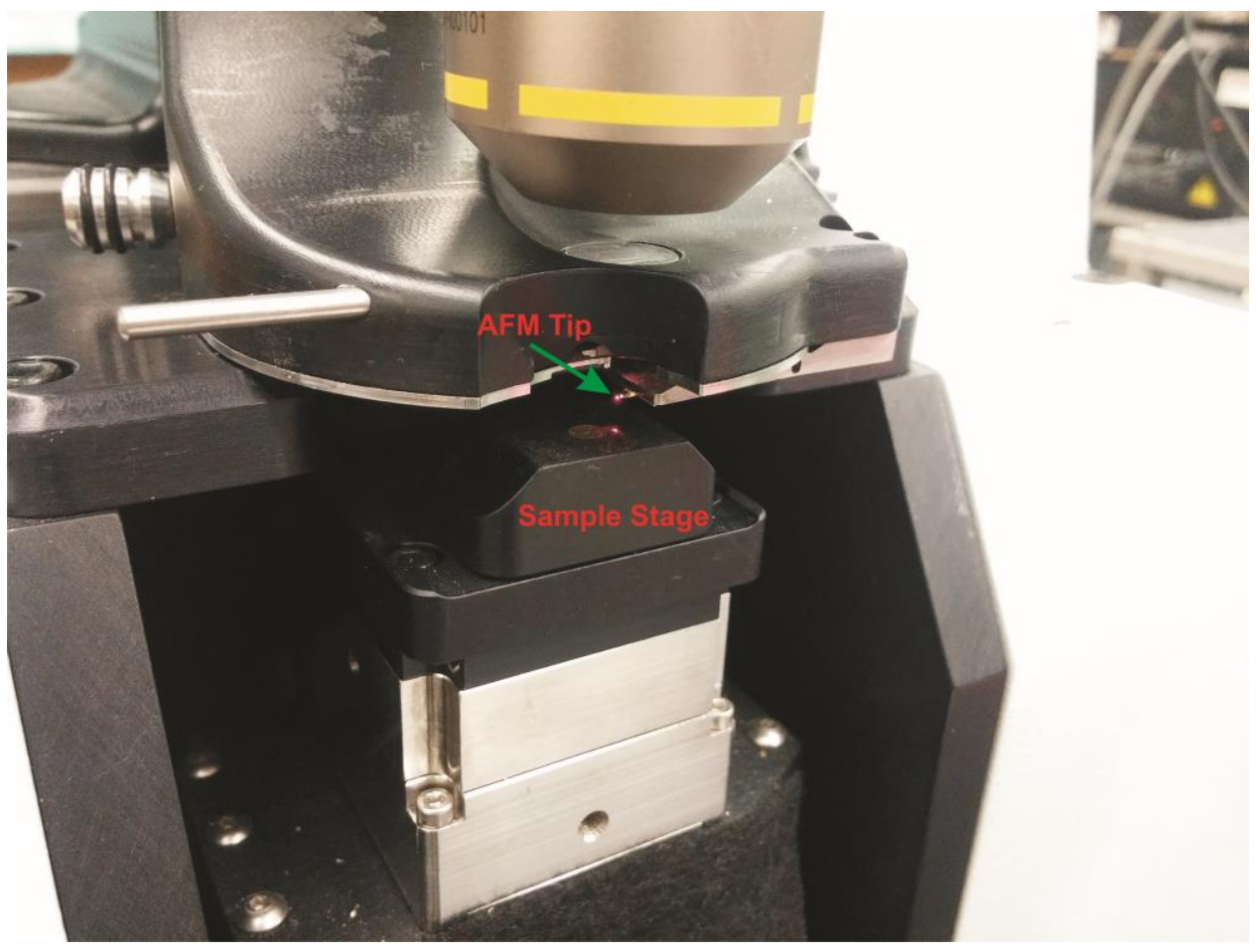

Figure 2-2. Photograph of the sampling stage and tip of afm+ instrument 
Prolong exposure of the AFM probe to the laser tends to cause damage by removing the gold coating. Figure 2-3 is a SEM image of the AFM tip before and after exposure to the external laser for 1000 laser shots.
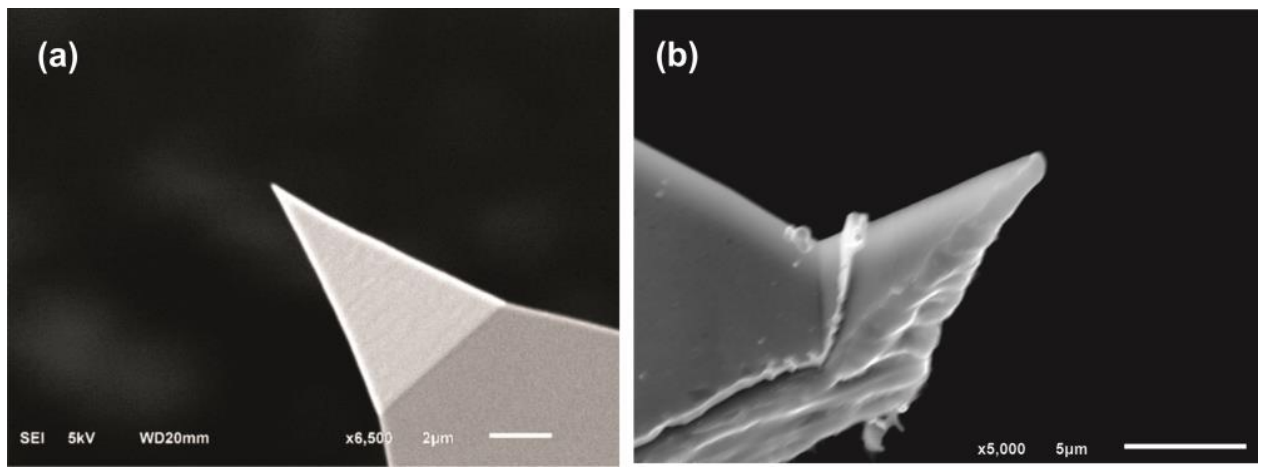

Figure 2-3. SEM images of the AFM probe (a) before use and (b) damaged after exposure to 1000 laser shots.

\subsection{Laser and Optical System}

The pulsed laser source used for the ablation is a Q-switched, Nd:YAG system (Powerlite 8000, Continuum, San Jose, CA), that can be operated with a second and a third harmonic generator. Therefore, desired output wavelength except the fundamental (1064 nm) was chosen (532 $\mathrm{nm}$ and $355 \mathrm{~nm}$ ) by changing the frequency doubling and tripling crystals respectively. The pulse duration was $5 \mathrm{~ns}$. The pulse energy of the laser was controlled by shifting flash lamp voltage, which will change the time between the ignition of the flash lamp. Further adjustment to the laser energy was done using a variable neutral density filter. As depicted in Figure 2-4, desired polarization of the output beam was adjusted using a half wave plate and the laser beam was mildly focused using a $25 \mathrm{~cm} \mathrm{CaF}_{2}$ lens to irradiate the AFM probe at an angle of $83^{\circ}$, nearly 
parallel with the laser table. The laser was directed toward the AFM at an angle of $45^{\circ}$ with respect to the vertical plane containing the cantilever.

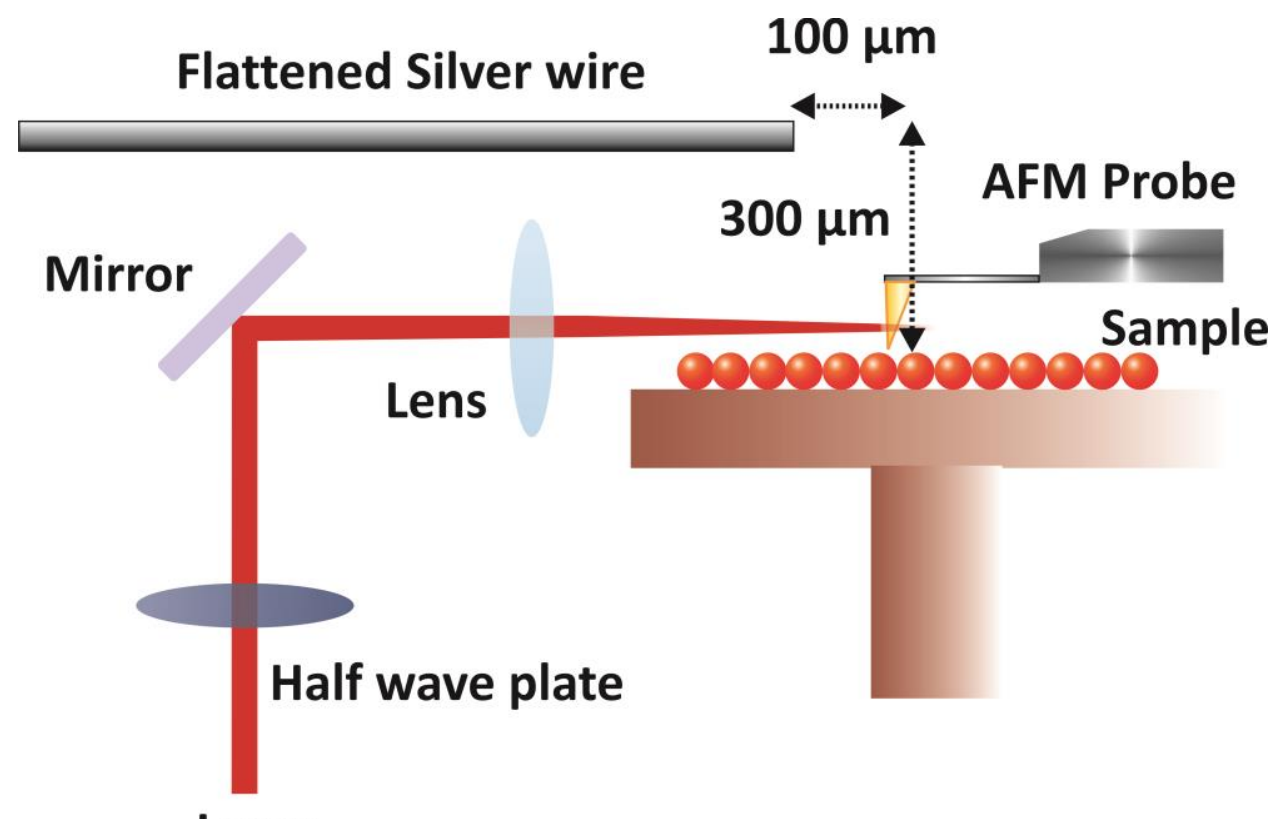

\section{Laser}

Figure 2-4. Schematic of the AFM tip-enhanced laser ablation system

\section{Material Capture}

The ablated material was captured on a silver wire with a diameter of $100 \mu \mathrm{m}$. The wire was flattened manually at the tip to produce a ribbon $400-500 \mu \mathrm{m}$ in width. The wire was mounted on a three-axis mechanical stage and positioned above the AFM tip. A close-up photograph of the carefully positioned silver wire is shown in Figure 2-5. The distance was viewed using the AFM video camera and the distance between the capture wire and the ablation spot was set to approximately $100 \mu \mathrm{m}$. The height of the wire above the sample surface was approximately $300 \mu \mathrm{m}$. For multiple spot collection, the wire was kept in the same position above the tip. The tip-sample distance was controlled by engaging the AFM probe in tapping 
mode at an amplitude set point of $1 \mathrm{~V}$, where the amplitude sensitivity of the probe was 6-7 $\mathrm{nm} / \mathrm{V}$. Hence, the AFM probe was oscillating between $0-15 \mathrm{~nm}$ from the surface at its resonance frequency during the laser irradiation.

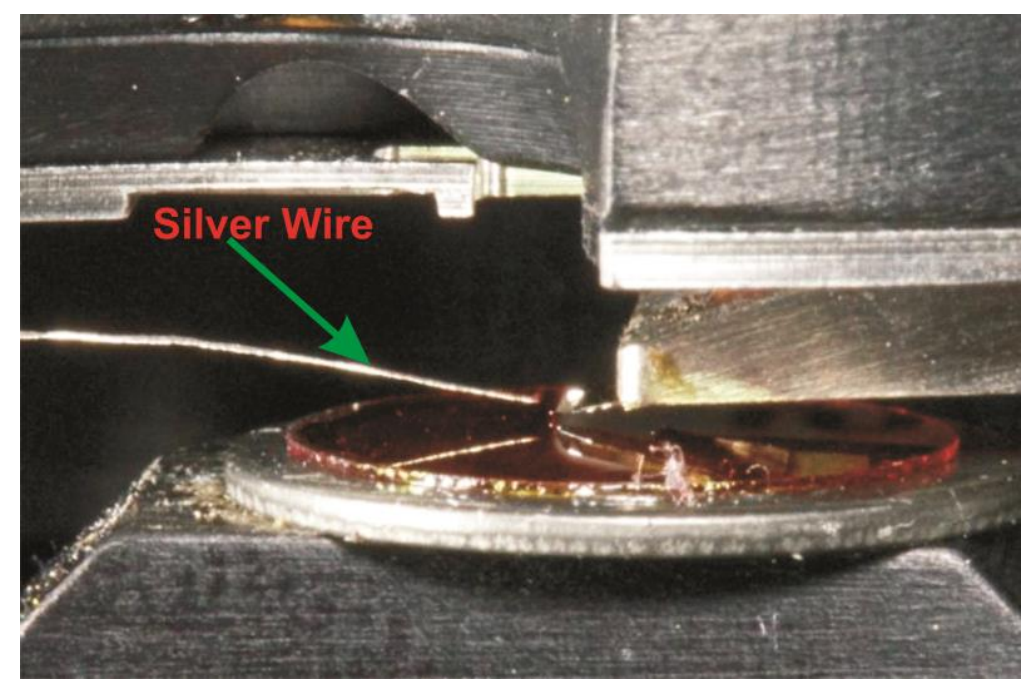

Figure 2-5. Mounted silver wire positioned $300 \mu \mathrm{m}$ above the AFM tip

\section{Mass Spectrometer}

For the experiments reported in this dissertation, all the mass spectra were taken using a MALDI TOF mass spectrometer (Ultraflextreme; Bruker). This system is equipped with a frequency tripled Nd:YAG $355 \mathrm{~nm}$ laser to desorb samples. ${ }^{226}$ The laser can be operated at $1 \mathrm{kHz}$ repetition rate and the spot size of the laser, typically between $10-100 \mu \mathrm{m}$, can be controlled by a computer. The MALDI TOF instrument is capable of operating in both linear and reflectron modes and the resolution of the MALDI TOF system is 40,000 in 700 to $5000 \mathrm{~m} / \mathrm{z}$ range.

The instrument was used in reflectron mode and mass spectra result from laser desorption ionization for anthracene and rhodamine $6 \mathrm{G}$ and MALDI for angiotensin II and insulin. After capture, the wire was removed from the positioning stage, cut to a $4 \mathrm{~mm}$ length and attached to a 
MALDI target using double-sided conductive tape. The laser spot diameter in the MALDI mass spectrometer was $100 \mu \mathrm{m}$ and 500 shots were used to obtain the spectrum on each spot

For MALDI mass spectrometry of angiotensin II and insulin, a saturated solution of $\alpha$ cyano-4-hydroxycinnamic acid (CHCA, Sigma-Aldrich, St. Louis, Missouri) dissolved in a mixture of 85:15 ratio of acetonitrile (99.8\%, EMD Chemicals, Gibbstown, New Jersey) and $0.1 \%$ trifluoroacetic acid (TFA, 99.0\%, Fischer Scientific, Fairlawn, New Jersey) in water was used as the matrix. After capture, the silver wire was washed in $5 \mu \mathrm{l}$ of the matrix solution and the matrix and the solution was deposited on the MALDI target. The spectrum for angiotensin II was obtained in reflectron mode whereas linear mode was used for insulin. A $100 \mu \mathrm{m}$ laser spot diameter and 500 shots were used to obtain the mass spectra.

\subsection{Tissue Samples}

A microtome was used to cut $10 \mu \mathrm{m}$ thick mouse brain tissue sections that were thawmounted on $25 \mathrm{~mm} \times 50 \mathrm{~mm}$ glass microscope slides and were stored at $-80{ }^{\circ} \mathrm{C}$ until analysis. The samples were dried in a vacuum chamber for 2 hours prior to the analysis. The glass microscope slide with the tissue section was placed on AFM sample stage prior to the experiments. The AFM images of the area of interest were obtained prior to the TELA and after the ablation experiments for comparison. All AFM topographic images shown in this work were obtained in tapping mode.

The ablated material was extracted from the capture wire by immersing the tip of the wire in $2 \mu \mathrm{L}$ of a saturated solution of $\alpha$-cyano-4-hydroxycinnamic acid (CHCA, Sigma-Aldrich, St. Louis, Missouri) matrix in a 50:50 (v/v) solution of acetonitrile and 0.1\% aqueous trifluoroacetic acid. The solution was then deposited on a MALDI target (MTP 384, Bruker) for analysis. Mass spectra were acquired from an average of 500 laser shots. 
Lipids were extracted from the rat brain tissue using the Folch method ${ }^{227-228}$ for comparison with the ablation capture samples. Rat brain tissue sections were homogenized in 1 $\mathrm{mL}$ of 2:1 dichloromethane:methanol and the mixture was vortexed for $15 \mathrm{~min}$. Solids were removed by a $0.45 \mu \mathrm{m}$ filter (Durapore, $0.45 \mu \mathrm{m}$, Cole-Parmer, IL, USA) and $0.2 \mathrm{~mL}$ of a 0.15 $\mathrm{M} \mathrm{NaCl}$ solution was added to the filtrate and the mixture was centrifuged at $2000 \mathrm{rpm}$ for 10 min. The organic layer containing the lipids was decanted and evaporated under vacuum in a rotary evaporator for $10 \mathrm{~min}$. The lipids were resuspended in $2 \mu \mathrm{L}$ of CHCA matrix solution and deposited on a MALDI target for analysis.

HPLC grade acetonitrile and methanol were purchased from Fisher Scientific (Pittsburgh, PA, USA). Dicloromethane, TFA and $\mathrm{NaCl}$ were purchased from Sigma-Aldrich (St. Louis, MO, USA). Rat brain tissue samples were donated by the National Institute of Drug Abuse intramural research program. (National Institutes of Health, Baltimore, Maryland, USA) 


\section{CHAPTER 3. TIP-ENHANCED LASER ABLATION SAMPLE TRANSFER FOR SMALL AND LARGE MOLECULE MASS SPECTROMETRY*}

The purpose of the research described in this chapter was to explore the utility of AFM tip-enhanced laser ablation mass spectrometry for the detection of biomolecules. Atomic force microscope (AFM) tip-enhanced laser ablation was used to transfer molecules from thin films to a suspended silver wire for off-line mass spectrometry using laser desorption ionization (LDI) and matrix-assisted laser desorption ionization (MALDI). For the small molecules anthracene and rhodamine $6 \mathrm{G}$, the wire was cut and affixed to a metal target using double-sided conductive tape and analyzed by LDI using a commercial laser desorption time-of-flight mass spectrometer. For the larger polypeptide molecules angiotensin II and bovine insulin, the captured material was dissolved in saturated matrix solution and deposited on a target for MALDI analysis. Approximately $100 \mathrm{fg}$ of material was ablated from each of the $1 \mu \mathrm{m}$ ablation spots and transferred with approximately $3 \%$ efficiency.

\subsection{Introduction}

The use of tip-enhanced or near-field ablation for the detection of biomolecules requires efficient coupling to a soft ionization method such as MALDI or electrospray. In particular, the method must be efficient at forming ions from clusters and small particles that are produced by near-field ablation. One approach is to merge the ablated material with an electrospray source. 148, 229-230 This technique has been used with a unique etched optical fiber configuration for infrared laser ablation imaging. ${ }^{144}$ The spatial resolution observed was $20 \mu \mathrm{m}$ and, although an

\footnotetext{
* Some portions of the work reported in this chapter was previously appeared as Ghorai, S.; Seneviratne, C. A.; Murray, K. K., Tip-Enhanced Laser Ablation Sample Transfer for Biomolecule Mass Spectrometry, and has been published in the Journal of the American Society for Mass Spectrometry. ${ }^{231}$ Reprinted by permission of the Elsevier.
} 
optical fiber was used, ablation conditions were not in the near-field regime. An alternative approach that does not require merging of the ablation plume with the electrospray is laser ablation sample transfer. ${ }^{172,176,232}$ In this approach, the ablated material is captured in static or flowing solvent or on a solid surface, after which, MALDI or electrospray can be performed. Decoupling the sampling and ionization steps allows both to be optimized independently and has the potential for high efficiency sample utilization.

In this chapter, tip-enhanced laser assisted sample transfer using an atomic force microscope to position a conductive probe for UV laser ablation is described. A $355 \mathrm{~nm}$ pulsed laser was focused onto a gold-coated silicon AFM tip. The desorbed or ablated material was captured on a silver wire suspended above the AFM tip and the wire was removed for analysis directly by LDI or with the addition of a matrix for MALDI using a commercial time-of-flight mass spectrometer. Removal of material with sub-micrometer level lateral resolution, topographical images of the ablation craters, LDI, and MALDI detection of the captured material were demonstrated. The system is able to transfer fmol quantities of molecules as large as peptides and small proteins with little to no fragmentation.

\subsection{Experimental}

The AFM laser ablation sample transfer configuration described in Chapter 2 was used in all experiments reported in this section. The ablation laser is a $355 \mathrm{~nm}$ frequency tripled pulsed Nd:YAG laser with a repetition rate of $10 \mathrm{~Hz}$ and $5 \mathrm{~ns}$ pulse width. The $355 \mathrm{~nm}$ wavelength was chosen for comparison to several of the previous near-field ablation studies. ${ }^{212-215}$ The polarization of the beam was adjusted using a half wave plate and polarization perpendicular to the tip axis was used in all of the experiments described below. Although optimum tip enhancement typically requires polarization parallel to the tip axis of a pointed probe ${ }^{67,233}$ the 
tip used in this work is a triangular pyramid that could favor enhancement at the pyramid edges, similar to what has been observed for triangular nanoprisms. ${ }^{234-237}$ The beam was mildly focused using a $25 \mathrm{~cm} \mathrm{CaF}_{2}$ lens to irradiate the $\mathrm{AFM}$ probe at an angle approximately $80^{\circ}$ from normal, nearly parallel with the laser table. The laser was directed toward the AFM tip at an angle of $45^{\circ}$ in the horizontal plane and approximately $10^{\circ}$ in the vertical plane. The focal point was approximately $4 \mathrm{~cm}$ beyond the probe and the resulting laser diameter where it intersected the tip was $600 \mu \mathrm{m}$. The efficiency of ablation was found to be highly dependent both on the laser focus and angle with respect to the AFM tip.

Tip-to-surface control for laser ablation was accomplished by engaging the tip in tapping mode with the sample at an amplitude set point of $1 \mathrm{~V}$, where the amplitude sensitivity of the tip was 6-7 nm/V. The tip was operated in tapping mode with the tip-to-sample distance set to 15 $\mathrm{nm}$, thus the tip was oscillating between 0 and $15 \mathrm{~nm}$ while being irradiated. Height control with a static tip was not possible with the current AFM control software. Ablation was performed using 30 laser shots over 3 s per ablation spot. The AFM conditions were the same for all of the material removal reported below.

The ablated material was captured on a silver wire with a diameter of $100 \mu \mathrm{m}$. The wire was flattened manually at the tip to produce a ribbon $400-500 \mu \mathrm{m}$ in width. The wire was mounted on a three-axis mechanical stage and positioned above the AFM tip. The distance was viewed using the AFM video camera and the distance between the capture wire and the ablation spot was set to approximately $100 \mu \mathrm{m}$. The height of the wire above the sample was approximately $300 \mu \mathrm{m}$. For multiple spot collections, the wire was kept in the same position above the tip. A new wire was used for each set of collections. 
For all off-line LDI experiments, the wire was removed from the positioning stage, cut to a $4 \mathrm{~mm}$ length and attached to a MALDI target using double-sided conductive tape and for MALDI experiments, the silver wire was washed in $5 \mu \mathrm{L}$ of the matrix solution and the matrix and extracted analyte was deposited on the MALDI target plate prior to off-line MALDI analysis as described in Chapter 2.

To prepare thin films for ablation, anthracene was dissolved in acetonitrile at a concentration of $1 \mathrm{mg} / \mathrm{ml}$ and a similar concentration solution of rhodamine $6 \mathrm{G}$ (99\%, SigmaAldrich) was prepared in dichloromethane. Angiotensin II and bovine insulin were prepared in equal volumes of acetonitrile and $0.1 \%$ aqueous TFA. The thin film of the sample was prepared by slow evaporation of 3 drops of solution placed on glass cover slip.

\subsection{Results}

Initial studies used tip-enhanced transfer of material to a silver wire that was attached to a target for direct laser desorption ionization mass spectrometry analysis. The AFM system was first used to irradiate an anthracene thin film prepared from an acetonitrile solution. Figure 3-1 a shows the AFM image of anthracene thin film before laser irradiation. The film roughness was found to be $3.4 \mathrm{~nm}$, measured over the $1 \mu \mathrm{m}$ area of Figure 3-1 a. Material removal was performed by directing the laser onto the probe for 3 seconds with a repetition rate of $10 \mathrm{~Hz}$ at a fluence of $850 \mathrm{~J} / \mathrm{m}^{2}$ with the tip held $15 \mathrm{~nm}$ above the surface as described above. A representative image of the crater created using 30 laser shots is shown in Figure 3-1 b. The spots were elongated in the dimension perpendicular to the laser beam with a dimension of $c a 1 \mu \mathrm{m} \times$ $0.6 \mu \mathrm{m}$. The height profile of the crater along the line indicated in Figure 3-1 b is shown in Figure 3-1 c. The average depth of the ablation craters under the conditions used for Figure 3-1 was $200 \mathrm{~nm}$ and the average ablation spot area was $0.5 \mu \mathrm{m}^{2}$. This corresponds to $40 \mathrm{fg}$ of 
material removed from the thin film, assuming a conical crater. A $200 \mathrm{~nm}$ wide and $40 \mathrm{~nm}$ tall rim was formed around the anthracene ablation crater, which is attributed to the buildup of molten ejecta. Ablation craters for rhodamine 6G are shown in Figure 3-1 d. The laser energy, focus, and number of shots were identical to that used to obtain Figure 3-1 b. The rhodamine ablation craters were round with an average size of $900 \times 650 \mathrm{~nm}$. An image of a single crater is shown in Figure 3-1 e and its depth profile is shown in Figure 3-1 f. The average depth of the craters was $150 \mathrm{~nm}$, which corresponds to $30 \mathrm{fg}$ of material removed. The ejecta rim is $20 \mathrm{~nm}$ tall
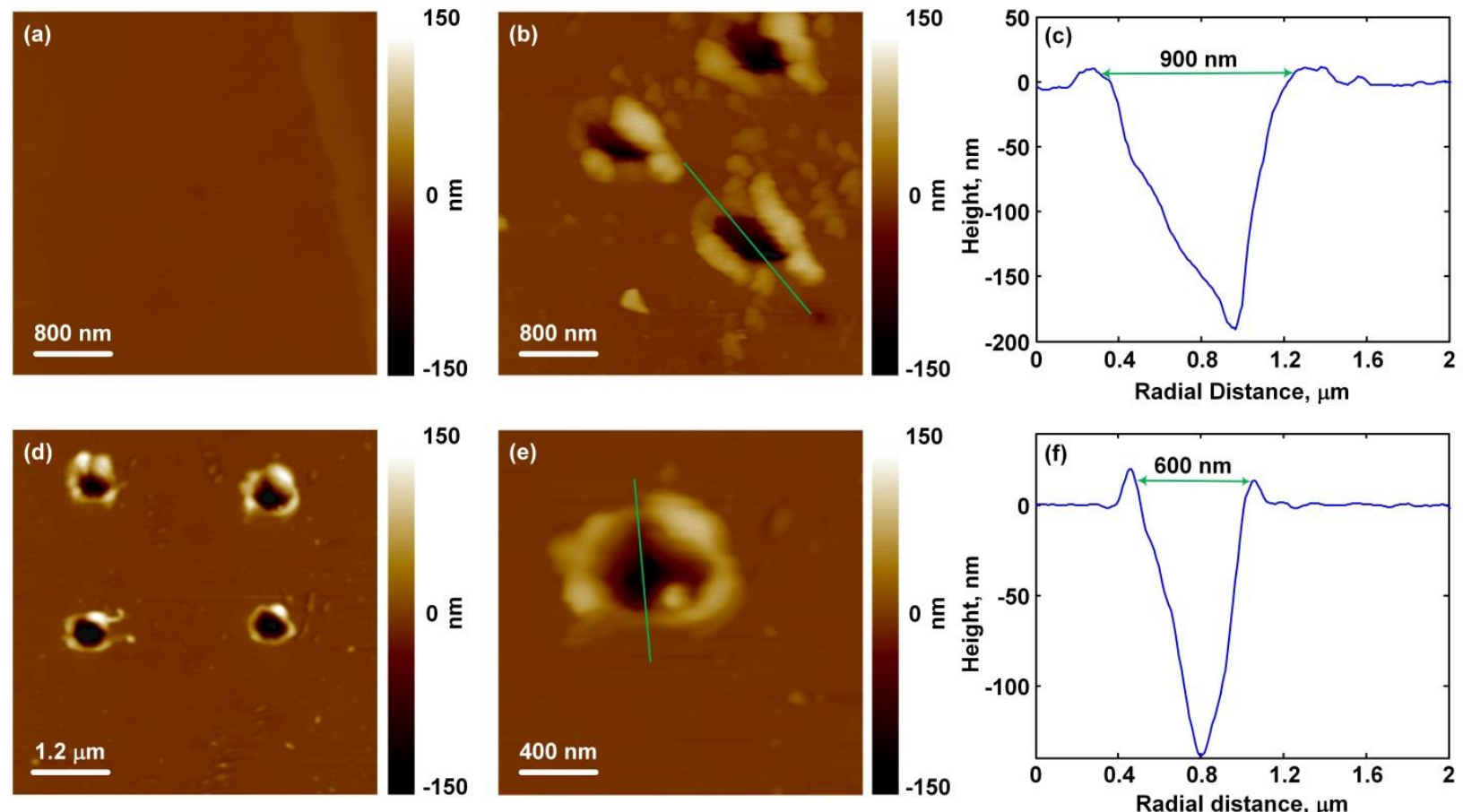

Figure 3-1. Near-field laser produced craters using AFM. (a) and (b) show AFM height images before and after the ablation experiment respectively with anthracene thin film; whereas the height images for ablation of rhodamine $6 \mathrm{G}$ are shown in (d) and (e). The depth profiles of the crater are shown in (c) and (f) for anthracene and rhodamine $6 \mathrm{G}$ respectively. 
and $100 \mathrm{~nm}$ wide; the smaller crater rim may be related to the higher melting point of rhodamine 6G $\left(290^{\circ} \mathrm{C}\right)$ compared to anthracene $\left(218^{\circ} \mathrm{C}\right)$ and will be affected by the film morphology and absorption of the two compounds.

The LDI mass spectrum of captured anthracene is shown in Figure 3-2. A total of 18 spots were irradiated under the conditions described above. Ablated anthracene from these spots was collected on a single flattened silver wire, which was cut to a length of $4 \mathrm{~mm}$ and mounted on a steel MALDI target with double-side conductive tape. Figure 3-2 a shows the mass spectrum of the silver wire alone. The peaks at $\mathrm{m} / \mathrm{z}$ of 107 and 109 arise from the abundant silver isotopes at approximately equal abundance. The peaks around $\mathrm{m} / \mathrm{z} 216$ and $\mathrm{m} / \mathrm{z} 324$ arise from $\mathrm{Ag}_{2}{ }^{+}$and $\mathrm{Ag}_{3}{ }^{+}$that are observed from direct irradiation of the silver surface, consistent with published results. ${ }^{238-239}$ Figure 3-2 b shows the LDI mass spectrum dipped in a $1 \mathrm{mg} / \mathrm{mL}$ solution of anthracene in acetonitrile solvent. The $\mathbf{M}^{+\bullet}$ peak for anthracene is observed at $m / z 178$. Figure 3-2 c shows the mass spectrum of anthracene near-field ablated and captured on the silver wire. Although the signal is two orders of magnitude lower, the anthracene peak is observed at $\mathrm{m} / \mathrm{z}$ 178.

Mass spectra of rhodamine 6G are shown in Figure 3-3. Figure 3-3 a shows a LDI mass spectrum obtained by dipping a silver wire in a $1 \mathrm{mg} / \mathrm{mL}$ dichloromethane solution of rhodamine. The prominent peaks at $\mathrm{m} / z 388,415$ and 444 are associated with rhodamine $6 \mathrm{G}$ LDI. The prompt fragmentation results from alkyl groups from the dye molecule and has been reported previously. ${ }^{240-241}$ Figure 3-3 b shows the LDI mass spectrum of rhodamine $6 \mathrm{G}$ obtained by transfer from 18 ablation spots under the conditions reported for Figure 3-1. The mass spectrum shows peaks at peaks at 388, 415 and 444 characteristic of rhodamine $6 \mathrm{G}$, although the 
388 peak is somewhat larger, suggesting that some fragmentation may have occurred during the ablation.
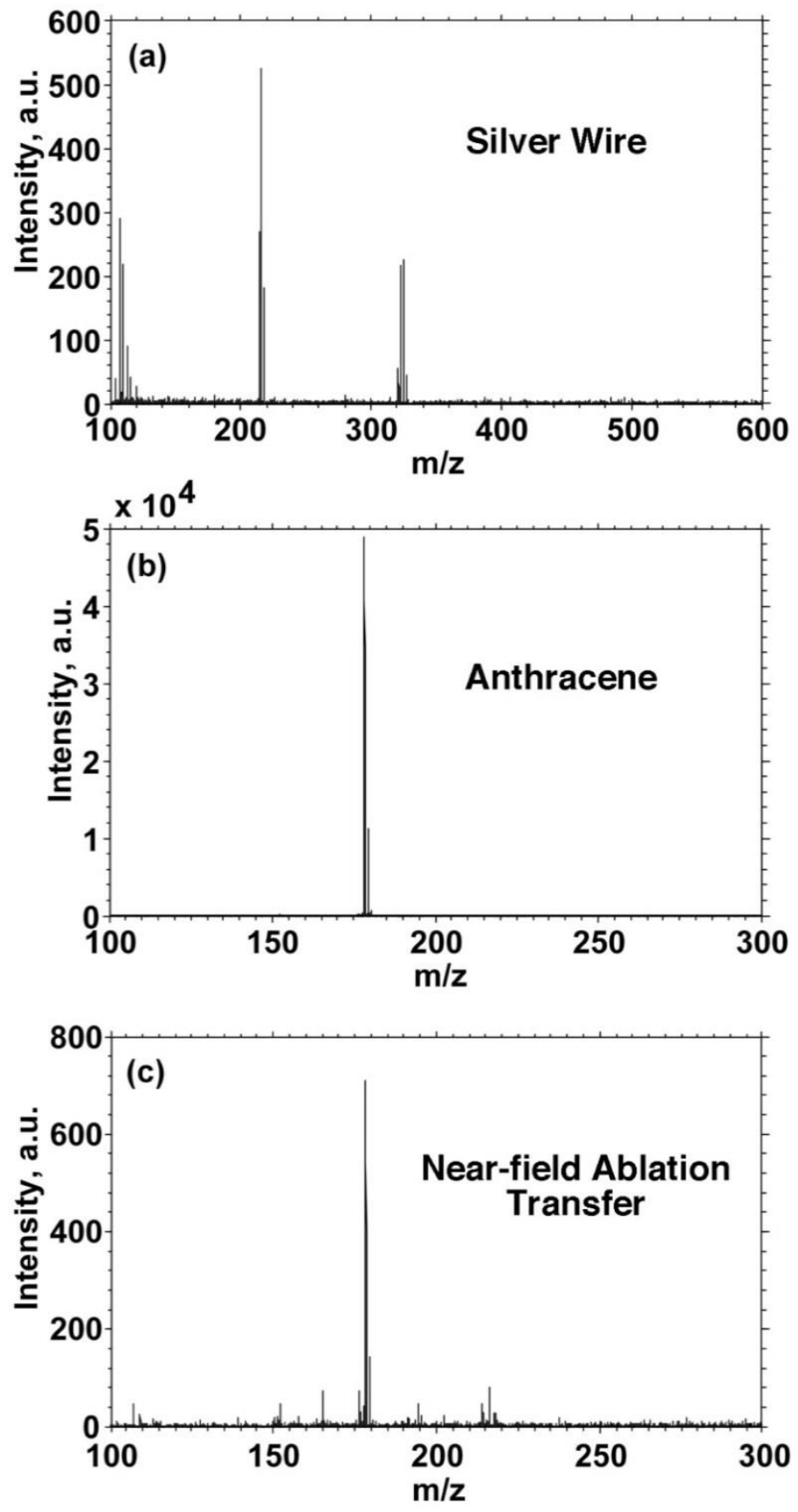

Figure 3-2. LDI mass spectra of (a) blank silver wire, (b) silver wire dipped in anthracene solution and (c) near-field laser ablation capture on silver wire. 
(a)

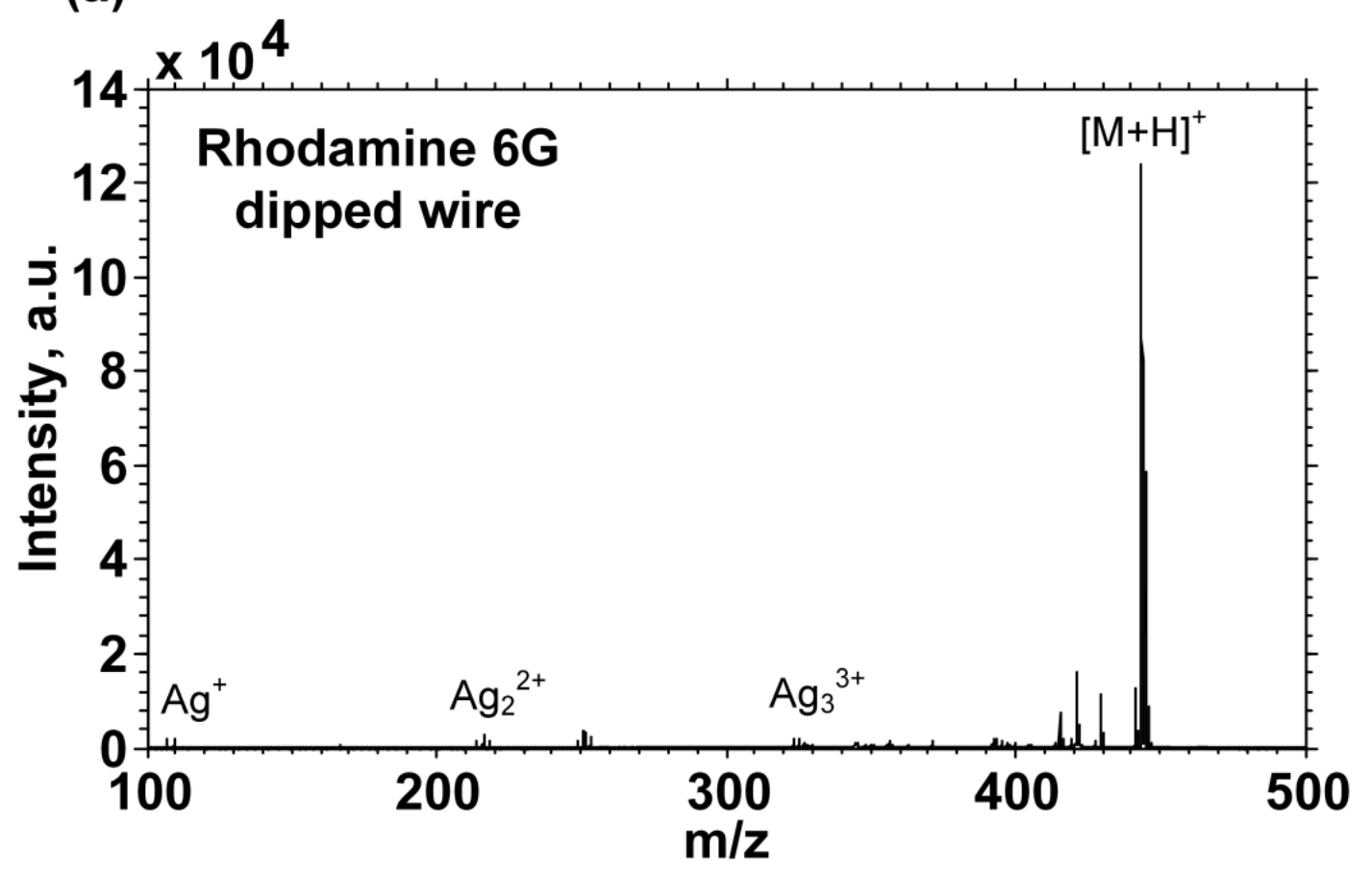

(b)

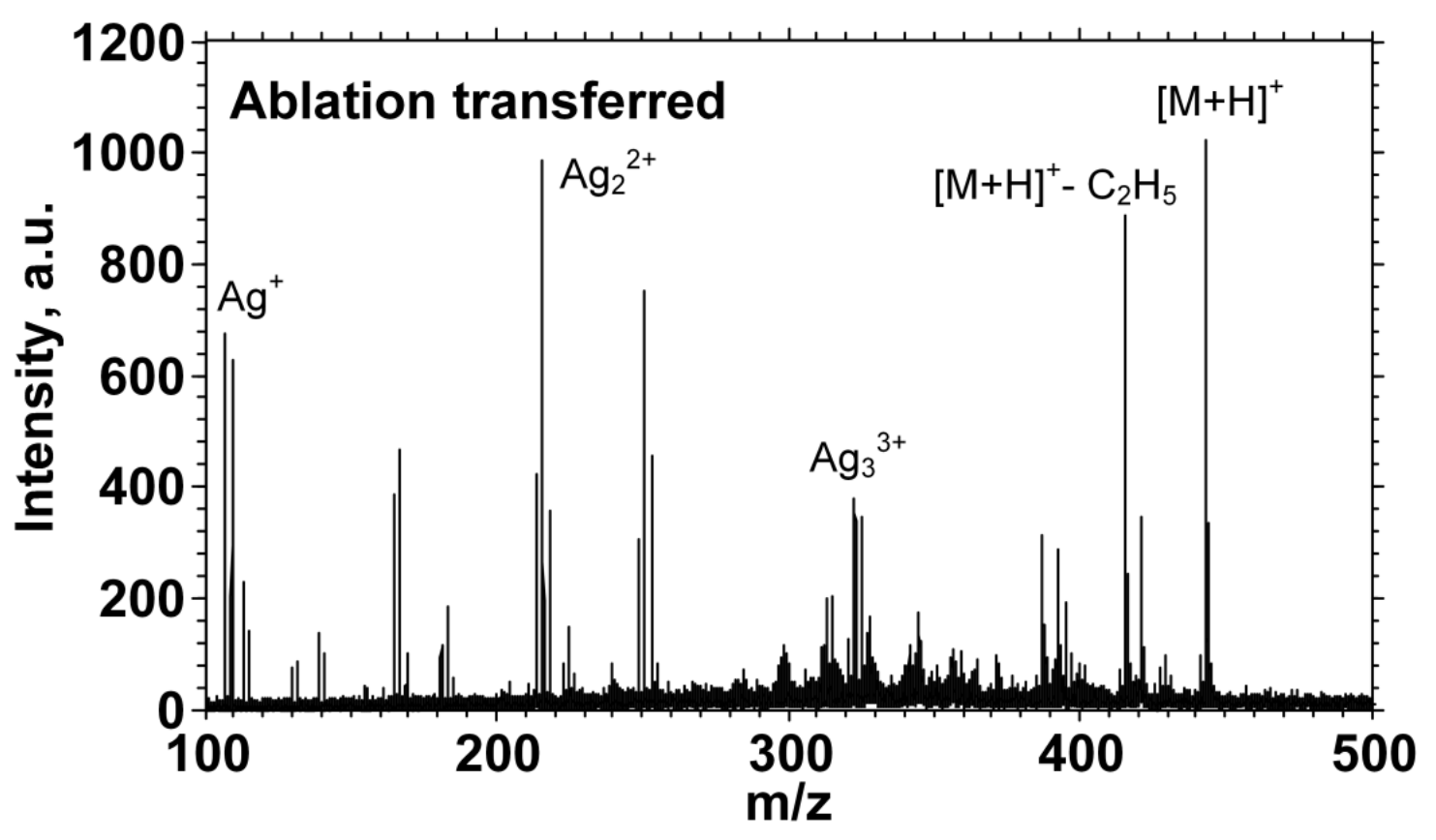

Figure 3-3. LDI mass spectra of (a) silver wire dipped in rhodamine 6G solution and (b) nearfield laser ablation transfer from 18 ablation spots to a silver wire. 
The efficiency of tip-enhanced laser assisted sample transfer was estimated by comparison of the LDI signal of transferred rhodamine $6 \mathrm{G}$ with the signal obtained from a sample deposited from a solution of known concentration. To test the transfer efficiency, a $5 \mu \mathrm{L}$ aliquot of rhodamine $6 \mathrm{G}$ from a $1 \mathrm{nM}$ solution in dichloromethane was deposited on the MALDI target to form an approximately $2 \mathrm{~mm}$ diameter spot containing $5 \mathrm{fmol}$ rhodamine 6G. LDI was performed exhaustively on the spot by irradiating each point on the deposit until the sample was depleted (several hundred laser shots) and the signal approached zero. The integrated signal for the base peak at $m / z 444$ was recorded and used as a calibration of signal per mol sample. The ablated rhodamine $6 \mathrm{G}$ spot dimensions were $900 \times 650 \mathrm{~nm}$ and $150 \mathrm{~nm}$ in depth, yielding 70 amol material for a single conical crater. Comparison of the signal obtained from multiple ablated craters (for example Figure 3-3 b) to the calibration gives a sample collection efficiency of $3 \%$ with an error of $0.5 \%$. This is similar to the efficiency reported for droplet collection using far-field ablation. ${ }^{176-177}$

We found that larger molecules could also be transferred without fragmentation using the tip-enhanced laser assisted sample transfer system. The extra steps of matrix addition and deposition were necessary to detect the larger molecules. Figure 3-4 shows the transfer of the peptide angiotensin II. Figure 3-4 a, is an AFM image of the laser-produced crater in a thin film of the peptide. The laser fluence was $1.1 \mathrm{~kJ} / \mathrm{m}^{2}$ and 30 shots were used to remove the material. The craters ranged from 500 to $800 \mathrm{~nm}$ deep with dimensions of $1100 \times 650$, corresponding to 500 amol of peptide. Assuming a 3\% transfer efficiency as determined above, this corresponds to 15 amol transferred to the wire. For the ablation of angiotensin II, the rim had a noticeable threefold rotational symmetry coincident with the edges of the triangular pyramid AFM tip, suggesting an edge enhancement of the electric field at these points (this can be observed to an 
extent in Figure 3-1, but is more pronounced here). The transferred material was dissolved in 5 $\mu \mathrm{L}$ of saturated CHCA in 1:1 acetonitrile and $0.1 \%$ TFA and deposited on the MALDI target for analysis.

(a)

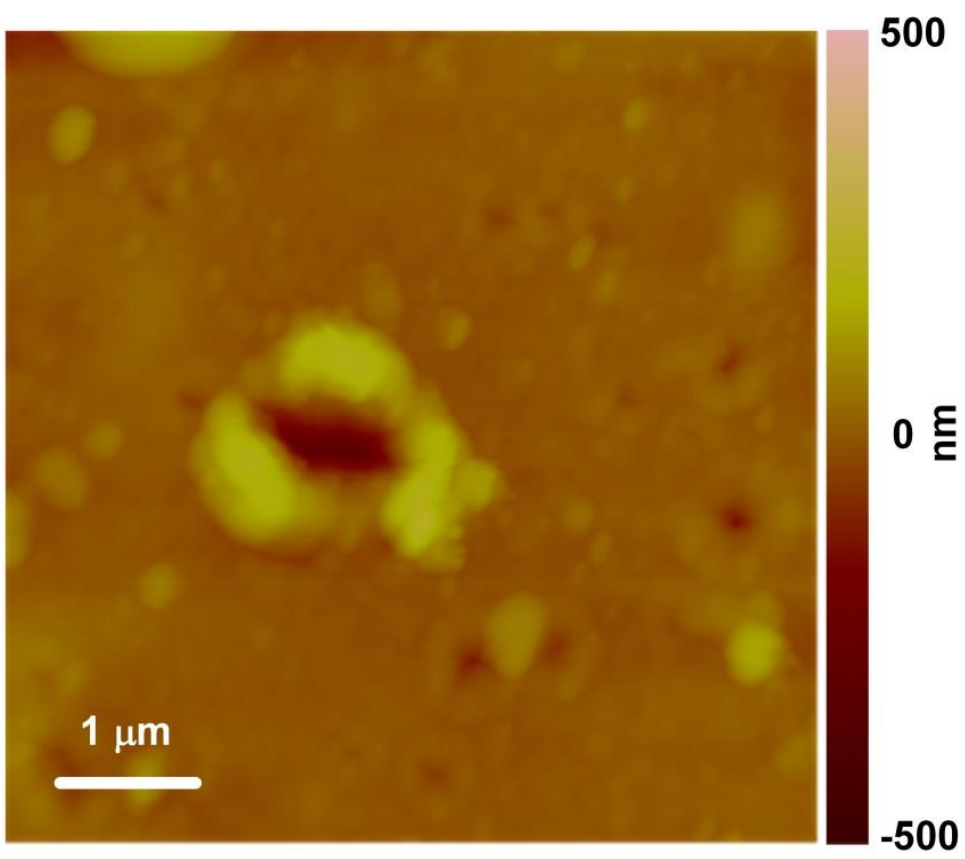

(b)

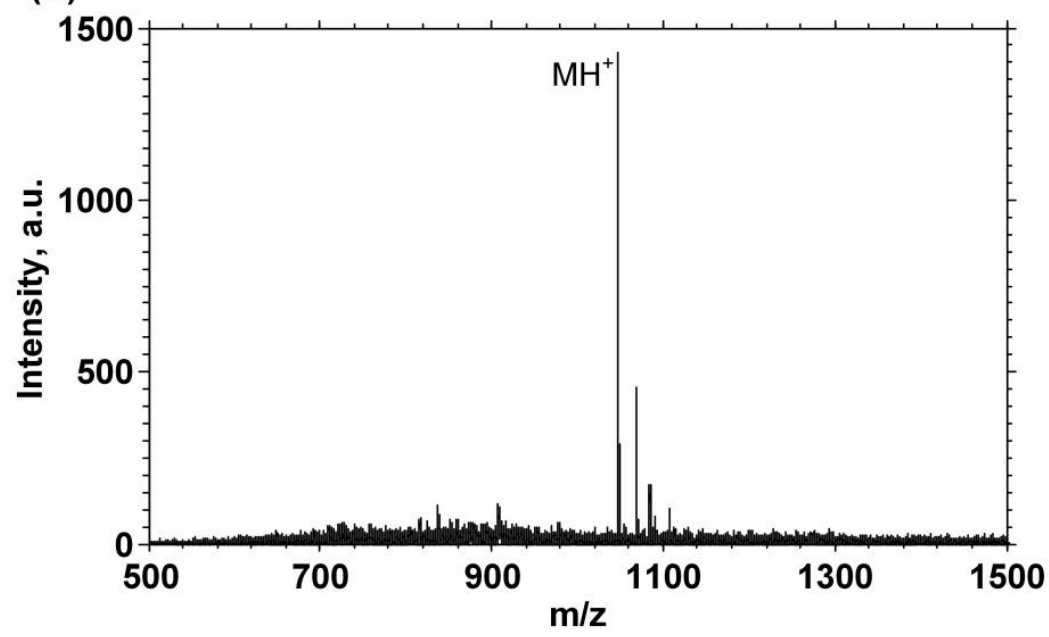

Figure 3-4. Tip-enhanced laser assisted sample transfer of the peptide angiotensin II showing a) AFM image of a laser produced crater and b) MALDI mass spectrum of material transferred from a single spot. 
The mass spectrum in Figure 3-4 b was obtained in positive ion reflectron mode. The most intense peak in the mass spectrum is protonated angiotensin $[\mathrm{M}+\mathrm{H}]^{+}$with sodium and potassium adduct peaks visible to higher mass.

The largest polypeptide that was transferred and observed by MALDI was bovine insulin. A linear mode MALDI mass spectrum of insulin is shown in Figure 3-5. The material was transferred at a laser fluence of $1.1 \mathrm{~kJ} / \mathrm{m}^{2}$ and 30 shots from ten individual spots, dissolved in matrix, and deposited on a MALDI target for analysis as above.

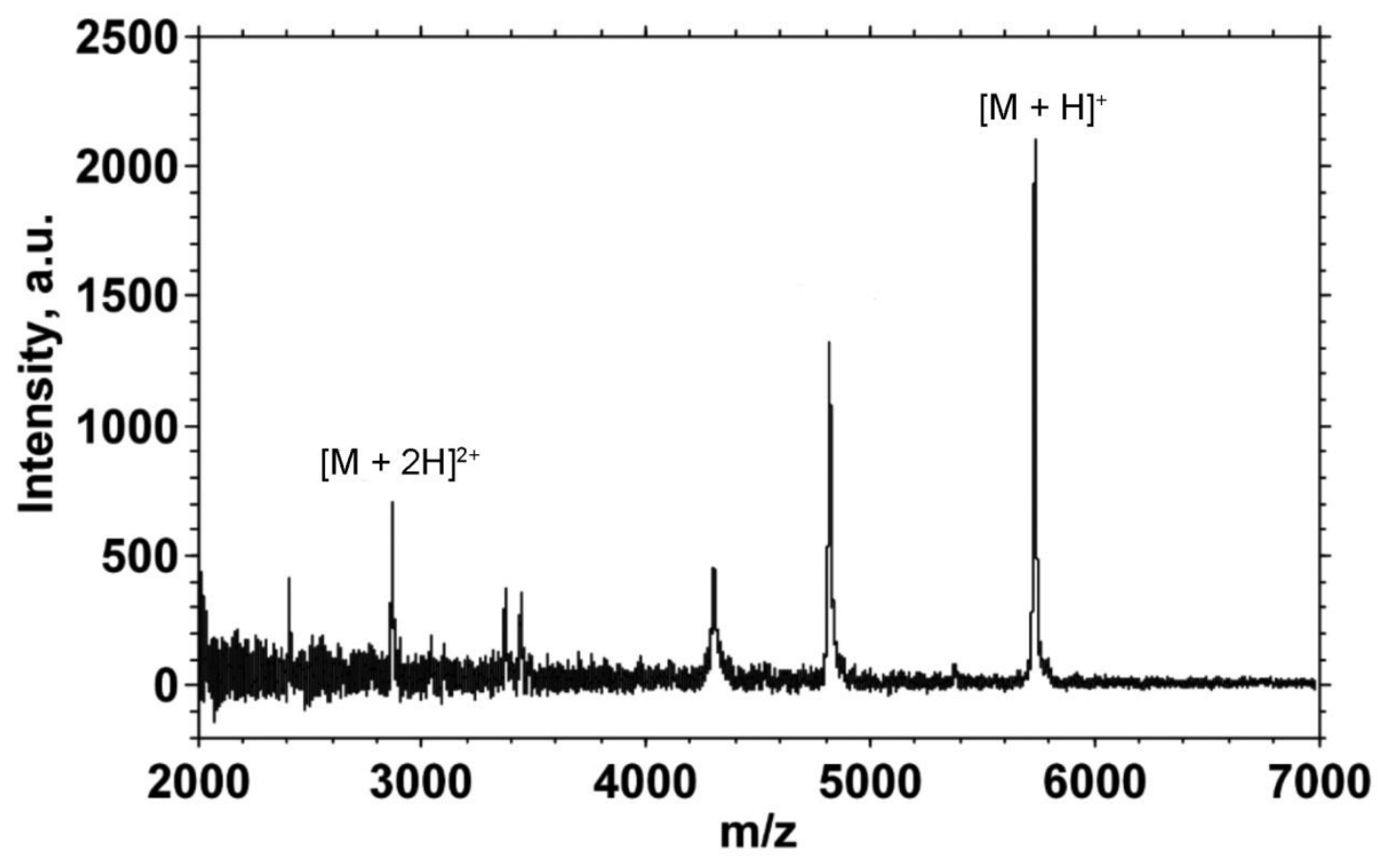

Figure 3-5. MALDI mass spectrum of bovine insulin transferred from 10 spots using tipenhanced laser assisted sample transfer. 
The base peak in the mass spectrum is protonated insulin with a doubly-protonated peak also observed. Several other peaks are observed, for example at $m / z, 4305$ and $m / z 4820$, and these are tentatively assigned to fragments of insulin. These peaks do not appear in MALDI spectra of an insulin standard obtained under comparable conditions and their intensity in relation to the insulin base peak varies from run to run. MALDI mass spectra of insulin standard obtained under comparable conditions do not contain fragments. The fragments were observed at the same $\mathrm{m} / \mathrm{z}$ in multiple spectra, although the relative peak intensities varied slightly from spectrum to spectrum. A similar procedure was used to transfer the proteins cytochrome c, lysozyme, and myoglobin. Although comparable craters were formed with these samples, no high mass signal was detected in linear MALDI mass spectra with either CHCA or sinapinic acid matrix.

The tip-enhanced material removal that we have observed may be a result of either local enhancement of the light field at the AFM tip or rapid heating of the tip with the thermal energy transferred to the sample. The observation that the optimum laser polarization is perpendicular to the tip axis rather than parallel is not consistent with a simple model of apertureless tip enhancement, ${ }^{67,233}$ but as noted above, the triangular pyramid shape of the AFM tip may lead to near-field enhancement at the pyramid edges with perpendicular polarization, similar to results from triangular nanoparticles. ${ }^{234-237}$ The fact that the ablation crater is much larger than the tip radius is somewhat unexpected, but is consistent with field enhancement at the pyramidal tip edges. The $600 \mathrm{~nm}$ to $1 \mu \mathrm{m}$ crater diameters are generally consistent with the crater sizes observed in previous studies using aperture ${ }^{242}$ and apertureless tips. ${ }^{218}$ The relative contributions of thermal and electric field effects remains an open question. ${ }^{179}$ 


\subsection{Summary}

Atomic force microscope tip-enhanced laser assisted sample transfer to a suspended silver wire was demonstrated with direct off-line LDI MS of the collected material on the wire and MALDI MS of the material dissolved in matrix and deposited on target. When the AFM tip in contact with the sample is irradiated with a pulsed $355 \mathrm{~nm}$ ns pulse width Nd:YAG laser, craters between $600 \mathrm{~nm}$ and $1 \mu \mathrm{m}$ in diameter and between 150 and $800 \mathrm{~nm}$ deep are formed. The ablation craters are approximately ten times larger than the AFM tip diameter; however, relatively large craters have been observed previously in near-field laser ablation. ${ }^{214,}{ }^{242}$ Some craters have a three-fold symmetrical ablation and melting pattern, suggestive of enhanced material removal at the edges of the triangular pyramid AFM tip. This result is consistent with the observation that laser polarization perpendicular to the tip axis is optimum for material removal through a near-field process. However, it is possible that the material removal is driven by AFM tip heating and rapid heat transfer to the sample. We note that we have observed analyte melting under conditions that also result in the formation of intact protonated analyte molecules. ${ }^{243}$

It is estimated that $3 \%$ of the material that is removed is redeposited on the suspended wire that was $300 \mu \mathrm{m}$ above the sample target. Previous studies have reported transport distances of $50 \mu \mathrm{m}$ or less for ablated material before it is deposited on the aperture probe. ${ }^{214}$ Other studies

have shown that jets of particulate are ejected to distances of more than $100 \mu \mathrm{m} .{ }^{242}$ It is possible that the angled AFM tip does not significantly obstruct the ablation plume so that a sufficient amount of material is transported to the capture wire. Alternatively, the wire may simply be capturing that fraction of the material that does not condense into particulate. 
The largest molecule that was transferred intact was bovine insulin at 5733 molecular mass. Some specific fragmentation was observed in the insulin mass spectrum that is most likely the result of the laser ablation sample transfer process. It was possible to ablate larger proteins, but these were not detected either due to fragmentation or insufficient sensitivity. Ongoing work is being directed at improving the amount of material collected and capturing material from cells and tissue. Because the capture is separate from analysis, tip-enhanced capture of materials can be used with analysis methods other than mass spectrometry such as DNA/RNA sequencing and fluorescence assays. 


\section{CHAPTER 4. TIP-ENHANCED LASER ABLATION SAMPLE TRANSFER FOR LARGE BIOMOLECULE AND TISSUE MASS SPECTROMETRY*}

The goal of the research described in this chapter was to utilize tip-enhanced laser ablation (TELA) system to ablate intact biomolecules followed by MS detection. Atomic force microscope (AFM) TELA was used to transfer biomolecules from thin films and tissue sections to a suspended silver wire for off-line mass spectrometry using matrix-assisted laser desorption ionization (MALDI). In this work, the ability of coupling visible and near IR laser radiation with the TELA capture approach to ablate biomolecules was also tested. The fundamental wavelength of the Nd:YAG laser at $1064 \mathrm{~nm}$, and $532 \mathrm{~nm}$ wavelengths were used to ablate tissue sections and thin films of peptides and protein samples respectively. The ability of these wavelengths to transfer biomolecules without fragmentation was tested and the effect of laser polarization on the efficiency of material removal was also studied. ${ }^{244}$

\subsection{Introduction}

As discussed in Chapter 1, the resolution of laser-based mass spectrometry imaging is constrained by the diffraction limited laser spot size, which is approximately $200 \mathrm{~nm}$, half of the wavelength a UV laser. The best resolution is obtained in transmission mode where the laser irradiates the back side of the sample to eject material from the front side. An objective lens can focus the laser to a spot size below $1 \mu \mathrm{m},{ }^{61}$ but the drawback to transmission mode is that it requires extremely thin samples of $1 \mu \mathrm{m}$ thickness or less. In reflection mode, a standard high numerical aperture objective cannot be used because the low working distance means that the

\footnotetext{
* Some portions of the work reported in this chapter was originally published as Murray, K. K.; Ghorai, S.; Seneviratne, C. A., Tip-Enhanced Laser Ablation Sample Transfer for Mass Spectrometry, and has been published in MRS Proceedings. ${ }^{244}$ Reprinted with permission from the Cambridge university press.
} 
ablated material or ions are blocked by the objective. A unique solution to this problem is an objective lens with a central hole through which the desorbed ions pass. This approach has been used to produce focused spots of $600 \mathrm{~nm}$ and imaging resolution of $1 \mu \mathrm{m}$ under vacuum ${ }^{96}$ and a $25 \mu \mathrm{m}$ spot for atmospheric pressure MALDI. ${ }^{245}$ Long working distance Schwarzschild objectives have been used for laser desorption ionization imaging at $1 \mu \mathrm{m}$ lateral resolution with at a working distance more than $10 \mathrm{~cm} \cdot .^{77-78}$

As discussed in Chapter 1, there are two laser-based mass spectrometry imaging modes that are not limited by the ability to focus laser. The first of these is microscope imaging mode in which ions are created from a large spot but are mapped to a two-dimensional detector at high resolution. This approach has been used to obtain $4 \mu \mathrm{m}$ lateral resolution with a $200 \mu \mathrm{m}$ laser spot, ${ }^{21}$ which is below the diffraction limit for the IR laser used. ${ }^{22,}{ }^{94} \mathrm{~A}$ second approach is nearfield desorption and ablation: aperture near-field uses a reflective-coated optical fiber probe and apertureless near-field uses a sharp conductive tip. ${ }^{94}$ Direct ionization by laser desorption ionization has been accomplished by aperture near-field in vacuum ${ }^{210}$ or under ambient conditions with the ions directed into the mass spectrometer. ${ }^{224}$ Electron ionization has been used in conjunction with ambient aperture near-field ablation where molecules desorbed at atmospheric pressure were sampled into vacuum through a capillary tube and directed into the ion source. ${ }^{212-213}$ Apertureless near-field ablated material has been sampled into inductively coupled plasma mass spectrometry (LA-ICP-MS) with sub-micrometer lateral resolution. ${ }^{218}$

In the work described in this Chapter, we have extended the UV TELA capture approach to visible and near IR wavelengths. Experiments were performed with a pulsed nanosecond Nd:YAG laser fundamental $(1064 \mathrm{~nm})$, and doubled $(532 \mathrm{~nm})$ wavelengths to ablate rat brain tissue sections and peptide and protein samples from deposited thin films respectively. The 
material was collected, mixed with a matrix, and ionized in a MALDI time-of-flight mass spectrometer.

\subsection{Experimental}

The AFM laser ablation sample transfer configuration described in Chapter 2 was used in all experiments reported in this section. Standard proteins and peptides were deposited from solution onto a glass coverslip mounted on a circular metal disc. Typically, a $50 \mu \mathrm{L}$ volume of solution was deposited and the film was formed by slow evaporation. In this work, insulin and a peptide mixture were studied. The mixture of peptides was prepared by mixing solutions of bradykinin fragments (1-7), angiotensin II, angiotensin I, and renin substrate tetradecapeptide porcine in 3:4:5:6 molar ratio. It was possible to form thin films of insulin, however, the peptide mixture tended to segregate into separate crystals after slow evaporation. For tissue analysis, 10 $\mu \mathrm{m}$ thick mouse brain tissue sections that were thaw-mounted on $25 \mathrm{~mm} \times 50 \mathrm{~mm}$ glass microscope slide were mounted on a circular metal disc. The AFM images of the sample surface were obtained in tapping mode. All topographic images were obtained using the same experimental conditions.

The ablation wavelength was $532 \mathrm{~nm}$ or $1064 \mathrm{~nm}$ pulsed nanosecond laser. The laser was focused using a $25 \mathrm{~cm} \mathrm{CaF}_{2}$ lens to a spot size of approximately $600 \mu \mathrm{m}$ and was attenuated using a variable neutral density filter. The polarization of the beam was adjusted with a rotating half-wave plate. Ablation was accomplished by irradiating the AFM tip with the mildly focused laser as described in Chapter 2.

All the samples were prepared according to the procedures described in Chapter 2 and the captured material was analyzed by MALDI instrument. To enhance the MALDI signal, exhaustive sampling of the deposit was performed and the sum of the spectra is reported. 


\subsection{Results}

First, the capability of ablating material using $532 \mathrm{~nm}$ wavelength was tested. The working conditions were optimized for $532 \mathrm{~nm}$ ablation of peptides and proteins. The first sample analyzed was a peptide mixture created by mixing four peptide solutions. Figure 4-1 a, shows the sample region scanned prior to ablation. The ablation was performed with a single laser shot at every ablation spot with a laser fluence of $1 \mathrm{~kJ} / \mathrm{m}^{2}$. After selective ablation of material removal from the top half of the targeted sample region, the sample appeared as shown in Figure 4-1 b. Additional material was selectively ablated and captured and the resulting images are shown in Figure 4-1 c and 4-1 d. The ablated materials are captured on the wire and analyzed by MALDI. The captured material was dissolved in $5 \mu \mathrm{L}$ of CHCA matrix solution by extensive washing of the tip of the wire. Then, the solution was deposited on the MALDI target for analysis. Figure 4-2 shows the mass spectrum of the ablated and captured peptide mixture. We were able to detect all four peptides in the mixture. As shown in Figure 4-2, peaks at $m / z$ of 757, 1046.6, 1296.6 and 1759.1 were observed for bradykinin fragments (1-7), angiotensin II, angiotensin I and renin substrate, respectively. Due to the low sample quantity, the spectrum was obtained by exhaustive sampling of the MALDI target spot. The intensities of the peptide peaks do not match the peptide concentration in solution, particularly for bradykinin (1-7), which has a low signal. This is most likely due to separation of the peptides on the target prior to near-field ablation and capture. 

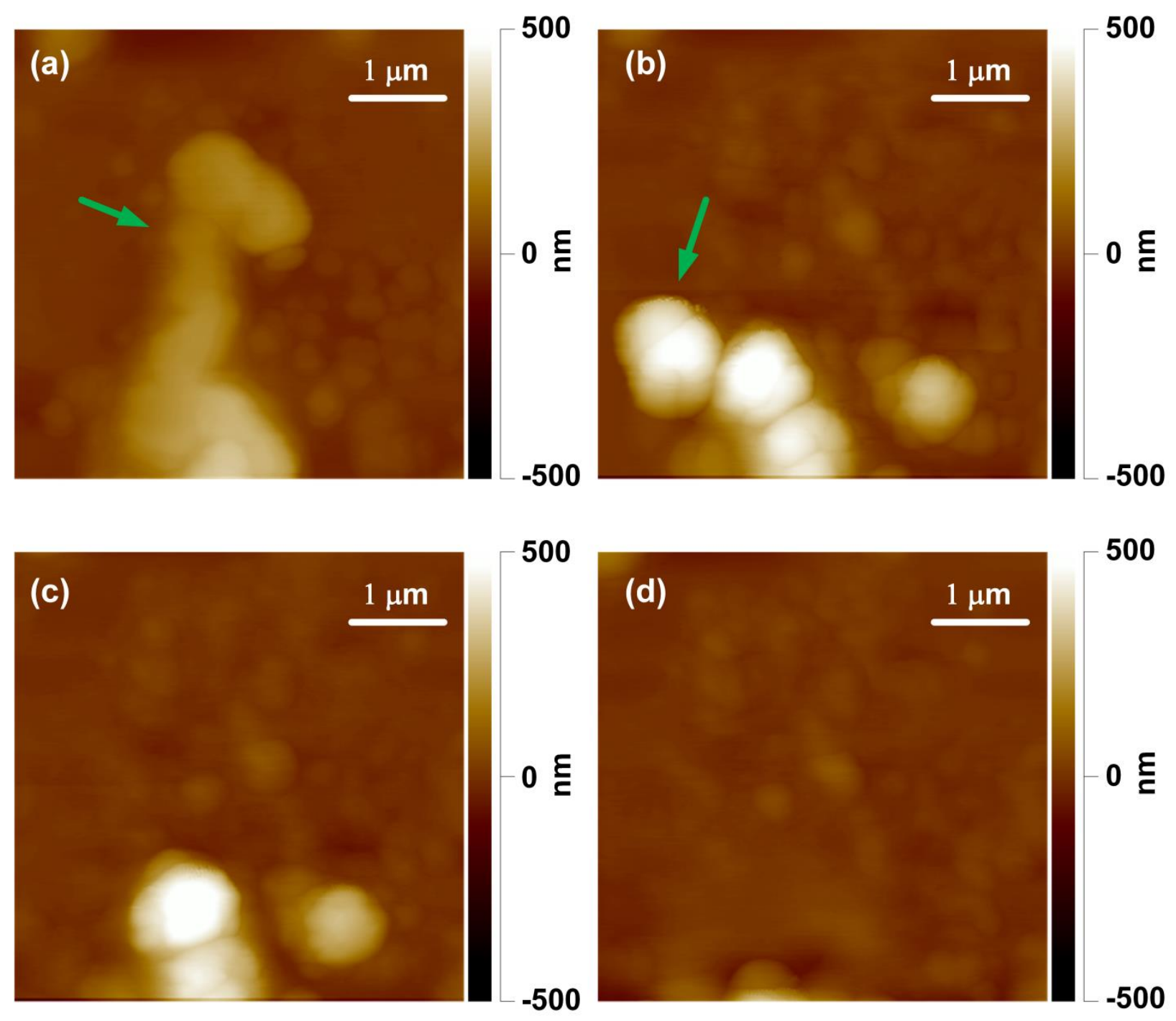

Figure 4-1. AFM images of (a) $5 \mu \mathrm{m} \times 5 \mu \mathrm{m}$ region of a deposited peptide mixture; the light region indicated by the green arrow corresponds to the sample; (b) material removal is observed after ablation from the top half of the sample and the green arrow indicates the material targeted for ablation; (c) removal of some of the remaining material; (d) sample region after complete material removal. 


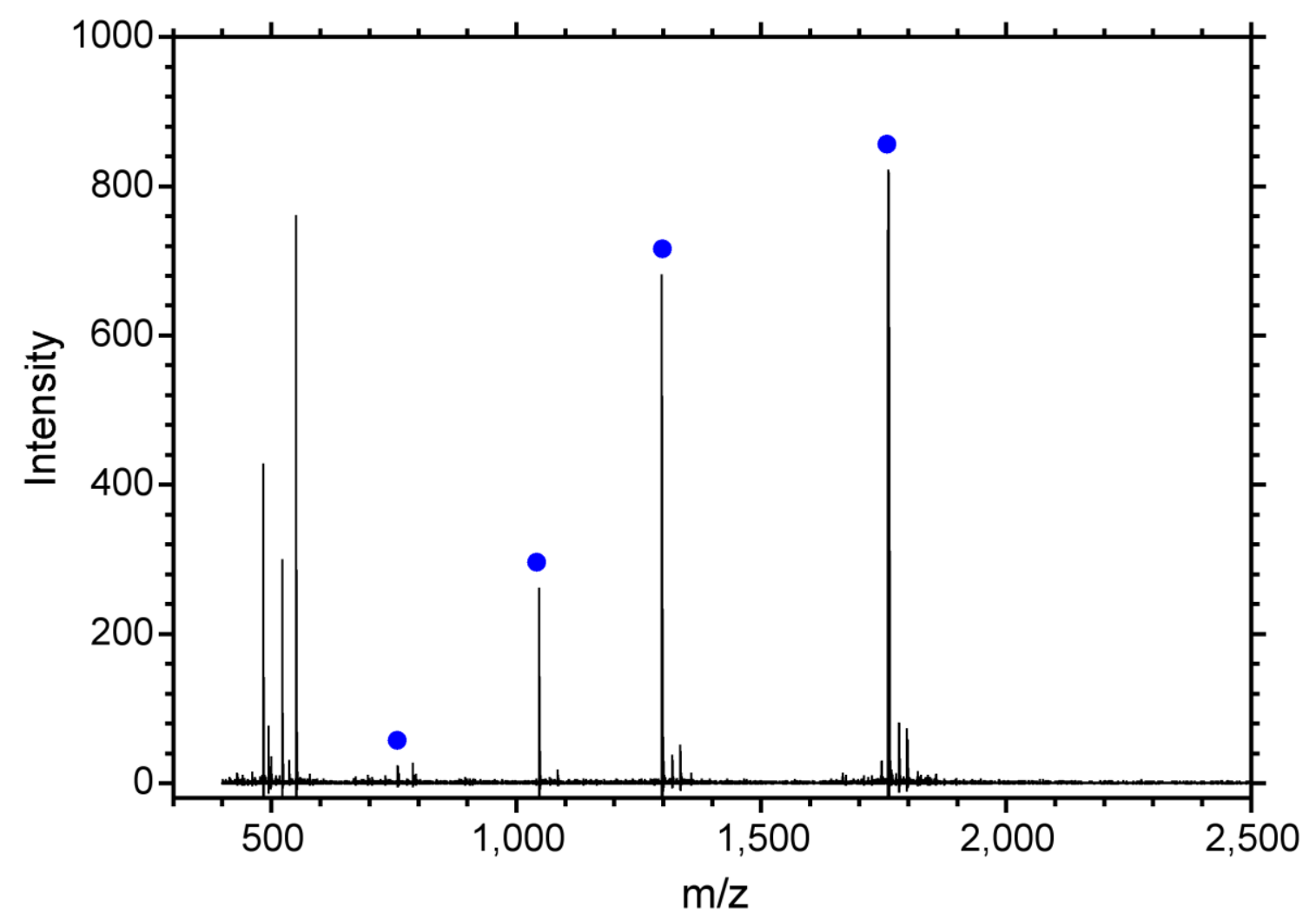

Figure 4-2. Mass spectrum of a peptide mixture ablated and captured for MALDI analysis; the blue circles indicate the protonated peptide peaks.

A topographical image of insulin ablation crater and a MALDI mass spectrum of insulin obtained using $532 \mathrm{~nm}$ tip-enhanced laser ablation transfer are shown in Figures 4-3 and 4-4 respectively. The protein was transferred from 10 individual ablation spots, dissolved in matrix, and deposited on the MALDI target for analysis. The most intense peak in the mass spectrum is the protonated insulin molecule at $\mathrm{m} / \mathrm{z}, 5734$. The doubly protonated insulin molecule is also observed at $m / z, 2868$. In contrast to laser ablation transfer at $355 \mathrm{~nm},{ }^{231}$ additional fragmentation peaks are not observed. The gentler material transfer at $532 \mathrm{~nm}$ as compared to $355 \mathrm{~nm}$ may be due to the stronger plasmon absorption expected at this wavelength for the $30 \mathrm{~nm}$ gold coated AFM tip. ${ }^{180}$ 


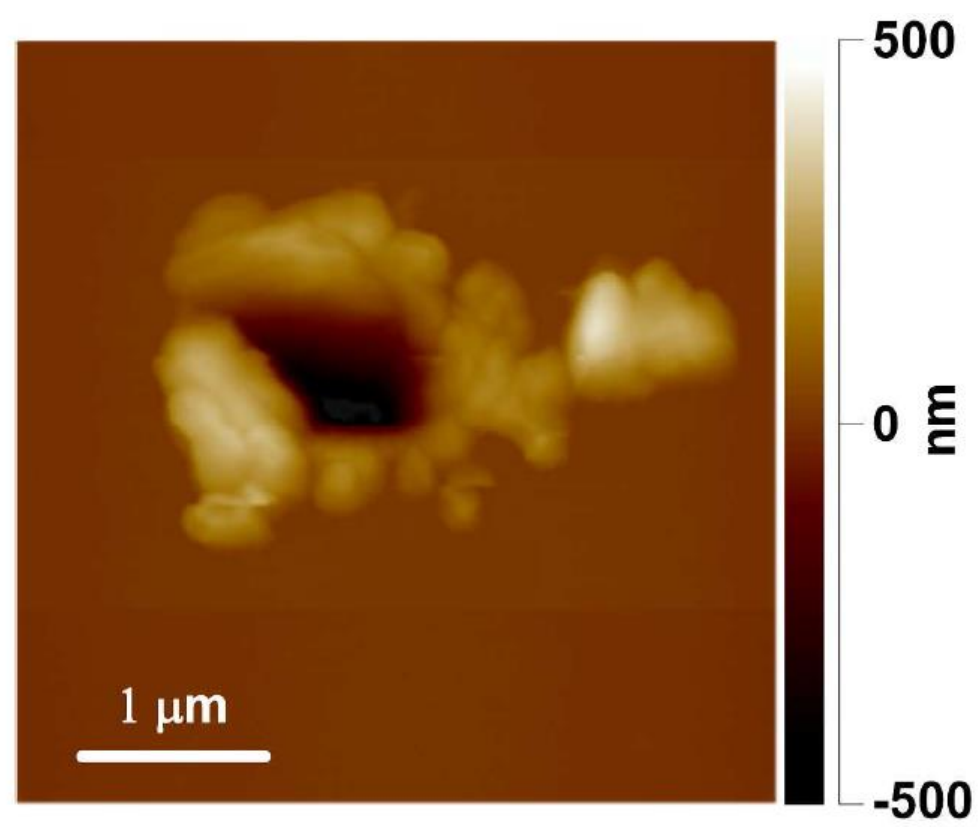

Figure 4-3. Insulin ablation crater from $532 \mathrm{~nm}$ tip-enhanced laser ablation.

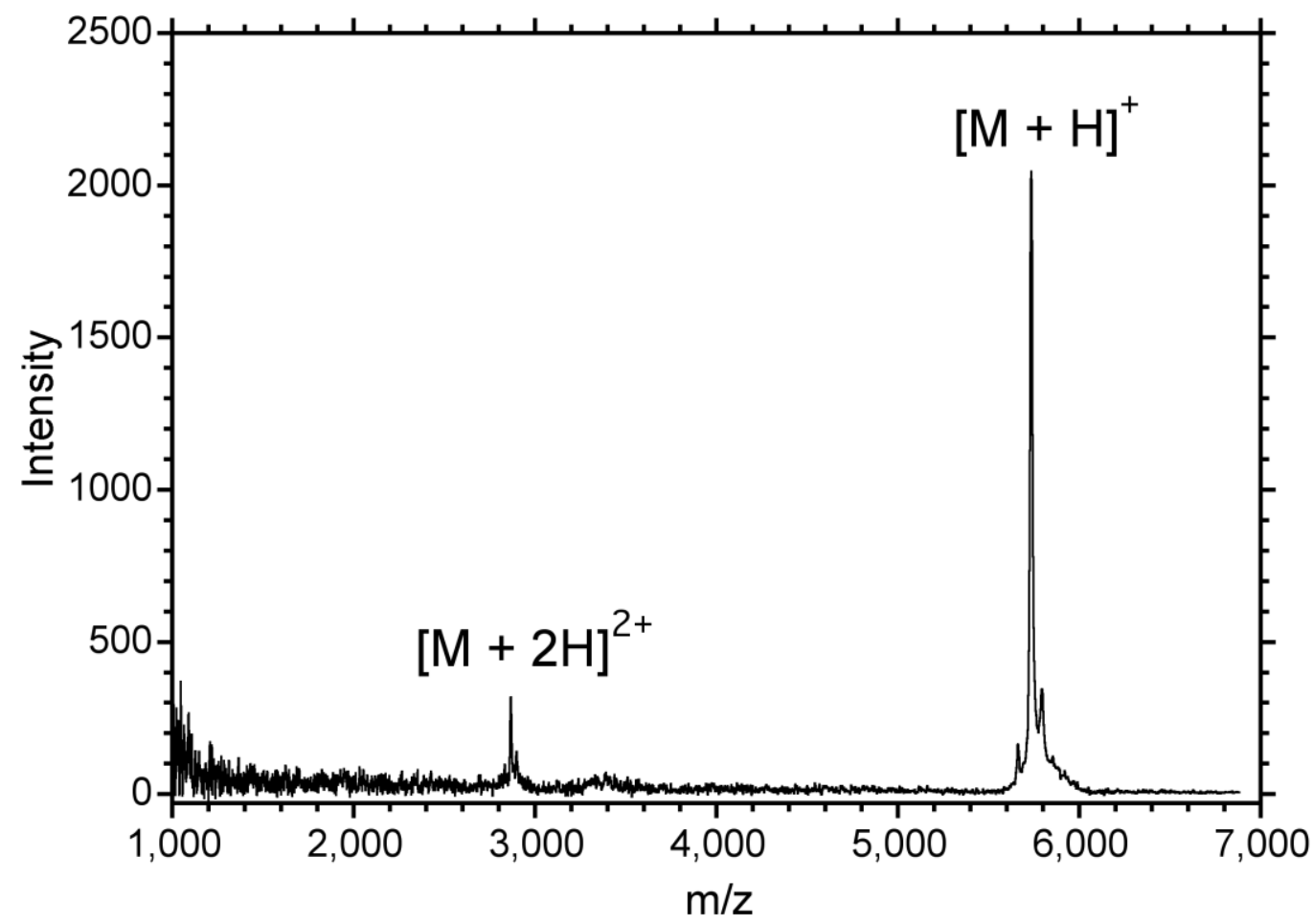

Figure 4-4. MALDI mass spectrum of the protein insulin using AFM tip-enhanced ablation and capture. 
One of the most significant differences between $532 \mathrm{~nm}$ near-field laser ablation and 355 $\mathrm{nm}$ ablation is the laser polarization dependence. At $355 \mathrm{~nm}$ it was found that s-polarization with the laser electric field perpendicular to the tip axis gave the best ablation. ${ }^{231}$ The optimum polarization for apertureless near-field ablation is parallel to the optical antenna axis. ${ }^{180}$ With 532 $\mathrm{nm}$ radiation, p-polarization is favored and removes more than five times as much material as spolarization and is consistent with a near-field effect.

Next, we have utilized the TELA approach to capture material from sub-micrometer size craters on biological tissue samples. The $1064 \mathrm{~nm}$ wavelength was used for tissue ablation with the rationale that the near-IR absorption window of the tissue will be minimally disruptive. The ablated material was collected, mixed with a matrix and analyzed in the MALDI time-of-flight mass spectrometer. Removal of material with micrometer sized ablation craters, AFM topographical images of the ablation craters on the rat brain tissue, and MALDI detection of the captured material were demonstrated.

The working conditions of the TELA system such as laser energy and focus were adjusted for the tissue ablation using $1064 \mathrm{~nm} \mathrm{Nd-YAG} \mathrm{laser} \mathrm{to} \mathrm{obtain} \mathrm{craters} \mathrm{on} \mathrm{rat} \mathrm{brain} \mathrm{tissue.} \mathrm{First,}$ the rat brain tissue section $(10 \mu \mathrm{m}$ thickness) slide was placed on the AFM stage and observed from the AFM video camera for the selection of the area of interest. Figure 4-5 a, shows the AFM height image of the sample region scanned prior to ablation. The ablation was performed by placing the AFM probe $15 \mathrm{~nm}$ above the surface of the rat brain tissue and irradiating for 3 seconds with a repetition rate of $10 \mathrm{~Hz}$ at a laser fluence of $12 \mathrm{~kJ} / \mathrm{m}^{2}$. The resulting crater is shown in Figure 4-5 b. The depth profile of the crater along the cursor line indicated in Figure 4$5 \mathrm{~b}$ is shown in Figure 4-5 c. The average depth of the ablation craters from three replicates 
under the conditions used for Fig. $4-5 \mathrm{~b}$ was $400 \pm 30 \mathrm{~nm}$ and the average ablation spot area was $9.5 \pm 0.8 \mu \mathrm{m}^{2}$.
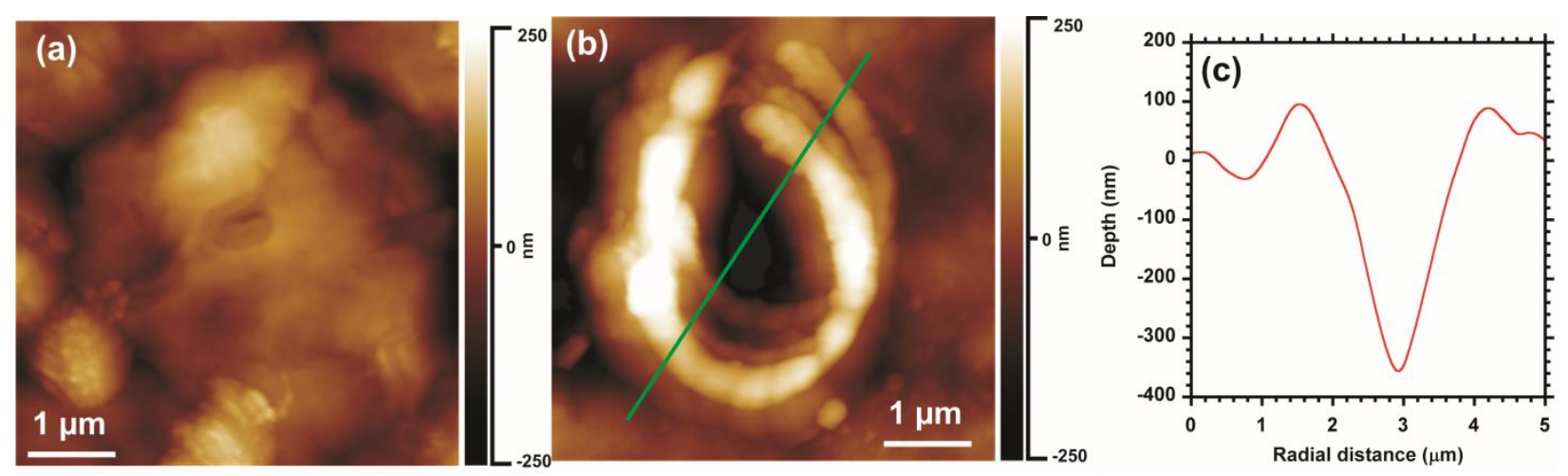

Figure 4-5. TELA produced crater on rat brain tissue using AFM. (a) and (b) show AFM height images before and after the ablation experiment respectively with rat brain tissue section. The depth profile of the crater is shown in (c) for rat brain tissue section.

The ablated material was captured and analyzed by MALDI according to the previously reported protocol. ${ }^{231}$ The resulting mass spectrum is shown in Figure 4-6 a. In this spectrum the most intense peaks in the $\mathrm{m} / \mathrm{z}$ range between 400 and 600 are from the CHCA matrix ions (indicated with asterisks). A magnified view of the spectrum in the $m / z$ range between 600 and 1100 is shown as an inset where phospholipids are typically detected. ${ }^{178,246}$ No peaks were observed above $m / z 1100$ for any of the tissue samples. The dominant mass peaks in Figure 4-6 between $\mathrm{m} / \mathrm{z} 600$ and 1100 are assigned to phosphatidylcholines (PCs). In this spectrum, prominent ions at $m / z$ 734.6 $[\mathrm{PC}(32: 0)+\mathrm{H}]^{+}, 760.6[\mathrm{PC}(34: 1)+\mathrm{H}]^{+}$, and $788.6[\mathrm{PC}(36: 1)+\mathrm{H}]^{+}$ are labelled. Here, PC indicates phosphatidylcholine, and the numbers in parentheses indicate the alkyl chain length and number of double bonds, respectively. 


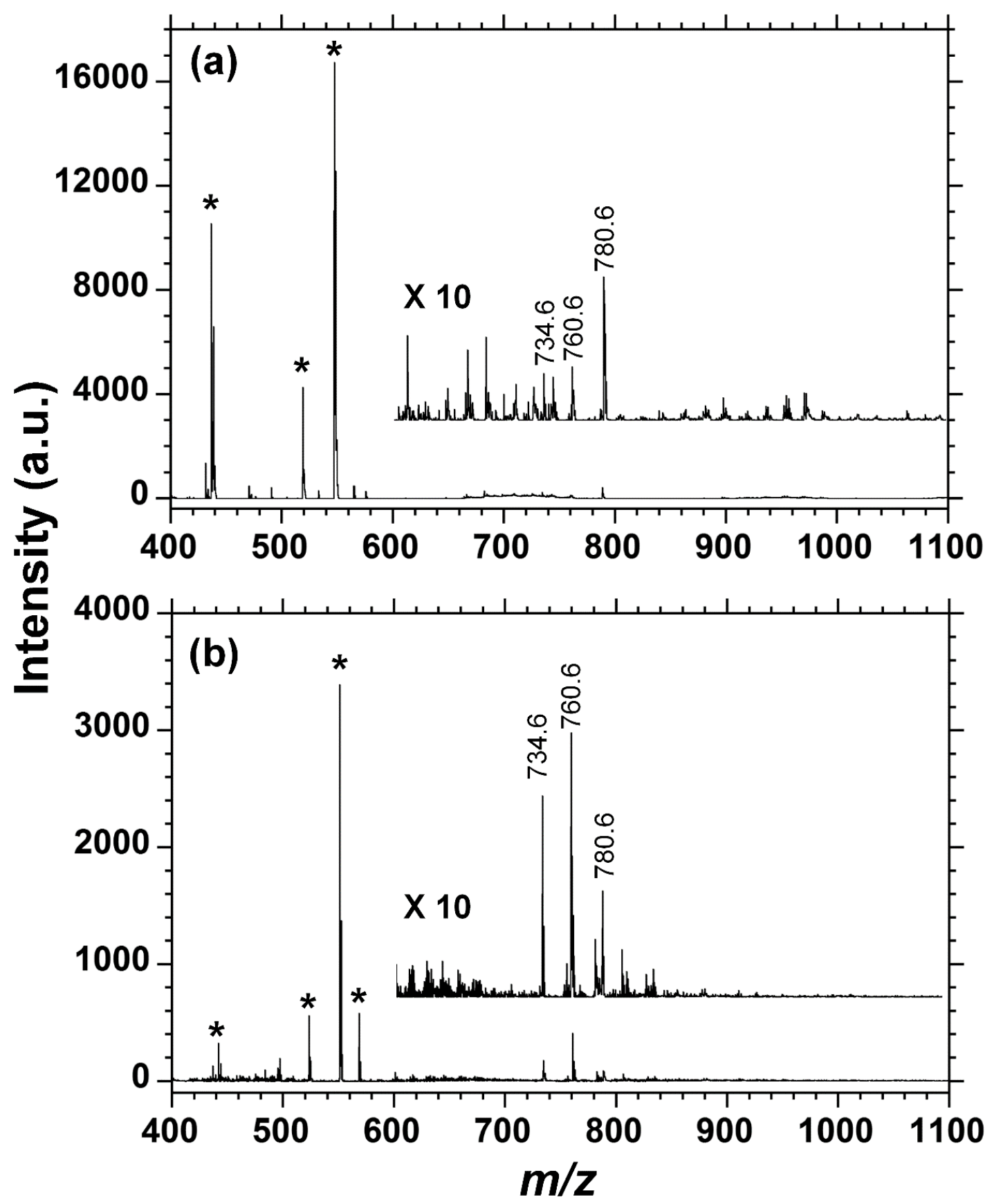

Figure 4-6. MALDI mass spectra from rat brain tissue sample (a) solvent extracted rat brain tissue sample (b) from a TELA produced crater shown in Figure 4-5 b. A magnified view of the $\mathrm{m} / \mathrm{z}$ 600-1100 region is shown in the inset for each spectrum. In this spectrum, prominent ions at $m / z 734.6[\mathrm{PC}(32: 0)+\mathrm{H}]^{+}, 760.6[\mathrm{PC}(34: 1)+\mathrm{H}]^{+}$, and $788.6[\mathrm{PC}(36: 1)+\mathrm{H}]^{+}$are shown. $\mathrm{PC}$ indicates phosphatidylcholine, and the numbers in parentheses indicate the alkyl chain length and number of double bonds, respectively. 
In order to compare with the detected material sampled by TELA, a MALDI mass spectrum was obtained from a portion of the same tissue section of the rat brain tissue sample that was used for TELA experiments. For this experiment, the tissue sample was treated according to the Folch extraction procedure and mixed with $2 \mu \mathrm{L}$ of CHCA matrix solution and the solution was then deposited on the MALDI target for analysis. Mass spectra are shown in Figure 4-6 b. The resulting mass spectrum from solvent extracted MALDI analysis of rat brain tissue is comparable with the TELA MALDI MS analysis on rat brain tissue.

MALDI mass spectra from two different regions on the tissue section were also obtained using TELA. The resulting mass spectra are shown in Figure 4-7. In these experiments, the AFM tip was moved to a different region on the rat brain tissue section and ablated for three seconds using the same laser conditions as described in the previous section. The captured material was dissolved in matrix and deposited on the MALDI target for analysis. Figures 4-7 a and 4-7 b show mass spectra obtained from the first ablation spot and the second ablation spot in the $\mathrm{m} / \mathrm{z}$ range between 700 and 900 . The first ablation spot showed prominent ions at $\mathrm{m} / \mathrm{z} 734.6$ and 788.6 while the second spot (Figure 4-7b) showed only $\mathrm{m} / \mathrm{z}$ 734.6. However, $\mathrm{m} / \mathrm{z} 760.6$ $[\mathrm{PC}(34: 1)+\mathrm{H}]^{+}$peak was absent from both of these spots. This indicates the irreproducibility inherent to MALDI analysis or uneven distribution of PC's in rat brain tissue.

Several studies have shown that phosphatidylcholines (PC), phosphatidylethanolamines (PE), and sphingomyelins (SM), can be identified by direct tissue analysis using MALDI-TOFMS. ${ }^{247-248}$ Similar PC's were also identified by IR laser ablation sample transfer using MALDITOF-MS for rat brain tissue analysis. ${ }^{178}$ Furthermore, it has been reported that peaks associated with PC's tend to dominate the mass spectrum, since phospholipids comprise more than $50 \%$ of membrane phospholipids in eukaryotic organisms. ${ }^{249-250}$ It is important to note that the MALDI 
spectra observed from TELA experiments correspond to craters with an average area of $9 \mu \mathrm{m}^{2}$ and the average depth of $400 \mathrm{~nm}$ which corresponds to approximately $4 \mathrm{pg}$ of material, assuming the density of rat brain tissue is $1.02 \mathrm{pg} / \mu \mathrm{m}^{3}{ }^{251}$

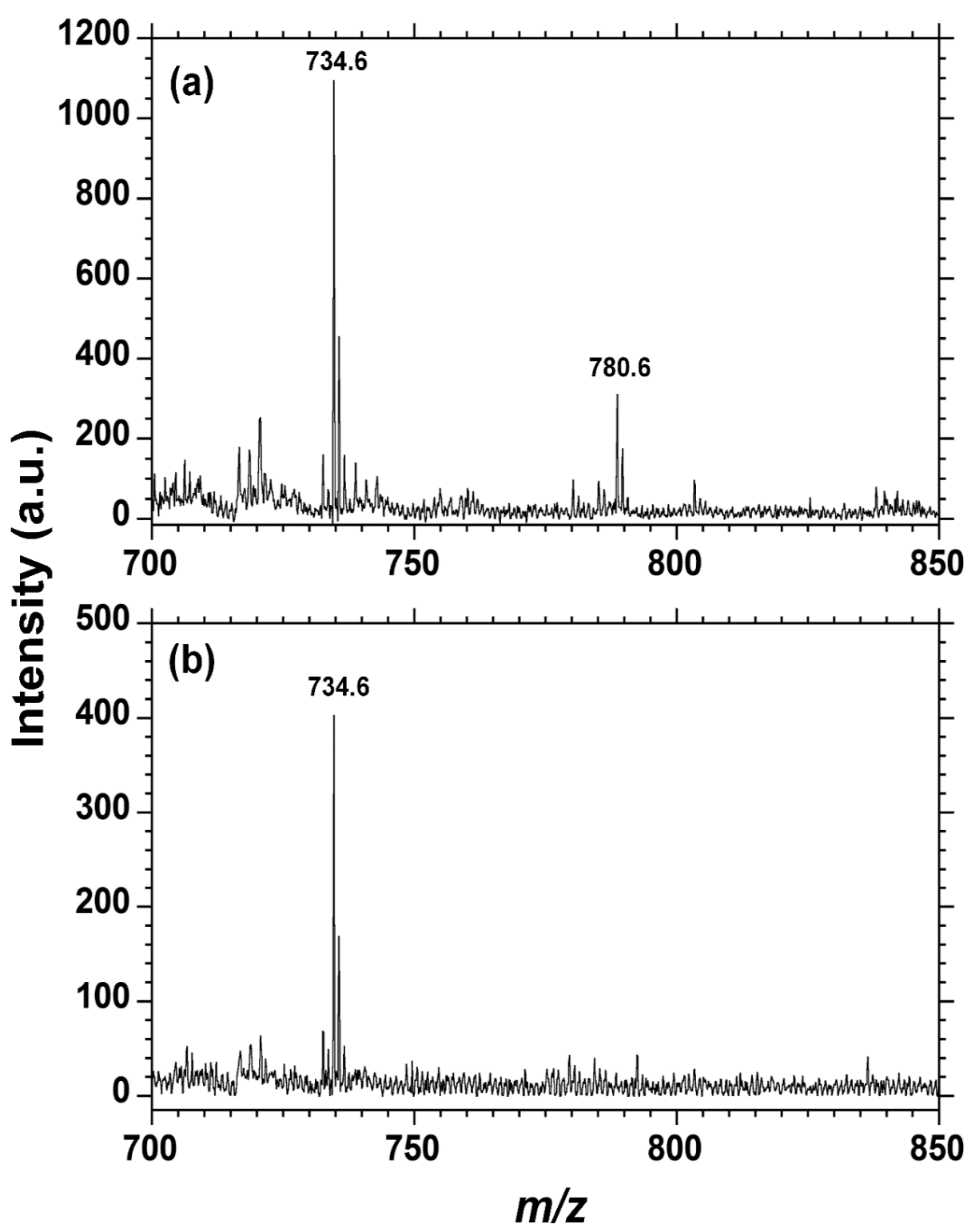

Figure 4-7. Off-line MALDI mass spectra from two different areas (a) capture spot 1 and (b) capture spot 2, on rat brain tissue sample captured using near-field laser ablation sample transfer. 
It has been reported that the average adult rat brain cell is between $10 \mu \mathrm{m}$ and $20 \mu \mathrm{m}$ diameter based on the types and the functions of the cells. ${ }^{252-253}$ Although, we did not target on a specific cells or specific region on mouse brain tissue section, the crater dimensions indicate removal of pico-grams of material from sub-cellular regions. However, in this study, no higher masses were detected in MALDI mass spectra with either CHCA or DHB matrix. This may be due to the limit of detection or fragmentation of the large bio molecules during the ablation process.

\subsection{Summary}

In this work, $532 \mathrm{~nm}$ nanosecond pulsed laser TELA and capture was investigated as a means of transferring biomolecules from surfaces at sub-micrometer dimensions for mass spectrometry analysis. It was found that $532 \mathrm{~nm}$ ablation is significantly more efficient than UV ablation using $355 \mathrm{~nm}$. It was observed that the optimum laser polarization was p-polarization (parallel to the AFM tip). These observations are consistent with near-field ablation at a wavelength near the surface plasmon resonance of the gold AFM tip. The laser wavelength may be resonant with the gold AFM tip, and this may be sufficient for transfer of protein molecules from the surface with no fragmentation. A systematic study of the dependence of laser parameters on tip-enhanced laser ablation will be discussed in Chapter 5.

TELA and capture was used to transfer biomolecules under ambient conditions from rat brain tissue sections within sub cellular level region for MS analysis. The ablation crater sizes sampled with TELA on rat brain tissue, are not limited by the spot size of the $1064 \mathrm{~nm}$ laser, and are sufficient for capture, transfer, and detection of PCs for offline MALDI analysis. Mass spectra obtained from rat brain tissue transferred by TELA were similar to that of analyzed by the standard lipid extraction procedure. Indicative lipids are seen in all of the mass spectra, but 
the relative peak intensities appear to vary from point to point. Future studies will include analyzing individual cells or tissue sections within sub micrometer dimensions for identification of other classes of biomolecules such as peptides and proteins by TELA - MALDI / ESI analysis. 


\section{CHAPTER 5. WAVELENGTH AND POLARIZATION DEPENDANCE OF TIP- ENHANCED LASER ABLATION FOR MASS SPECTROMETRY}

The goal of this study was to elucidate the laser parameters that can affect the efficiency of AFM-tip enhanced laser ablation (TELA) sampling for mass spectrometry. Initial studies of TELA for mass spectrometry by our group as well as others have relied on ultraviolet lasers. From the work described in Chapter 4, we discovered that visible lasers are more efficient for near-field laser ablation sampling. In this chapter, a systematic study of the efficiency of TELA sample transfer at visible and near IR wavelengths and as a function of laser fluence, polarization, and other parameters is described.

\subsection{Introduction}

Analytical techniques related to sub-micrometer scale sampling of material can be useful for acquiring localized chemical information. Far-field laser ablation sampling approaches have been coupled to variety of applications. ${ }^{51,150,254-255}$ However, as conventional far-field laser ablation sampling techniques reach their limits, the need arises for alternative approaches to sample material on the sub-micrometer scale from the surfaces. ${ }^{256}$ As discussed in Chapter 1 , at sub-micrometer scales, far-field optics is limited by diffraction. Furthermore, focusing a beam of light in the far field results in a spot size larger than $\lambda / 2$, where $\lambda$ is the wavelength of incident light. $^{257}$ The localized optical interaction between a probe tip and sample are exploited for material ablation on a sub-micrometer scale below the diffraction limits. ${ }^{67,258}$ There is a need for a systematic study in order to better understand TELA as a sub-micrometer sampling method.

Understanding the fundamental processes of near-field laser ablation can be useful in order to apply near-field laser ablation approaches in many applications, such as micromachining, ${ }^{55,} 259$ pulsed laser deposition, ${ }^{260}$ nanopatterning, ${ }^{261-262}$ as well as chemical 
analysis, where better lateral resolution is needed. For example, near-field laser ablation sampling can be coupled with inductively coupled plasma mass spectrometry (LA-ICP-MS) ${ }^{263}$ and optical emission spectrometry (LA-ICP-OES), ${ }^{264}$ where laser ablation is used as solid sample introduction method. Thus, lateral resolution of the sampling process can be improved by material ablation on a sub-micrometer scale below the diffraction limit. ${ }^{265}$

The utility of aperture mode near-field laser ablation has been demonstrated using scanning near-field optical microscopy (SNOM) probes. ${ }^{210-212,}{ }^{266}$ An alternative approach to aperture mode near-field laser ablation, apertureless near-field laser ablation, has been used to sample material for laser ablation ICP-MS. ${ }^{216-218,221}$ An apertureless near-field laser ablationbased mass spectrometry has also been demonstrated by Goeringer et al. ${ }^{224}$ Although, submicrometer scale resolution has been achieved, the approaches mentioned above were limited to small molecule chemical analysis and often elemental analysis. Therefore, high resolution nearfield laser ablation chemical analysis of biomolecules is challenging and needs much more attention.

In Chapter 3 and Chapter 4, apertureless near-field laser ablation for MALDI mass spectrometry is described. ${ }^{231,244}$ Wavelengths of 355, 532, and $1064 \mathrm{~nm}$ from a Nd:YAG laser were used for near-field ablation. Craters approximately $1 \mu \mathrm{m}$ in diameter were produced. The work presented in Chapters 3 and 4 suggests that visible lasers are more efficient for biomolecule sampling using TELA. Although, AFM-TELA has been utilized for sampling of biomolecules for MS analysis, a systematic study on the efficiency of laser ablation sample transfer as a function of laser fluence, polarization, and other parameters has not been reported. 
Herein, a systematic investigation of AFM-TELA molecular sampling with the aim of maximizing the sampling efficiency is described. Laser wavelength, laser polarization, fluence, and number of laser shots are shown to be important factors in TELA sampling for MS analysis.

\subsection{Experimental}

The AFM laser ablation configuration described in Chapter 2 was used in all experiments reported in this chapter. The distance between AFM tip and sample surface was controlled by engaging the AFM probe in tapping mode. The ablation laser is a pulsed nanosecond Nd:YAG laser, at $532 \mathrm{~nm}$ or $1064 \mathrm{~nm}$ operated in a single shot mode. The laser polarization was adjusted using a half wave plate. The laser was focused approximately $4 \mathrm{~cm}$ beyond the AFM probe and the resulting laser diameter where it intersected the tip was $7 \mathrm{~mm}$. The laser energy was attenuated externally using a variable neutral density filter.

For all experiments reported in this chapter, anthracene was used as the test material. The anthracene crystals formed on a microscope cover slip were used for the analysis. The AFM images of the area of interest were obtained prior to the TELA and after the ablation experiments for comparison.

\subsection{Results}

The initial goal was to understand the physical phenomena behind AFM-TELA using different laser wavelengths and optimize the ablation efficiency. The work reported in Chapters 3 and 4 demonstrates that sub-micrometer level sampling of biomolecules is possible with AFMTELA using a Nd:YAG laser at $355 \mathrm{~nm}$ and $532 \mathrm{~nm}$. In this work, the efficiency of the TELA ablation at $532 \mathrm{~nm}$ and $1064 \mathrm{~nm}$ wavelengths was studied as a function of laser fluence, polarization, and other parameters. 
Initial work concentrated on optimizing the efficiency of TELA by systematically changing laser polarization, fluence, and number of laser shots. Two extremes in orientation are possible for the laser polarization, either parallel laser polarization with respect the tip axis (ppolarization) or horizontal laser polarization with respect to the tip axis (s-polarization). First, the laser polarization was set to p-polarization and the ablation was performed with laser fluence of $3.5 \mathrm{~kJ} / \mathrm{m}^{2}$. The polarization of the light was then rotated to s-orientation and ablation was performed with the same laser fluence. AFM height images of the ablation craters produced using $\mathrm{p}$ and s laser polarizations are shown in Figure 5-1. Profile analysis of the resulting AFM height images indicated that the p-polarized laser resulted a larger crater (Figure 5-1 a) as compared to the ablation crater produced with s-polarized light (Figure 5-1 b). Polarization rotated by $180^{\circ}$ produced identical crater as compared to $0^{\circ}$ rotation (Figure $5-1 \mathrm{c}$ ), indicating absence of any anisotropy involved in optics.
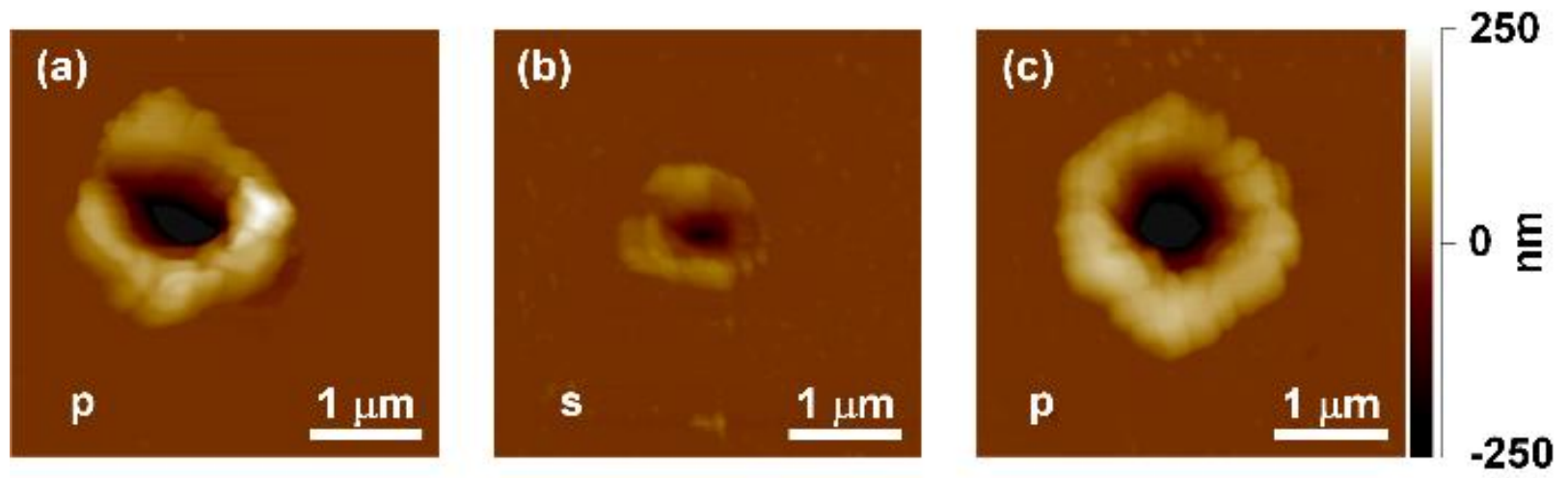

Figure 5-1. AFM images of ablation crater at different polarization angles 
Next, the ablated volume was measured as a function of laser polarization. The laser fluence was $3.5 \mathrm{~kJ} / \mathrm{m}^{2}$ and laser polarization angle was rotated from p-polarization to spolarization and the crater volumes were measured at each angle of rotation. Ablation crater volumes were calculated from the average crater areas and depths using AFM height images and assuming conical craters. Figure 5-2 shows the plot of crater volume as a function of polarization angle, where $0^{\circ}$ and $180^{\circ}$ rotation represent p-polarization. Crater volumes gradually decreased as the stepwise rotation of the polarization angle from p-polarization to s-polarization and

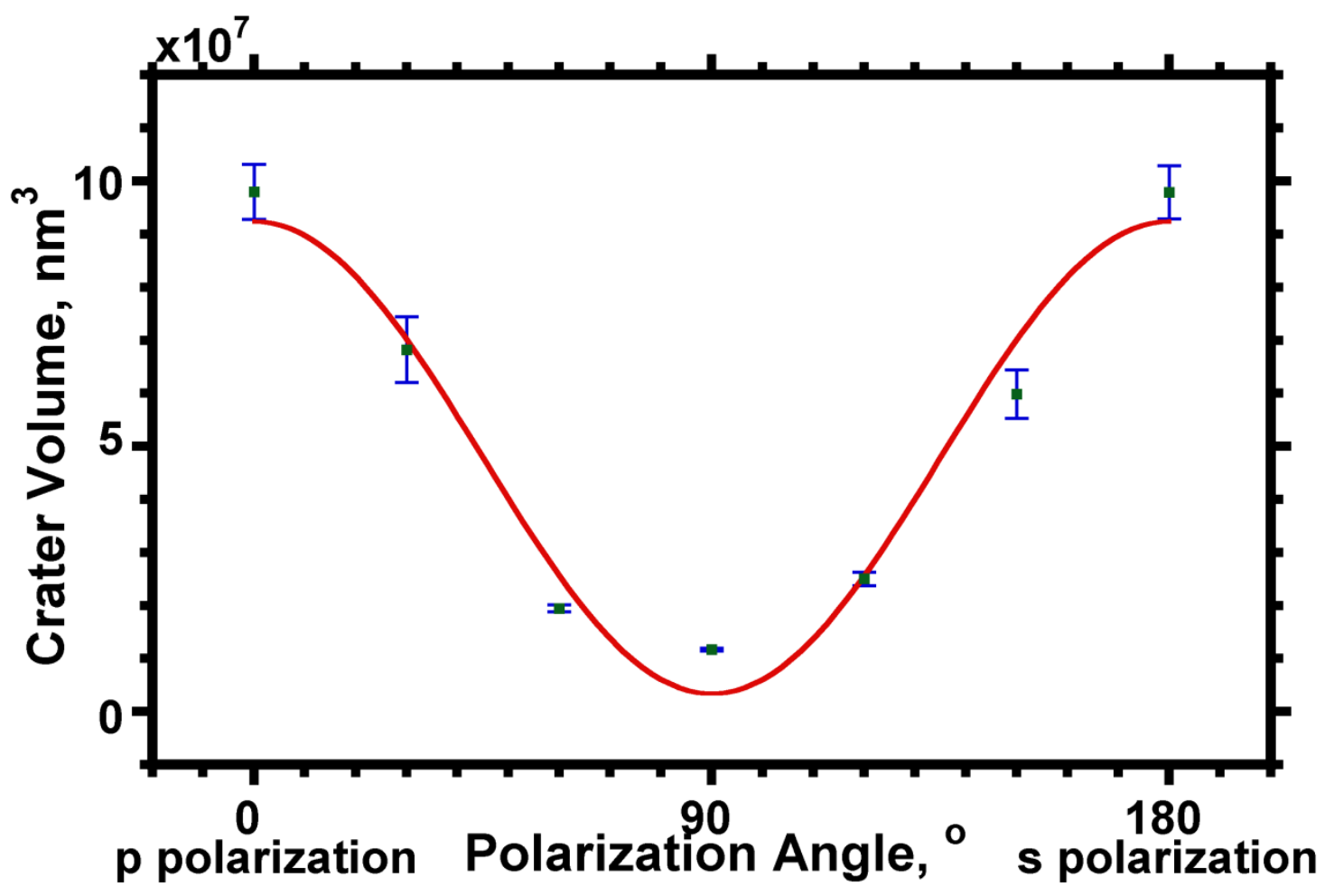

Figure 5-2. Ablation crater volume plotted as a function of rotation of polarization of the laser. $0^{\circ}$ and $180^{\circ}$ represents p-polarization, whereas $90^{\circ}$ is s-polarization in the plot. 
reached to minima when the laser polarization is perpendicular to the tip axis (s polarization). It has been reported that polarization dependent ablation signals of a near-field tip have a $\cos ^{2} \theta$ dependence. ${ }^{267-268}$ The data in Figure 5-2 were fit with $\cos ^{2} \theta$ dependence. Several near-field experiments as well as simulation studies have shown that surface plasmon generation at the probe apex is maximized when the laser polarization is parallel to the probe axis. ${ }^{269-272}$

The initial experiments on TELA were performed with $355 \mathrm{~nm}$ wavelength and no ablation was observed with p-polarized light. A comparison of anthracene ablation craters formed by $532 \mathrm{~nm}$ and $355 \mathrm{~nm}$ wavelengths are shown in Figure 5-3. For this comparison, the $532 \mathrm{~nm}$ laser was used with p-polarized light and the sample was ablated using a single laser shot at a fluence of $850 \mathrm{~J} / \mathrm{m}^{2}$. For the $355 \mathrm{~nm}$ laser, s-polarized light with a laser fluence of $850 \mathrm{~J} / \mathrm{m}^{2}$ was used and 30 laser shots were required to obtain a comparable crater. Furthermore, rapid tip damage is also observed with $355 \mathrm{~nm}$ wavelength while the tip damage was considerably less with 532 nm laser (see Figure 2-3).
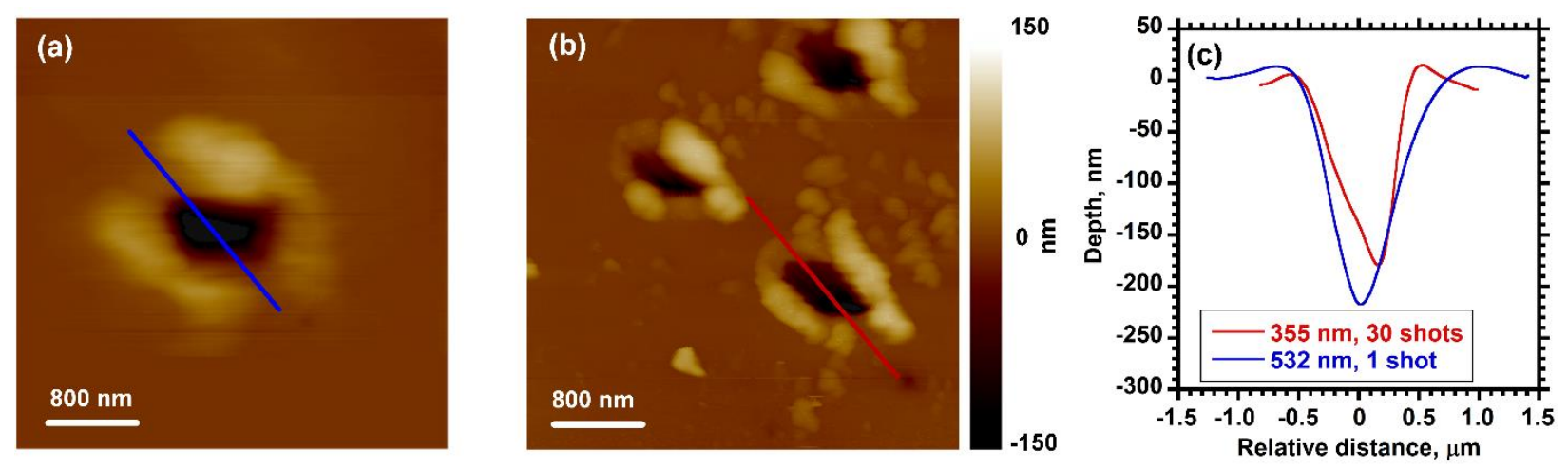

Figure 5-3. AFM-TELA at different wavelengths (a) ablation crater with a single shot of $532 \mathrm{~nm}$ laser wavelength at $850 \mathrm{~J} / \mathrm{m}^{2}$ fluence, (b) example of ablation craters with 30 shots of $355 \mathrm{~nm}$ laser at $850 \mathrm{~J} / \mathrm{m}^{2}$ fluence. (c) Comparison of depth profile of the craters created with $355 \mathrm{~nm}$ and $532 \mathrm{~nm}$ laser wavelength. 
The studies performed with the Nd:YAG laser at $1064 \mathrm{~nm}$ resulted in ablation craters with no significant difference regardless of the polarization. However, single laser shot ablation at $1064 \mathrm{~nm}$ required $10 \mathrm{~kJ} / \mathrm{m}^{2}$ laser fluence, compared to $850 \mathrm{~J} / \mathrm{m}^{2}$ fluence at $532 \mathrm{~nm}$ wavelength, to produce a comparable ablation crater. We attribute this to the combination of the excitation of the plasmon resonance polaritons using parallel polarization and stronger plasmon absorption of the gold-coated AFM tip at $532 \mathrm{~nm}$ as compared to other wavelengths tested. ${ }^{273-274}$

The laser fluence dependence was investigated by measuring the ablation crater volume as a function of laser fluence. The anthracene samples were ablated using a single laser shot at $532 \mathrm{~nm}$ laser with p-polarization. Figure 5-4 shows a plot of the volume of the ablation crater as a function of laser fluence. A weak ablation regime was observed between 0.6 and $3 \mathrm{~kJ} / \mathrm{m}^{2}$, and a

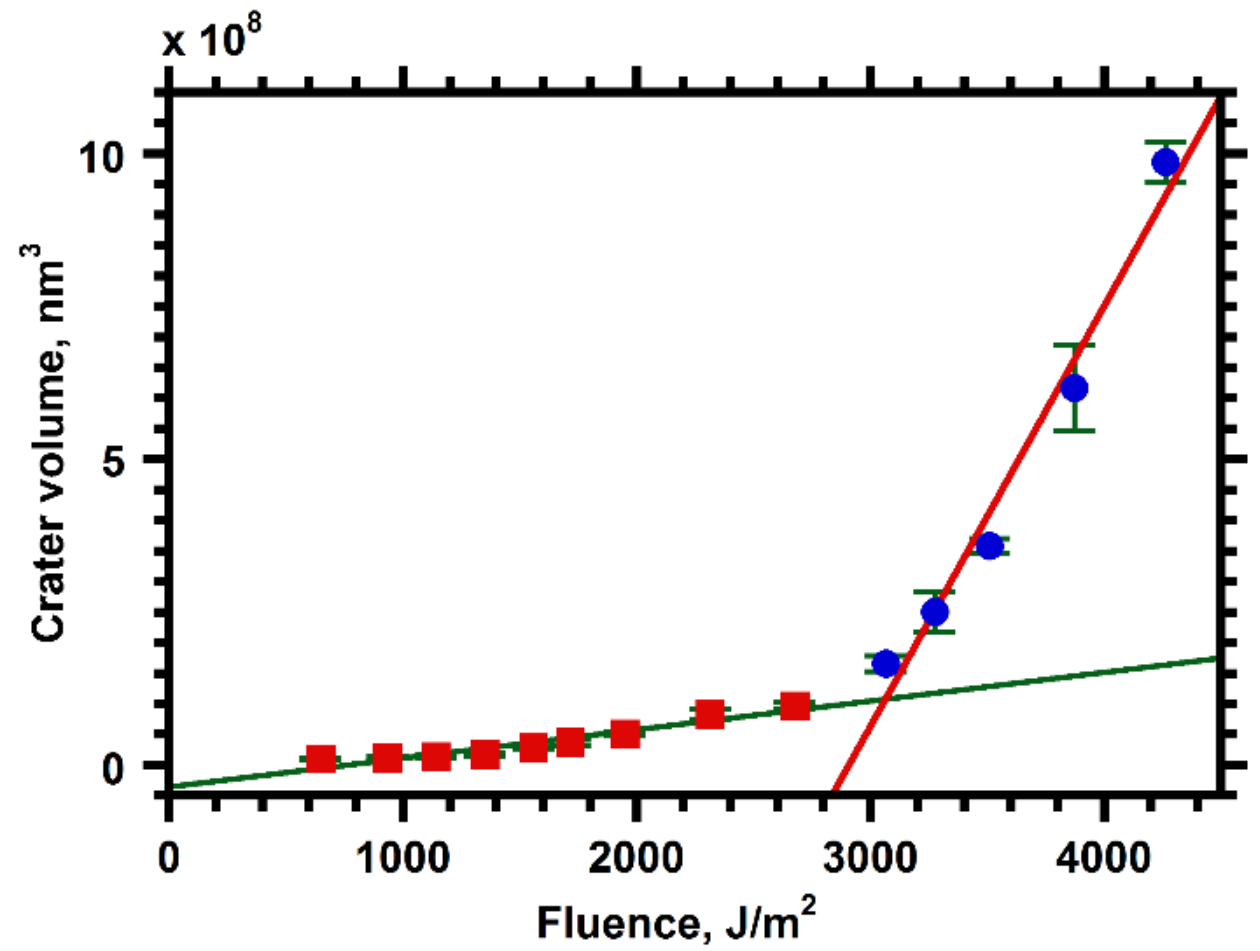

Figure 5-4. Volume of the ablation crater as a function of laser fluence at $532 \mathrm{~nm}$ 
significant increase in the slope was observed approximately at $3 \mathrm{~kJ} / \mathrm{m}^{2}$. Further increase in the laser fluence above $4.5 \mathrm{~kJ} / \mathrm{m}^{2}$ resulted tip damage.

For comparison, the laser fluence dependence on TELA was tested using $1064 \mathrm{~nm}$ wavelength. The anthracene samples were ablated using a single laser shot at $1064 \mathrm{~nm}$ with $\mathrm{p}$ polarization. Figure 5-5 shows the plot of the volume of the ablation crater as a function of laser fluence. A similar trend was observed as compared to $532 \mathrm{~nm}$ wavelength. It is important to note that $\sim 10$ fold higher laser fluence compared to $532 \mathrm{~nm}$ was needed to ablate material using 1064 $\mathrm{nm}$.

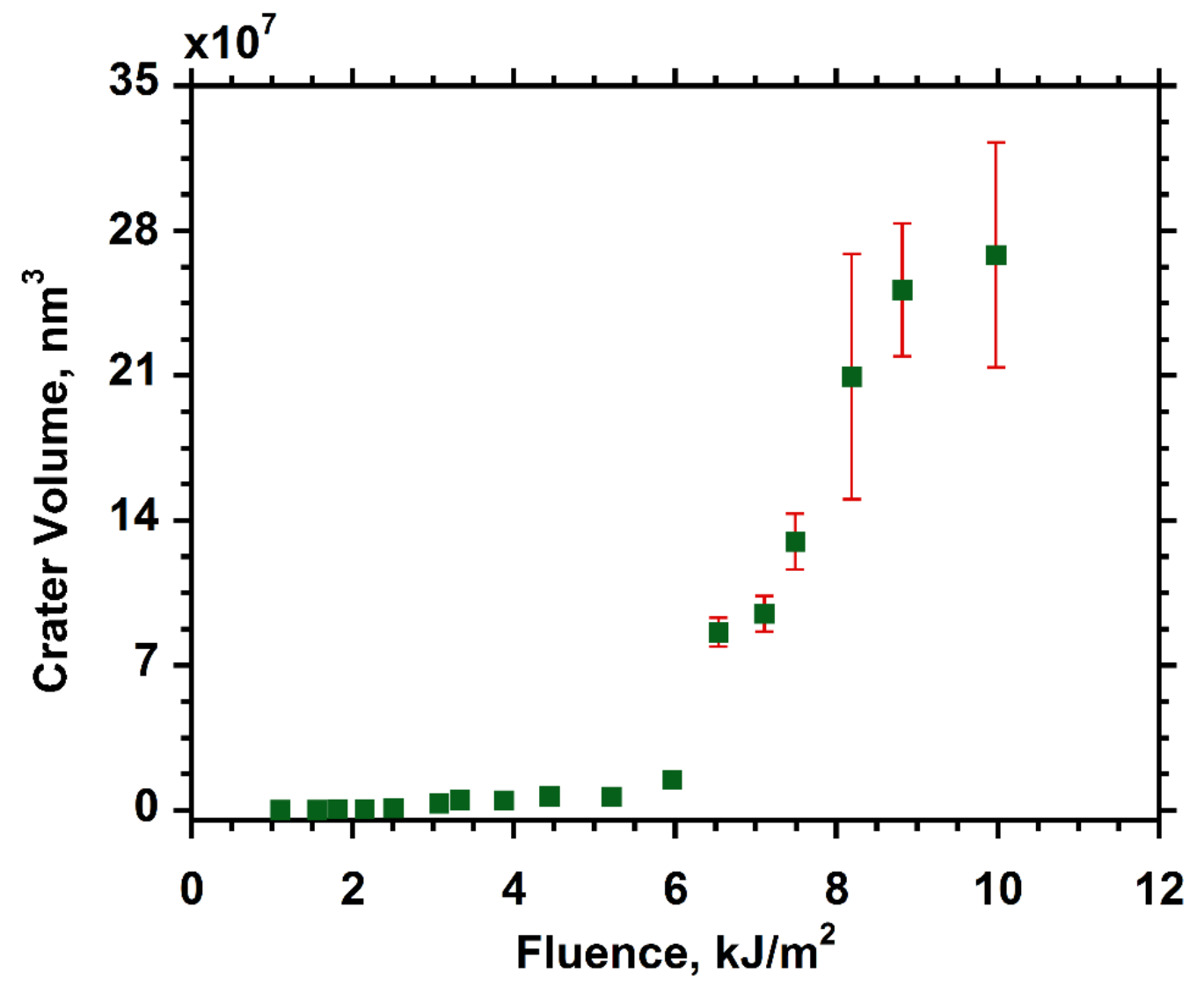

Figure 5-5. Volume of the ablation crater is plotted as a function of laser fluence using $1064 \mathrm{~nm}$ wavelength laser. A clear change of slope is observed at particular threshold fluence. 
Several studies on near-field enhancement under femtosecond laser irradiation have reported the linear relationship between laser fluence and the field enhancement. ${ }^{270,}$ 275-276 Harrison and Yakar demonstrated ablation of silicon using gold nano rods. ${ }^{275}$ The material ablation was significantly increased after the threshold fluence and it was linearly increased and reached to a maxima.

Robitaille et al suggest that the mechanism of plasmon enhanced laser ablation is related to the generation of highly localized energetic carriers. ${ }^{276}$ At higher laser fluence, ablation is dominated by the energy transfer from the localized electric field at the near-field element to the sample through the diffusion of highly localized energetic carriers and generating ablation. Therefore, we believe that the significant ablation above $3 \mathrm{~kJ} / \mathrm{m}^{2}$ laser fluence is caused by the excitation of highly localized plasmon resonance polaritons at the tip. In the case of $1064 \mathrm{~nm}$, higher energy was required to excite plasmons since weaker plasmon absorption of the goldcoated AFM tip at $1064 \mathrm{~nm}$.

Next, the number of laser shots needed for the maximum amount of material removal was investigated at a fixed laser fluence. Figure 5-6, shows the plot of ablation crater volumes as a function of number of shots at three different laser fluences using p-polarized $532 \mathrm{~nm}$ light. For higher laser fluences, a rapid increase in the crater volume was observed while lower fluences showed slow increase in crater volume with increasing number of shots. Interestingly, the change in the crater volume reached to maxima for all three laser fluences after 10 consecutive laser shots. This is more likely to be due to the tip being shadowed inside the ablation crater. We recall that the tip-surface distance was controlled by engaging the AFM probe in tapping mode. Hence, the AFM probe was oscillating between 0-15 $\mathrm{nm}$ from the surface at its resonance frequency during the laser irradiation. Therefore, the AFM tip is fully exposed to the incident 
laser during the first few laser shots. Hence, a rapid ablation is expected during the first few laser shots. Therefore, no further ablation occurs after 10 laser shots. Trials above $3 \mathrm{~kJ} / \mathrm{m}^{2}$ threshold fluence resulted tip damage after 5 consecutive laser shots. These observations indicated not only the importance of number of laser shots for maximizing the ablation crater size, but also the importance of laser fluence for maximizing crater dimensions. Herein, we demonstrate the ability of controlling the ablation crater size in the range of $500 \mathrm{~nm}$ to $3 \mu \mathrm{m}$ by tuning laser parameters and number of laser shots.

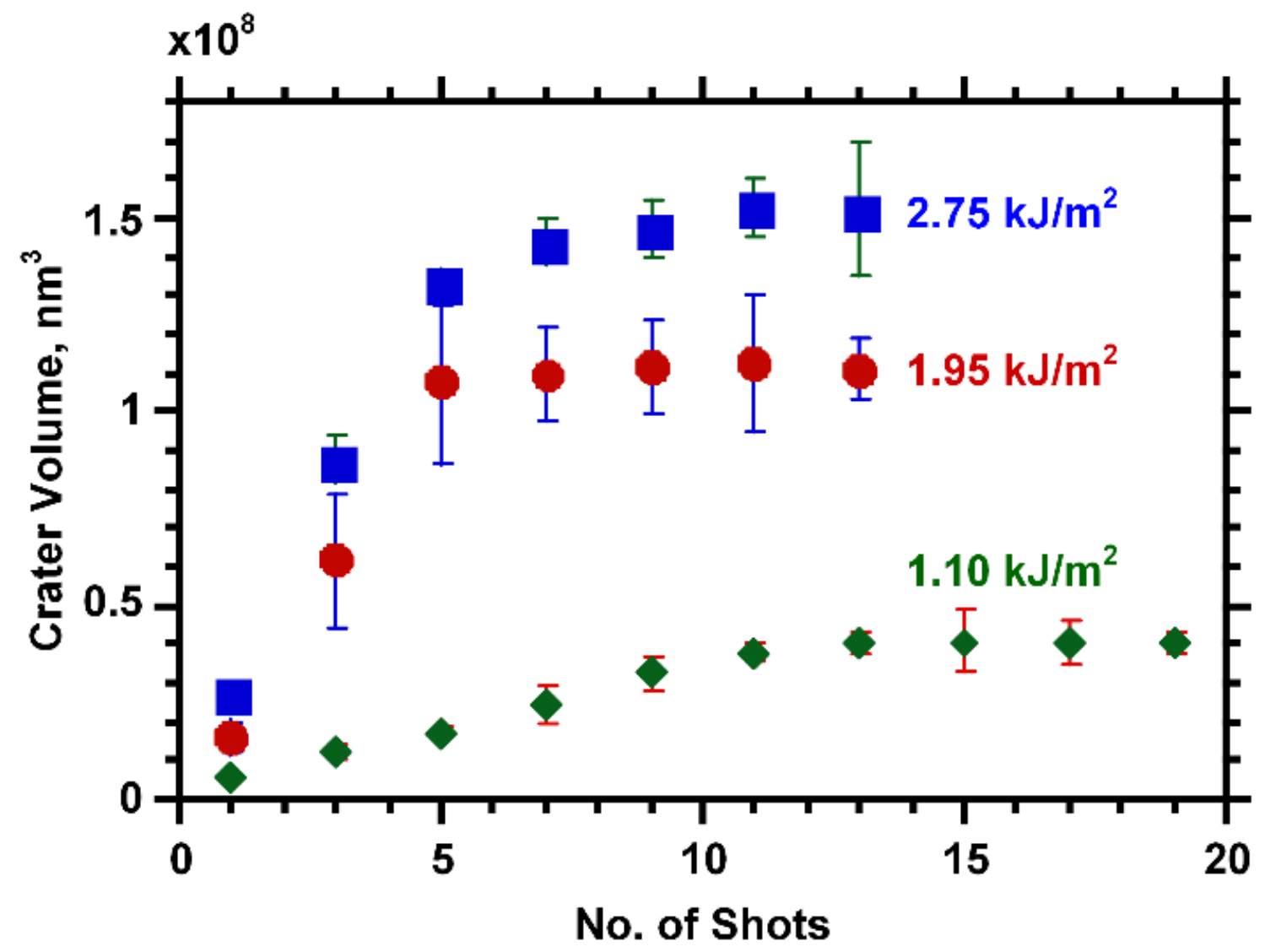

Figure 5-6. Volume of material ablated is plotted as a function of number of laser shots at different laser fluences. 
Since laser far-field ablation was not observed for laser pulses when the AFM tip is not in the vicinity of the sample surface. It suggests that the ablation process is enhanced by the AFM tip. There is a tip enhancement factor for ablation under the same laser irradiation when the tip is engaged within $15 \mathrm{~nm}$ above the surface. Therefore, we define a tip enhancement factor (TEF) by finding the ratio between total energy required to make a single ablation crater to total available energy during the ablation process. An estimation of the tip enhancement factor (TEF) during the laser irradiation is more advantageous in order to understand the efficiency of TELA at optimized conditions. Herein, we report an estimation of $T E F$ using the thermodynamic values and absorption coefficient of anthracene at $532 \mathrm{~nm}$ wavelength. The total energy $\left(\Delta_{\boldsymbol{t o t}} \boldsymbol{H}\right)$ requirement for the phase change of anthracene (from crystalline solid state to gas phase) can be calculated using Equation 5.1.

$$
\Delta_{t o t} H=\Delta E_{l a t t}+m s \Delta T_{m}+\Delta_{f u s} H+m s \Delta T_{b}+\Delta_{v a p} H
$$

Where, $\Delta E_{\text {latt }}$ is the lattice energy, $\Delta_{f u s} H$ is the heat of fusion, and $\Delta v a p H$ is the heat of vaporization of anthracene. The mass and specific heat of anthracene is given by $m$ and $s$ respectively. $\Delta T_{m}$ and $\Delta T_{b}$ are the temperature change for melting and vaporization. We can also estimate the energy available at the sample surface without the tip enhancement using the laser spot diameter, threshold laser fluence, and absorption cross-section ${ }^{277}$ of anthracene. Therefore, the $T E F$ can be calculated from the ratio of $\Delta_{t o t} H$ and the available energy without tip enhancement, was found to be $10^{4}$ fold. The estimated $T E F$ is comparable with the probe enhancement reported by Furukawa and Kawata, ${ }^{272}$ However, several other experimental and simulation studies have revealed that enhancement factor estimation is very much subject to the 
modeling of the tip for simulations and experimental parameters with high order of dependency. $^{278}$

AFM height images of the ablation craters indicated displacement of the ablated material around the rim of the ablation crater. However, successive imaging around the ablation crater in tapping mode indicated that the crater rim material is low density material generated during ablation. Figure 5-7 shows the comparison of the AFM height image in tapping mode after the first scan (Figure 5-7 a) and fifth scan (Figure 5-7 b). The amount of displaced ablated material has decreased as the number of AFM scans increased, suggesting that the crater material is low density material formed during the ablation process.
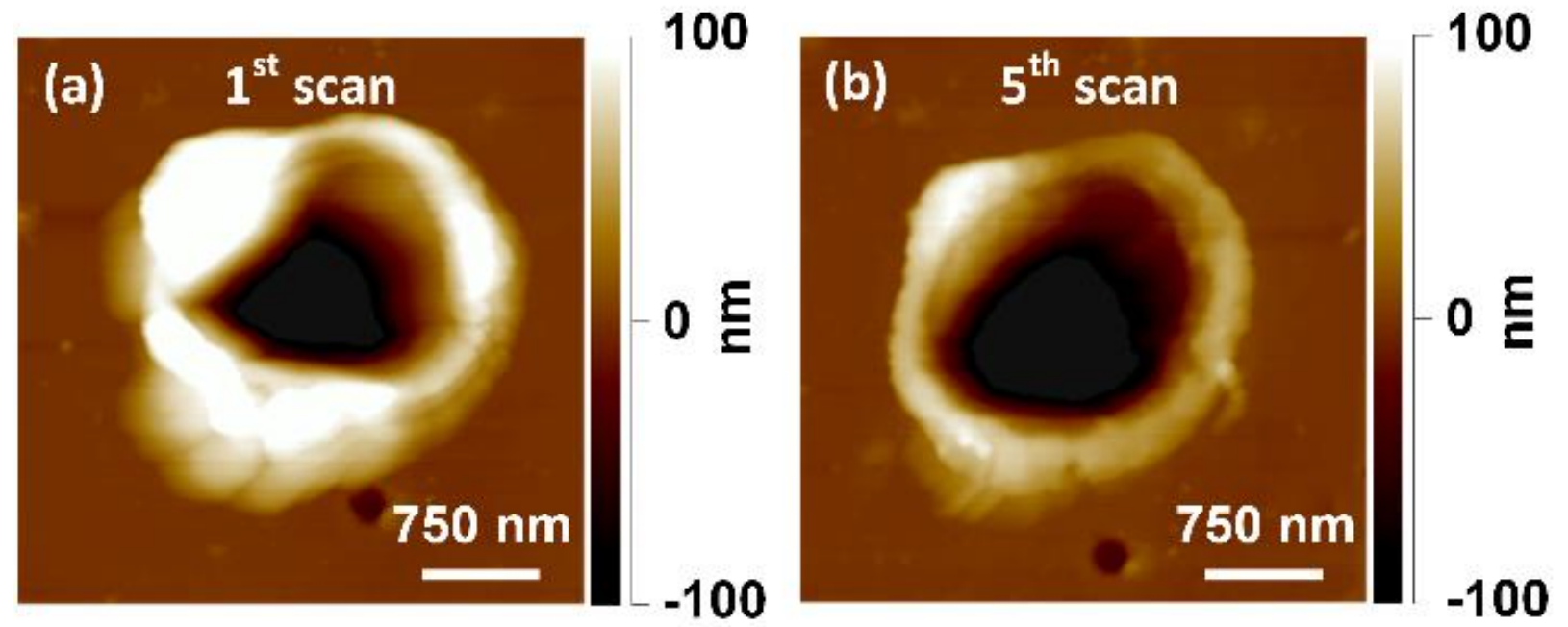

Figure 5-7. Material deposited around the ablation craters (a) First AFM scan after ablation showing melting on the perimeter of the crater. (b) Same crater has been scanned for 5 times and the amount of amount of melted materials decreased considerably, indicating very low density of the melted material. 


\subsection{Summary}

The utility of AFM-TELA as a localized sampling system for molecular analysis marks a substantial improvement in sub-micrometer scale sampling of molecules. We have demonstrated the capabilities of TELA method to tailor the ablation crater dimensions for sub-micrometer sized sampling by tuning the laser parameters. Systematic studies of ablation efficiency by changing laser polarization yield important insights for the ablation process: first, the field enhancement of the probe is polarization dependent, and, second, the polarization dependence relies on the laser wavelength. By changing the wavelength, we have demonstrated that $532 \mathrm{~nm}$ is more efficient compared to $355 \mathrm{~nm}$ for AFM-TELA with gold coated probes. The TEF was calculated, using experimentally determined ablation crater volume and the thermodynamic properties of anthracene, to be $10^{4}$ fold. With the combination of proper polarization, wavelength, and laser fluence, it is possible to ablate material with sub-micrometer dimensions, for sampling of molecules for chemical and biomedical analysis. 


\section{CHAPTER 6. CONCLUSIONS AND FUTURE DIRECTIONS}

In this dissertation, the development of atomic force microscope tip-enhanced laser ablation (AFM TELA) for sub micrometer level ambient sampling of material for MS was described. The significance of this work lies in the ability to ablate large biomolecules on a submicrometer scale without fragmentation for off-line mass spectrometry.

In Chapter 3, TELA was used with a $355 \mathrm{~nm}$ pulsed Nd:YAG laser to ablate small molecules, allowing a good initial performance assessment. Anthracene and rhodamine $6 \mathrm{G}$ dye, commonly used ablation standards, were used. Removal of material from sub-micrometer size ablation craters, topographical images of the ablation craters, and direct laser desorption ionization (LDI) were demonstrated. LDI of anthracene and rhodamine 6G showed that the molecules were captured intact. The capture efficiency was estimated to be approximately $3 \%$.

The detection of intact biomolecules and lipids from rat brain tissue using TELA was described in Chapter 4. To detect captured biomolecules by TELA MS, matrix addition and deposition were necessary. TELA-MS studies demonstrated that biomolecules up to ca. $6000 \mathrm{Da}$ can be analyzed. In contrast to laser ablation transfer at $355 \mathrm{~nm}$, fragments were not observed for insulin ablation transfer at $532 \mathrm{~nm}$ laser wavelength. The gentler material transfer at $532 \mathrm{~nm}$ as compared to $355 \mathrm{~nm}$ is attributed to the stronger plasmon absorption at this wavelength for the $30 \mathrm{~nm}$ gold coated AFM tip.

The utility of TELA for off-line mass spectrometry was tested with sections of rat brain tissue at $1064 \mathrm{~nm}$. The TELA-MS data of the rat brain tissue analysis show distinctive lipid peaks attributed to sub-micrometer ablation of biomolecules on rat brain tissue.

A systematic study of the laser wavelength and polarization dependence of TELA was presented in Chapter 5. It was found that $532 \mathrm{~nm}$ is more efficient compared to $355 \mathrm{~nm}$ tip- 
enhanced laser ablation, possibly due to the better overlap with the gold surface plasmon absorption. One of the most significant differences between $532 \mathrm{~nm}$ and $355 \mathrm{~nm}$ ablation is the strong laser polarization dependence at $532 \mathrm{~nm}$, indicative of a near-field effect. With $532 \mathrm{~nm}$ radiation, tip parallel (p-polarization) is favored and removes more than five times as much material as tip perpendicular (s-polarization), consistent with a near-field effect. Infrared ablation using $1064 \mathrm{~nm}$ radiation was used to ablate material in tip-enhanced mode, but required approximately 10 times more pulse energy compared to $532 \mathrm{~nm}$ and $355 \mathrm{~nm}$.

One of the future directions will focus on merging the ablated material with electrospray. The plume of ablated material from the AFM TELA system can be coupled with an electrospray for on-line ionization. A sampling capillary can transport the ablated material into the interface to interact with electrospray, which is then ionized through an electrospray evaporation process. ${ }^{148,}$ 279-280 Although, merged electrospray ionization on-line MS interfaces are commercially available, higher flow rates associated with these systems are problematic for detecting atto-grams of material captured by TELA. Sub micrometer ablation of large biomolecules requires extremely low flow rates in order to maintain the limit of detection (LOD) and sensitivity of detection. Commercially available nano-electrospray tips can be used (typically sprays $1 \mathrm{~nL} / \mathrm{min}$ ) as the low flow electrospray emitter while maintaining the sensitivity and LOD for on-line TELA-MS. One of the key advantages of adopting off-line sub-micrometer ablation of large biomolecules for MS to an on-line electrospray source include, ablated large biomolecules can be ionized and detected without matrix, which will reduce background signal. Sub-micrometer ablation of large biomolecules using TELA does not require an addition of a matrix for efficient ablation. 
A second future direction will focus on precise sampling material using TELA for genomics. TELA can be useful for extremely precise sampling of biomolecules and is not limited to MS detection. Therefore, additional studies using TELA can be conducted with genetic material such as DNA to capture, transfer, amplify using PCR, and detect using gel electrophoresis. Figure 6-1 represents the preliminary data using TELA for genomic studies.

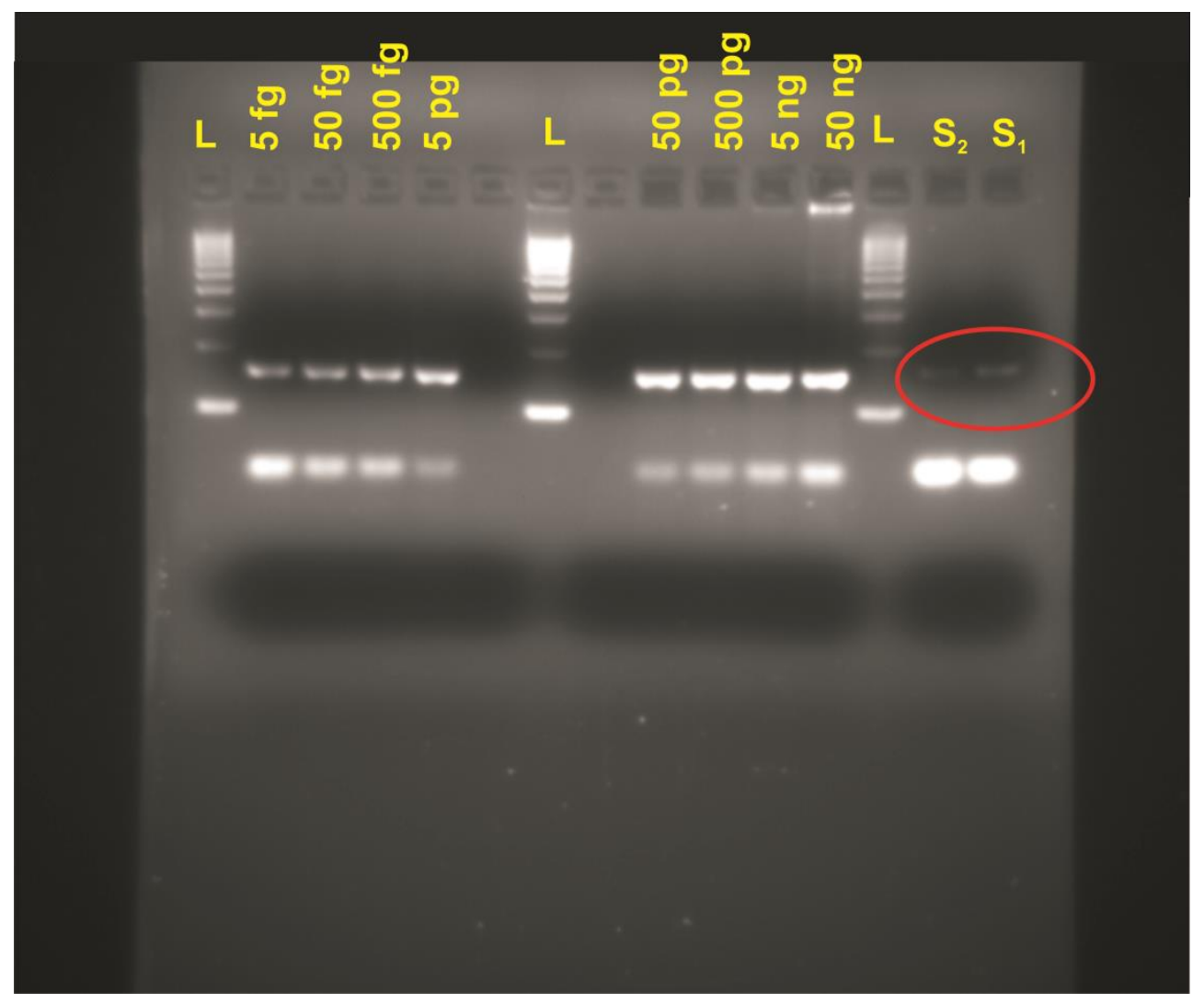

Figure 6-1. Gel electrophoresis of PCR products of TELA captured DNA plasmids. The ladders are 100 base pairs spaced, with the lowest value of 100 and the ladder wells are denoted with letter L. S1 and S2 are the PCR products of the TELA captured samples respectively. The PCR product that we monitor is circled. Different amounts of the PCR product of standard pGEM are used for quantification. 
DNA plasmids were ablated using TELA, captured, amplified and detected using gel electrophoresis. The initial ablation capture process shows that genetic material can be captured for genomics using TELA. If sufficient amount of genetic material are transferred, nucleic acid sequence of the captured genetic material can also be obtained through next generation sequencing. ${ }^{281}$ Furthermore, the TELA approach will be extremely useful for precise sampling of genetic materials from single cells and tissues. Current techniques such as micro-manipulation, fluorescence based cell sorting and laser capture micro-dissection involve critical cell separation steps for single cell DNA sequencing; whereas TELA can be used to sample genetic material in situ directly from tissue samples. Hence, this technique can significantly simplify single cell DNA sequencing and RNA quantification. 


\section{REFERENCES}

1. de Hoffmann, E.; Stroobant, V., Mass Spectrometry: Principles and Applications; Wiley, 2007.

2. El-Aneed, A.; Cohen, A.; Banoub, J., Mass Spectrometry, Review of the Basics: Electrospray, MALDI, and Commonly Used Mass Analyzers. Appl. Spectrosc. Rev. 2009, 44, 210-230.

3. Watson, J. T.; Sparkman, O. D., Introduction to Mass Spectrometry: Instrumentation, Applications, and Strategies for Data Interpretation; John Wiley \& Sons, 2007.

4. Vestal, M. L., Methods of Ion Generation. Chem. Rev. 2001, 101, 361-376.

5. Banerjee, S.; Mazumdar, S., Electrospray Ionization Mass Spectrometry: A Technique to Access the Information Beyond the Molecular Weight of the Analyte. Int. J. Anal. Chem. 2012, 2012.

6. Hillenkamp, F.; Peter-Katalinic, J., MALDI MS: A Practical Guide to Instrumentation, Methods and Applications; John Wiley \& Sons, 2013.

7. Tanaka, K., The Origin of Macromolecule Ionization by Laser IR radiation (Nobel Lecture). Angew. Chem. Int. Ed. 2003, 42, 3860-3870.

8. Geer, L. Y.; Markey, S. P.; Kowalak, J. A.; Wagner, L.; Xu, M.; Maynard, D. M.; Yang, X.; Shi, W.; Bryant, S. H., Open Mass Spectrometry Search Algorithm. J. Proteome Res. 2004, 3, 958-964.

9. Mann, M.; Hendrickson, R. C.; Pandey, A., Analysis of Proteins and Proteomes by Mass Spectrometry. Annu. Rev. Biochem. 2001, 70, 437-473.

10. Mishur, R. J.; Rea, S. L., Applications of Mass Spectrometry to Metabolomics and Metabonomics: Detection of Biomarkers of Aging and of Age - Related Diseases. Mass Spectrom. Rev. 2012, 31, 70-95.

11. Hyung, S. J.; Ruotolo, B. T., Integrating Mass Spectrometry of Intact Protein Complexes into Structural Proteomics. Proteomics 2012, 12, 1547-1564.

12. Chaurand, P., Imaging Mass Spectrometry of Thin Tissue Sections: A Decade of Collective Efforts. J. Proteomics 2012, 75, 4883-4892. 
13. Caprioli, R. M.; Farmer, T. B.; Gile, J., Molecular Imaging of Biological Samples: Localization of Peptides and Proteins Using MALDI-TOF MS. Anal. Chem. 1997, 69, 4751-4760.

14. Caprioli, R. M., Imaging Mass Spectrometry: Enabling a New Age of Discovery in Biology and Medicine through Molecular Microscopy. J. Am. Soc. Mass Spectrom. 2015, $26,850-852$.

15. McDonnell, L. A.; Heeren, R., Imaging Mass Spectrometry. Mass Spectrom. Rev. 2007, 26, 606-643.

16. Watrous, J. D.; Dorrestein, P. C., Imaging Mass Spectrometry in Microbiology. Nature Rev. Microbiol. 2011, 9, 683-694.

17. Solon, E. G.; Schweitzer, A.; Stoeckli, M.; Prideaux, B., Autoradiography, MALDI-MS, and SIMS-MS Imaging in Pharmaceutical Discovery and Development. Am. Assoc. Pharm. Sci. 2010, 12, 11-26.

18. Kaspar, S.; Peukert, M.; Svatos, A.; Matros, A.; Mock, H. P., MALDI - Imaging Mass Spectrometry-an Emerging Technique in Plant Biology. Proteomics 2011, 11, 18401850.

19. Liebl, H., Ion Microprobe Mass Analyzer. J. Appl. Phys. 1967, 38, 5277-5283.

20. Lanni, E. J.; Rubakhin, S. S.; Sweedler, J. V., Mass Spectrometry Imaging and Profiling of Single Cells. J. Proteomics 2012, 75, 5036-5051.

21. Luxembourg, S. L.; Mize, T. H.; McDonnell, L. A.; Heeren, R. M., High-Spatial Resolution Mass Spectrometric Imaging of Peptide and Protein Distributions on a Surface. Anal. Chem. 2004, 76, 5339-5344.

22. Soltwisch, J.; Göritz, G.; Jungmann, J. H.; Kiss, A. s.; Smith, D. F.; Ellis, S. R.; Heeren, R. M., MALDI Mass Spectrometry Imaging in Microscope Mode with Infrared Lasers: Bypassing the Diffraction Limits. Anal. Chem. 2013, 86, 321-325.

23. Svatoš, A., Mass Spectrometric Imaging of Small Molecules. Trends Biotechnol. 2010, $28,425-434$.

24. Watrous, J. D.; Alexandrov, T.; Dorrestein, P. C., The Evolving Field of Imaging Mass Spectrometry and Its Impact on Future Biological Research. J. Mass Spectrom. 2011, 46, 209-222. 
25. Bantscheff, M.; Lemeer, S.; Savitski, M. M.; Kuster, B., Quantitative Mass Spectrometry in Proteomics: Critical Review Update from 2007 to the Present. Anal. Bioanal. Chem. 2012, 404, 939-965.

26. Becker, J. S.; Salber, D., New Mass Spectrometric Tools in Brain Research. TrAC, Trends Anal. Chem. 2010, 29, 966-979.

27. Trouillon, R. 1.; Passarelli, M. K.; Wang, J.; Kurczy, M. E.; Ewing, A. G., Chemical Analysis of Single Cells. Anal. Chem. 2012, 85, 522-542.

28. Winograd, N., The Magic of Cluster SIMS. Anal. Chem. 2005, 77, 142 A-149 A.

29. Brunelle, A.; Touboul, D.; Laprévote, O., Biological Tissue Imaging with Time - of Flight Secondary Ion Mass Spectrometry and Cluster Ion Sources. J. Mass Spectrom. 2005, 40, 985-999.

30. Boxer, S. G.; Kraft, M. L.; Weber, P. K., Advances in Imaging Secondary Ion Mass Spectrometry for Biological Samples. Annu. Rev. Biophys. 2009, 38, 53-74.

31. Fletcher, J. S.; Vickerman, J. C., Secondary Ion Mass Spectrometry: Characterizing Complex Samples in Two and Three Dimensions. Anal. Chem. 2012, 85, 610-639.

32. Cornett, D. S.; Reyzer, M. L.; Chaurand, P.; Caprioli, R. M., MALDI Imaging Mass Spectrometry: Molecular Snapshots of Biochemical Systems. Nat. Methods 2007, 4, 828833.

33. Dreisewerd, K., Recent Methodological Advances in MALDI Mass Spectrometry. Anal. Bioanal. Chem. 2014, 406, 2261-2278.

34. Benninghoven, A., Chemical-Analysis of Inorganic and Organic-Surfaces and ThinFilms by Static Time-of-Flight Secondary-Ion Mass-Spectrometry (TOF-SIMS). Angew. Chem. Int. Ed. 1994, 33, 1023-1043.

35. Winograd, N., The Magic of Cluster SIMS. Anal. Chem. 2005, 77, 142 A-149 A.

36. Bich, C.; Touboul, D.; Brunelle, A., Cluster TOF-SIMS Imaging as a Tool for Micrometric Histology of Lipids in Tissue. Mass Spectrom. Rev. 2013.

37. Fletcher, J. S.; Vickerman, J. C., Secondary Ion Mass Spectrometry: Characterizing Complex Samples in Two and Three Dimensions. Anal. Chem. 2013, 85, 610-639. 
38. Frisz, J. F.; Lou, K.; Klitzing, H. A.; Hanafin, W. P.; Lizunov, V.; Wilson, R. L.; Carpenter, K. J.; Kim, R.; Hutcheon, I. D.; Zimmerberg, J., Direct Chemical Evidence for Sphingolipid Domains in the Plasma Membranes of Fibroblasts. Proc. Natl. Acad. Sci.USA 2013, 110, E613-E622.

39. van Hove, E. R. A.; Smith, D. F.; Heeren, R. M., A Concise Review of Mass Spectrometry Imaging. J. Chromatogr. 2010, 1217, 3946-3954.

40. Heeren, R. M., Getting the Picture: The Coming of Age of Imaging MS. Int. J. Mass Spectrom. 2015, 377, 672-680.

41. Winograd, N., Ion Beams and Laser Postionization for Molecule-Specific Imaging. Anal. Chem. 1993, 65, 622A-629A.

42. Ellis, S. R.; Bruinen, A. L.; Heeren, R. M., A Critical Evaluation of the Current State-ofthe-Art in Quantitative Imaging Mass Spectrometry. Anal. Bioanal. Chem. 2014, 406, 1275-1289.

43. Vickerman, J. C., Molecular Imaging and Depth Profiling by Mass Spectrometry-SIMS, MALDI or DESI? Analyst 2011, 136, 2199-2217.

44. Vickerman, J. C.; Winograd, N., SIMS - a Precursor and Partner to Contemporary Mass Spectrometry. Int. J. Mass spectrom. 2015, 377, 568-579.

45. Vickerman, J. C.; Briggs, D., TOF-SIMS: Materials Analysis by Mass Spectrometry; im publications, 2013.

46. Bich, C.; Touboul, D.; Brunelle, A., Cluster TOF-SIMS Imaging as a Tool for Micrometric Histology of Lipids in Tissue. Mass Spectrom. Rev. 2014, 33, 442-451.

47. Brunelle, A.; Laprévote, O., Lipid Imaging with Cluster Time-of-Flight Secondary Ion Mass Spectrometry. Anal. Bioanal. Chem. 2009, 393, 31-35.

48. Passarelli, M. K.; Winograd, N., Lipid Imaging with Time-of-Flight Secondary Ion Mass Spectrometry (TOF-SIMS). Biochim. Biophys. Acta 2011, 1811, 976-990.

49. Prideaux, B.; Stoeckli, M., Mass Spectrometry Imaging for Drug Distribution Studies. J. Proteomics 2012, 75, 4999-5013.

50. Russo, R. E.; Mao, X.; Liu, H.; Gonzalez, J.; Mao, S. S., Laser Ablation in Analytical Chemistry-a Review. Talanta 2002, 57, 425-451. 
51. Russo, R. E.; Mao, X.; Gonzalez, J. J.; Zorba, V.; Yoo, J., Laser Ablation in Analytical Chemistry. Anal. Chem. 2013, 85, 6162-6177.

52. Vogel, A.; Venugopalan, V., Mechanisms of Pulsed Laser Ablation of Biological Tissues. Chem. Rev. 2003, 103, 577-644.

53. Fang-Yen, C.; Gabel, C. V.; Samuel, A. D.; Bargmann, C. I.; Avery, L., Laser Microsurgery in Caenorhabditis Elegans. Methods Cell Biol. 2012, 107, 177.

54. Kagerer, M.; Irlinger, F.; Lueth, T. C. In Laser Source Independent Basic ParametersFocus Position, Pulse Overlap, Track Overlap-in Laser Micro Milling Using as Rapid Manufacturing Process, Advanced Intelligent Mechatronics (AIM), 2012 IEEE/ASME International Conference on, IEEE: 2012; pp 135-140.

55. Rodríguez, A.; Arriola, A.; Tavera, T.; Pérez, N.; Olaizola, S. M., Enhanced Depth Control of Ultrafast Laser Micromachining of Microchannels in Soda-Lime Glass. Microelectron. Eng. 2012, 98, 672-675.

56. Chrisey, D.; Pique, A.; McGill, R.; Horwitz, J.; Ringeisen, B.; Bubb, D.; Wu, P., Laser Deposition of Polymer and Biomaterial Films. Chem. Rev. 2003, 103, 553-576.

57. Vertes, A., Soft Laser Desorption Ionization-MALDI, DIOS and Nanostructures. In Laser Ablation and Its Applications, Springer: 2007; pp 505-528.

58. Dreisewerd, K., The Desorption Process in MALDI. Chem. Rev. 2003, 103, 395-426.

59. Haglund, R. F.; Miller, J. C.; Lucatorto, T.; De Graef, M., Laser Ablation and Desorption; Academic Press, 1997; Vol. 30.

60. Miller, J. C.(Ed), Laser Ablation: Principles and Applications; Springer Science \& Business Media, 2013; Vol. 28.

61. Hillenkamp, F.; Unsöld, E.; Kaufmann, R.; Nitsche, R., A High-Sensitivity Laser Microprobe Mass Analyzer. Appl. Phys. 1975, 8, 341-348.

62. Hillenkamp, F.; UNSöLD, E.; Kaufmann, R.; Nitshe, R., Laser Microprobe Mass Analysis of Organic Materials. Nature 1975, 256, 119-120.

63. Feigl, P.; Schueler, B.; Hillenkamp, F., Lamma 1000, a New Instrument for Bulk Microprobe Mass Analysis by Pulsed Laser Irradiation. Int. J. Mass Spectrom. Ion Phys. 1983, 47, 15-18. 
64. Holm, R.; Kaempf, G.; Kirchner, D.; Heinen, H.; Meier, S., Laser Microprobe Mass Analysis of Condensed Matter under Atmospheric Conditions. Anal. Chem. 1984, 56, 690-692.

65. Abbe, E., Beiträge Zur Theorie Des Mikroskops Und Der Mikroskopischen Wahrnehmung. Arch. Mikrosk. Anat. 1873, 9, 413-418.

66. Dunn, R. C., Near-Field Scanning Optical Microscopy. Chem. Rev. 1999, 99, 2891-2928.

67. Novotny, L.; Stranick, S. J., Near-Field Optical Microscopy and Spectroscopy with Pointed Probes. Annu. Rev. Phys. Chem. 2006, 57, 303-331.

68. Rasmussen, A.; Deckert, V., New Dimension in Nano-Imaging: Breaking through the Diffraction Limit with Scanning Near-Field Optical Microscopy. Anal. Bioanal. Chem. 2005, 381, 165-172.

69. Zavalin, A.; Yang, J.; Caprioli, R., Laser Beam Filtration for High Spatial Resolution MALDI Imaging Mass Spectrometry. J. Am. Soc. Mass Spectrom. 2013, 24, 1153-1156.

70. Török, P.; Kao, F.-J., Optical Imaging and Microscopy: Techniques and Advanced Systems; Springer Science \& Business Media, 2003; Vol. 87.

71. Siegman, A. E. In Defining, Measuring, and Optimizing Laser Beam Quality, OE/LASE'93: Optics, Electro-Optics, \& Laser Applications in Science \& Engineering, International Society for Optics and Photonics: 1993; pp 2-12.

72. Van Vaeck, L.; Gijbels, R., Laser Microprobe Mass Spectrometry: Potential and Limitations for Inorganic and Organic Micro-Analysis. Fresenius J. Anal. Chem. 1990, 337, 743-754.

73. Spengler, B.; Karas, M.; Bahr, U.; Hillenkamp, F., Laser Mass Analysis in Biology. Ber. Bunsenge. Phys. Chem. 1989, 93, 396-402.

74. Mathey, A.; Van Vaeck, L.; Steglich, W., Investigation of Semi-Thin Cryosections of Lichens by Laser Microprobe Mass Spectrometry. Anal. Chim. Acta 1987, 195, 89-96.

75. Verbueken, A.; Van Grieken, R.; De Broe, M.; Wedeen, R., Identification of Inorganic and Organic Microliths in Kidney Sections by Laser Microprobe Mass Spectrometry. Anal. Chim. Acta 1987, 195, 97-115.

76. Trevor, J. L.; Lykke, K. R.; Pellin, M. J.; Hanley, L., Two-Laser Mass Spectrometry of Thiolate, Disulfide, and Sulfide Self-Assembled Monolayers. Langmuir 1998, 14, 16641673. 
77. Savina, M. R.; Lykke, K. R., Microscopic Chemical Imaging with Laser Desorption Mass Spectrometry. Anal. Chem. 1997, 69, 3741-3746.

78. Behm, J.; Hemminger, J.; Lykke, K. R., Microscopic Laser Desorption/Postionization Fourier Transform Mass Spectrometry. Anal. Chem. 1996, 68, 713-719.

79. Spraggins, J. M.; Caprioli, R. M., High-Speed MALDI-TOF Imaging Mass Spectrometry: Rapid Ion Image Acquisition and Considerations for Next Generation Instrumentation. J. Am. Soc. Mass Spectrom. 2011, 22, 1022-1031.

80. Gessel, M. M.; Norris, J. L.; Caprioli, R. M., MALDI Imaging Mass Spectrometry: Spatial Molecular Analysis to Enable a New Age of Discovery. J. Proteomics 2014, 1-12.

81. Passarelli, M. K.; Wang, J.; Mohammadi, A. S.; Trouillon, R.; Gilmore, I.; Ewing, A. G., Development of an Organic Lateral Resolution Test Device for Imaging Mass Spectrometry. Anal. Chem. 2014, 86, 9473-9480.

82. Yang, J.; Caprioli, R. M., Matrix Sublimation/Recrystallization for Imaging Proteins by Mass Spectrometry at High Spatial Resolution. Anal. Chem. 2011, 83, 5728-5734.

83. Dufresne, M.; Thomas, A.; Breault-Turcot, J.; Masson, J.-F.; Chaurand, P., SilverAssisted Laser Desorption Ionization for High Spatial Resolution Imaging Mass Spectrometry of Olefins from Thin Tissue Sections. Anal. Chem. 2013, 85, 3318-3324.

84. Jurchen, J. C.; Rubakhin, S. S.; Sweedler, J. V., MALDI-MS Imaging of Features Smaller Than the Size of the Laser Beam. J. Am. Soc. Mass Spectrom. 2005, 16, 16541659.

85. Thiery-Lavenant, G.; Zavalin, A. I.; Caprioli, R. M., Targeted Multiplex Imaging Mass Spectrometry in Transmission Geometry for Subcellular Spatial Resolution. J. Am. Soc. Mass Spectrom. 2013, 24, 609-614.

86. Dreisewerd, K.; Schuerenberg, M.; Karas, M.; Hillenkamp, F., Influence of the Laser Intensity and Spot Size on the Desorption of Molecules and Ions in Matrix-Assisted Laser Desorption/Ionization with a Uniform Beam Profile. Int. J. Mass. Spectrom. Ion Processes 1995, 141, 127-148.

87. Qiao, H.; Spicer, V.; Ens, W., The Effect of Laser Profile, Fluence, and Spot Size on Sensitivity in Orthogonal-Injection Matrix-Assisted Laser Desorption/Ionization Timeof-Flight Mass Spectrometry. Rapid. Commun. Mass Spectrom. 2008, 22, 2779-2790. 
88. Zavalin, A.; Yang, J.; Haase, A.; Holle, A.; Caprioli, R., Implementation of a Gaussian Beam Laser and Aspheric Optics for High Spatial Resolution MALDI Imaging MS. $J$. Am. Soc. Mass Spectrom. 2014, 25, 1079-1082.

89. Kettling, H.; Vens-Cappell, S.; Soltwisch, J.; Pirkl, A.; Haier, J. r.; Müthing, J.; Dreisewerd, K., MALDI Mass Spectrometry Imaging of Bioactive Lipids in Mouse Brain with a Synapt G2-S Mass Spectrometer Operated at Elevated Pressure: Improving the Analytical Sensitivity and the Lateral Resolution to Ten Micrometers. Anal. Chem. 2014, 86, 7798-7805.

90. Korte, A. R.; Yandeau-Nelson, M. D.; Nikolau, B. J.; Lee, Y. J., Subcellular-Level Resolution MALDI-MS Imaging of Maize Leaf Metabolites by MALDI-Linear Ion TrapOrbitrap Mass Spectrometer. Anal. Bioanal. Chem. 2015, 407, 2301-2309.

91. Thiery-Lavenant, G.; Zavalin, A. I.; Caprioli, R. M., Targeted Multiplex Imaging Mass Spectrometry in Transmission Geometry for Subcellular Spatial Resolution. J. Am. Soc. Mass Spectrom. 2013, 24, 609-614.

92. Zavalin, A.; Todd, E. M.; Rawhouser, P. D.; Yang, J.; Norris, J. L.; Caprioli, R. M., Direct Imaging of Single Cells and Tissue at Sub - Cellular Spatial Resolution Using Transmission Geometry MALDI MS. J. Mass Spectrom. 2012, 47, 1473-1481.

93. Zavalin, A.; Yang, J.; Hayden, K.; Vestal, M.; Caprioli, R. M., Tissue Protein Imaging at $1 \mathrm{Mm}$ Laser Spot Diameter for High Spatial Resolution and High Imaging Speed Using Transmission Geometry MALDI TOF MS. Anal. Bioanal. Chem. 2015, 407, 2337-2342.

94. Luxembourg, S. L.; McDonnell, L. A.; Mize, T. H.; Heeren, R. M., Infrared Mass Spectrometric Imaging Below the Diffraction Limit. J. Proteome Res. 2005, 4, 671-673.

95. Römpp, A.; Spengler, B., Mass Spectrometry Imaging with High Resolution in Mass and Space. Histochem. Cell Biol. 2013, 139, 759-783.

96. Spengler, B.; Hubert, M., Scanning Microprobe Matrix-Assisted Laser Desorption Ionization (SMALDI) Mass Spectrometry: Instrumentation for Sub-Micrometer Resolved LDI and MALDI Surface Analysis. J. Am. Soc. Mass Spectrom. 2002, 13, 735-748.

97. Bouschen, W.; Spengler, B., Artifacts of MALDI Sample Preparation Investigated by High-Resolution Scanning Microprobe Matrix-Assisted Laser Desorption/Ionization (SMALDI) Imaging Mass Spectrometry. Int. J. Mass Spectrom. 2007, 266, 129-137.

98. Koestler, M.; Kirsch, D.; Hester, A.; Leisner, A.; Guenther, S.; Spengler, B., A HighResolution Scanning Microprobe Matrix-Assisted Laser Desorption/Ionization Ion 
Source for Imaging Analysis on an Ion Trap/Fourier Transform Ion Cyclotron Resonance Mass Spectrometer. Rapid Commun. Mass Spectrom. 2008, 22, 3275-3285.

99. Bouschen, W.; Schulz, O.; Eikel, D.; Spengler, B., Matrix Vapor Deposition/Recrystallization and Dedicated Spray Preparation for High - Resolution Scanning Microprobe Matrix - Assisted Laser Desorption/Ionization Imaging Mass Spectrometry (SMALDI - MS) of Tissue and Single Cells. Rapid Commun. Mass Spectrom. 2010, 24, 355-364.

100. Römpp, A.; Both, J.-P.; Brunelle, A.; Heeren, R. M.; Laprévote, O.; Prideaux, B.; Seyer, A.; Spengler, B.; Stoeckli, M.; Smith, D. F., Mass Spectrometry Imaging of Biological Tissue: An Approach for Multicenter Studies. Anal. Bioanal. Chem. 2015, 407, 23292335.

101. Cooks, R. G.; Ouyang, Z.; Takats, Z.; Wiseman, J., Detection Technologies. Ambient Mass Spectrometry. Science 2006, 311, 1566-1570.

102. Huang, M.; Yuan, C.; Cheng, S.; Cho, Y.; Shiea, J., Ambient Ionization Mass Spectrometry. Annu. Rev. Anal. Chem. 2010, 3, 43-65.

103. Wu, C.; Dill, A. L.; Eberlin, L. S.; Cooks, R. G.; Ifa, D. R., Mass Spectrometry Imaging under Ambient Conditions. Mass Spectrom. Rev. 2013, 32, 218-243.

104. Hsu, C.-C.; Dorrestein, P. C., Visualizing Life with Ambient Mass Spectrometry. Curr. Opin. Biotechnol. 2014, 31C, 24-34.

105. Van Berkel, G. J.; Pasilis, S. P.; Ovchinnikova, O., Established and Emerging Atmospheric Pressure Surface Sampling/Ionization Techniques for Mass Spectrometry. J. Mass Spectrom. 2008, 43, 1161-1180.

106. Harris, G. A.; Nyadong, L.; Fernandez, F. M., Recent Developments in Ambient Ionization Techniques for Analytical Mass Spectrometry. Analyst 2008, 133, 1297-1301.

107. Harris, G. A.; Galhena, A. S.; Fernández, F. M., Ambient Sampling/Ionization Mass Spectrometry: Applications and Current Trends. Anal. Chem. 2011, 83, 4508-4538.

108. Monge, M. E.; Harris, G. A.; Dwivedi, P.; Fernández, F. M., Mass Spectrometry: Recent Advances in Direct Open Air Surface Sampling/Ionization. Chem. Rev. 2013, 113, 22692308.

109. McEwen, C. N.; Larsen, B. S., Fifty Years of Desorption Ionization of Nonvolatile Compounds. Int. J. Mass Spectrom. 2015, 377, 515-531. 
110. Takats, Z.; Wiseman, J. M.; Gologan, B.; Cooks, R. G., Mass Spectrometry Sampling under Ambient Conditions with Desorption Electrospray Ionization. Science 2004, 306, 471-473.

111. Takats, Z.; Wiseman, J. M.; Cooks, R. G., Ambient Mass Spectrometry Using Desorption Electrospray Ionization (DESI): Instrumentation, Mechanisms and Applications in Forensics, Chemistry, and Biology. J. Mass Spectrom. 2005, 40, 1261-1275.

112. Wu, C.; Dill, A. L.; Eberlin, L. S.; Cooks, R. G.; Ifa, D. R., Mass Spectrometry Imaging under Ambient Conditions. Mass Spectrom. Rev. 2013, 32, 218-243.

113. Wiseman, J. M.; Puolitaival, S. M.; Takáts, Z.; Cooks, R. G.; Caprioli, R. M., Mass Spectrometric Profiling of Intact Biological Tissue by Using Desorption Electrospray Ionization. Angew. Chem. 2005, 117, 7256-7259.

114. Eberlin, L. S.; Ifa, D. R.; Wu, C.; Cooks, R. G., Three - Dimensional Vizualization of Mouse Brain by Lipid Analysis Using Ambient Ionization Mass Spectrometry. Angew. Chem. Int. Ed. 2010, 49, 873-876.

115. Calligaris, D.; Caragacianu, D.; Liu, X.; Norton, I.; Thompson, C. J.; Richardson, A. L.; Golshan, M.; Easterling, M. L.; Santagata, S.; Dillon, D. A., Application of Desorption Electrospray Ionization Mass Spectrometry Imaging in Breast Cancer Margin Analysis. Proc. Natl. Acad. Sci.USA 2014, 111, 15184-15189.

116. Campbell, D. I.; Ferreira, C. R.; Eberlin, L. S.; Cooks, R. G., Improved Spatial Resolution in the Imaging of Biological Tissue Using Desorption Electrospray Ionization. Anal. Bioanal. Chem. 2012, 404, 389-398.

117. Wiseman, J. M.; Ifa, D. R.; Venter, A.; Cooks, R. G., Ambient Molecular Imaging by Desorption Electrospray Ionization Mass Spectrometry. Nat. Protoc. 2008, 3, 517-524.

118. Laskin, J.; Heath, B. S.; Roach, P. J.; Cazares, L.; Semmes, O. J., Tissue Imaging Using Nanospray Desorption Electrospray Ionization Mass Spectrometry. Anal. Chem. 2011, $84,141-148$.

119. Roach, P. J.; Laskin, J.; Laskin, A., Nanospray Desorption Electrospray Ionization: An Ambient Method for Liquid-Extraction Surface Sampling in Mass Spectrometry. Analyst 2010, 135, 2233-2236.

120. Roach, P. J.; Laskin, J.; Laskin, A., Molecular Characterization of Organic Aerosols Using Nanospray-Desorption/Electrospray Ionization-Mass Spectrometry. Anal. Chem. 2010, 82, 7979-7986. 
121. Van Berkel, G. J.; Kertesz, V.; King, R. C., High-Throughput Mode Liquid Microjunction Surface Sampling Probe. Anal. Chem. 2009, 81, 7096-7101.

122. Kertesz, V.; Van Berkel, G. J., Fully Automated Liquid Extraction - Based Surface Sampling and Ionization Using a Chip - Based Robotic Nanoelectrospray Platform. $J$. Mass Spectrom. 2010, 45, 252-260.

123. Rao, W.; Pan, N.; Yang, Z., High Resolution Tissue Imaging Using the Single-Probe Mass Spectrometry under Ambient Conditions. J. Am. Soc. Mass Spectrom. 2015, 26, 986-993.

124. Van Berkel, G. J.; Kertesz, V.; Koeplinger, K. A.; Vavrek, M.; Kong, A. N. T., Liquid Microjunction Surface Sampling Probe Electrospray Mass Spectrometry for Detection of Drugs and Metabolites in Thin Tissue Sections. J. Mass Spectrom. 2008, 43, 500-508.

125. Pan, N.; Rao, W.; Kothapalli, N. R.; Liu, R.; Burgett, A. W.; Yang, Z., The Single-Probe: A Miniaturized Multifunctional Device for Single Cell Mass Spectrometry Analysis. Anal. Chem. 2014, 86, 9376-9380.

126. Laskin, J.; Lanekoff, I., Ambient Mass Spectrometry Imaging Using Direct Liquid Extraction Techniques. Anal. Chem. 2015.

127. Hsu, C.-C.; Chou, P.-T.; Zare, R. N., Imaging of Proteins in Tissue Samples Using Nanospray Desorption Electrospray Ionization Mass Spectrometry. Anal. Chem. 2015, 87, 11171-11175.

128. Phelps, M. S.; Verbeck, G. F., A Lipidomics Demonstration of the Importance of Single Cell Analysis. Anal. Methods 2015, 7, 3668-3670.

129. Nemes, P.; Rubakhin, S. S.; Aerts, J. T.; Sweedler, J. V., Qualitative and Quantitative Metabolomic Investigation of Single Neurons by Capillary Electrophoresis Electrospray Ionization Mass Spectrometry. Nat. Protoc. 2013, 8, 783-799.

130. Gong, X.; Zhao, Y.; Cai, S.; Fu, S.; Yang, C.; Zhang, S.; Zhang, X., Single Cell Analysis with Probe Esi-Mass Spectrometry: Detection of Metabolites at Cellular and Subcellular Levels. Anal. Chem. 2014, 86, 3809-3816.

131. Chernetsova, E. S.; Morlock, G. E.; Revelsky, I. A., DART Mass Spectrometry and Its Applications in Chemical Analysis. Russ. Chem. Rev. 2011, 80, 235.

132. Gross, J. H., Direct Analysis in Real Time-a Critical Review on DART-MS. Anal. Bioanal. Chem. 2014, 406, 63-80. 
133. Cody, R. B.; Laramée, J. A.; Durst, H. D., Versatile New Ion Source for the Analysis of Materials in Open Air under Ambient Conditions. Anal. Chem. 2005, 77, 2297-2302.

134. Chernetsova, E. S.; Morlock, G. E., Ambient Desorption Ionization Mass Spectrometry (DART, DESI) and Its Bioanalytical Applications. Bioanal. Rev. 2011, 3, 1-9.

135. Sisco, E.; Dake, J.; Bridge, C., Screening for Trace Explosives by AccuTOFTM_DART®: An in-Depth Validation Study. Forensic Sci. Int. 2013, 232, 160-168.

136. Badu-Tawiah, A. K.; Eberlin, L. S.; Ouyang, Z.; Cooks, R. G., Chemical Aspects of the Extractive Methods of Ambient Ionization Mass Spectrometry. Annu. Rev. Phys. Chem. 2013, 64, 481-505.

137. Na, N.; Zhao, M.; Zhang, S.; Yang, C.; Zhang, X., Development of a Dielectric Barrier Discharge Ion Source for Ambient Mass Spectrometry. J. Am. Soc. Mass Spectrom. 2007, $18,1859-1862$.

138. Ratcliffe, L. V.; Rutten, F. J.; Barrett, D. A.; Whitmore, T.; Seymour, D.; Greenwood, C.; Aranda-Gonzalvo, Y.; Robinson, S.; McCoustra, M., Surface Analysis under Ambient Conditions Using Plasma-Assisted Desorption/Ionization Mass Spectrometry. Anal. Chem. 2007, 79, 6094-6101.

139. McEwen, C. N.; McKay, R. G.; Larsen, B. S., Analysis of Solids, Liquids, and Biological Tissues Using Solids Probe Introduction at Atmospheric Pressure on Commercial LC/MS Instruments. Anal. Chem. 2005, 77, 7826-7831.

140. Andrade, F. J.; Shelley, J. T.; Wetzel, W. C.; Webb, M. R.; Gamez, G.; Ray, S. J.; Hieftje, G. M., Atmospheric Pressure Chemical Ionization Source. 2. DesorptionIonization for the Direct Analysis of Solid Compounds. Anal. Chem. 2008, 80, 26542663.

141. Dill, A. L.; Eberlin, L. S.; Ifa, D. R.; Cooks, R. G., Perspectives in Imaging Using Mass Spectrometry. Chem. Comm. 2011, 47, 2741-2746.

142. Nemes, P.; Vertes, A., Laser Ablation Electrospray Ionization for Atmospheric Pressure Molecular Imaging Mass Spectrometry. Meth. Mol. Biol. 2010, 656, 159-171.

143. Shelley, J. T.; Ray, S. J.; Hieftje, G. M., Laser Ablation Coupled to a Flowing Atmospheric Pressure Afterglow for Ambient Mass Spectral Imaging. Anal. Chem. 2008, 80, 8308-8313.

144. Shrestha, B.; Vertes, A., In Situ Metabolic Profiling of Single Cells by Laser Ablation Electrospray Ionization Mass Spectrometry. Anal. Chem. 2009, 81, 8265-8271. 
145. Laiko, V. V.; Baldwin, M. A.; Burlingame, A. L., Atmospheric Pressure Matrix-Assisted Laser Desorption/Ionization Mass Spectrometry. Anal. Chem. 2000, 72, 652-657.

146. Laiko, V. V.; Taranenko, N. I.; Berkout, V. D.; Yakshin, M. A.; Prasad, C. R.; Lee, H. S.; Doroshenko, V. M., Desorption/Ionization of Biomolecules from Aqueous Solutions at Atmospheric Pressure Using an Infrared Laser at $3 \mathrm{Mm}$. J. Am. Soc. Mass Spectrom. 2002, 13, 354-361.

147. Li, Y.; Shrestha, B.; Vertes, A., Atmospheric Pressure Molecular Imaging by Infrared MALDI Mass Spectrometry. Anal. Chem. 2007, 79, 523-32.

148. Shiea, J.; Huang, M.; Hsu, H.; Lee, C.; Yuan, C.; Beech, I.; Sunner, J., ElectrosprayAssisted Laser Desorption/Ionization Mass Spectrometry for Direct Ambient Analysis of Solids. Rapid Commun. Mass Spectrom. 2005, 19, 3701-3704.

149. Gray, A. L., Solid Sample Introduction by Laser Ablation for Inductively Coupled Plasma Source Mass Spectrometry. Analyst 1985, 110, 551-556.

150. Russo, R. E.; Mao, X.; Liu, H.; Gonzalez, J.; Mao, S., Laser Ablation in Analytical Chemistry-a Review. Talanta 2002.

151. Russo, R. E.; Mao, X.; Gonzalez, J. J.; Zorba, V.; Yoo, J., Laser Ablation in Analytical Chemistry. Anal. Chem. 2013, 85, 6162-6177.

152. Coon, J. J.; McHale, K. J.; Harrison, W. W., Atmospheric Pressure Laser Desorption/Chemical Ionization Mass Spectrometry: A New Ionization Method Based on Existing Themes. Rapid Commun. Mass Spectrom. 2002, 16, 681-685.

153. Nyadong, L.; Galhena, A. S.; Fernandez, F. M., Desorption Electrospray/MetastableInduced Ionization: A Flexible Multimode Ambient Ion Generation Technique. Anal. Chem. 2009, 81, 7788-7794.

154. Brady, J. J.; Judge, E. J.; Levis, R. J., Mass Spectrometry of Intact Neutral Macromolecules Using Intense Non-Resonant Femtosecond Laser Vaporization with Electrospray Post-Ionization. Rapid Commun. Mass Spectrom. 2009, 23, 3151-3157.

155. Sampson, J.; Hawkridge, A. M.; Muddiman, D. C., Generation and Detection of Multiply-Charged Peptides and Proteins by Matrix-Assisted Laser Desorption Electrospray Ionization (MALDESI) Fourier Transform Ion Cyclotron Resonance Mass Spectrometry. J. Am. Soc. Mass Spectrom. 2006, 17, 1712-1716. 
156. Vaikkinen, A.; Shrestha, B.; Kauppila, T. J.; Vertes, A.; Kostiainen, R., Infrared Laser Ablation Atmospheric Pressure Photoionization Mass Spectrometry. Anal. Chem. 2012, 84, 1630-1636.

157. Cramer, R.(Ed), Advances in MALDI and Laser-Induced Soft Ionization Mass Spectrometry; Springer, 2015.

158. Galhena, A. S.; Harris, G. A.; Nyadong, L.; Murray, K. K.; Fernández, F. M., Small Molecule Ambient Mass Spectrometry Imaging by Infrared Laser Ablation MetastableInduced Chemical Ionization. Anal. Chem. 2010, 82, 2178-2181.

159. Razunguzwa, T. T.; Henderson, H. D.; Reschke, B. R.; Walsh, C. M.; Powell, M. J., Laser-Ablation Electrospray Ionization Mass Spectrometry (LAESIS-MS): Ambient Ionization Technology for 2D and 3D Molecular Imaging. Ambient ionization mass spectrometry. Royal Society of Chemistry, Cambridge 2014, 462-481.

160. Nielen, M. W.; van Beek, T. A., Macroscopic and Microscopic Spatially-Resolved Analysis of Food Contaminants and Constituents Using Laser-Ablation Electrospray Ionization Mass Spectrometry Imaging. Anal. Bioanal. Chem. 2014, 406, 6805-6815.

161. Li, H.; Smith, B. K.; Márk, L.; Nemes, P.; Nazarian, J.; Vertes, A., Ambient Molecular Imaging by Laser Ablation Electrospray Ionization Mass Spectrometry with Ion Mobility Separation. Int. J. Mass Spectrom. 2015, 377, 681-689.

162. Shrestha, B.; Nemes, P.; Nazarian, J.; Hathout, Y.; Hoffman, E. P.; Vertes, A., Direct Analysis of Lipids and Small Metabolites in Mouse Brain Tissue by AP IR-MALDI and Reactive Laesi Mass Spectrometry. Analyst 2010, 135, 751-758.

163. Li, H.; Smith, B. K.; Shrestha, B.; Márk, L.; Vertes, A., Automated Cell-by-Cell Tissue Imaging and Single-Cell Analysis for Targeted Morphologies by Laser Ablation Electrospray Ionization Mass Spectrometry. In Mass Spectrometry Imaging of Small Molecules He, L., Ed. Springer New York: New York, NY, 2015; pp 117-127.

164. Nazari, M.; Muddiman, D. C., MALDESI: Fundamentals, Direct Analysis, and MS Imaging. In Advances in MALDI and Laser-Induced Soft Ionization Mass Spectrometry, Springer: 2016; pp 169-182.

165. Dixon, R. B.; Muddiman, D. C., Study of the Ionization Mechanism in Hybrid Laser Based Desorption Techniques. Analyst 2010, 135, 880-882.

166. Barry, J. A.; Robichaud, G.; Bokhart, M. T.; Thompson, C.; Sykes, C.; Kashuba, A. D.; Muddiman, D. C., Mapping Antiretroviral Drugs in Tissue by IR-MALDESI MSI 
Coupled to the Q Exactive and Comparison with LC-MS/MS SRM Assay. J. Am. Soc. Mass Spectrom. 2014, 25, 2038-2047.

167. Cochran, K. H.; Barry, J. A.; Muddiman, D. C.; Hinks, D., Direct Analysis of Textile Fabrics and Dyes Using Infrared Matrix-Assisted Laser Desorption Electrospray Ionization Mass Spectrometry. Anal. Chem. 2012, 85, 831-836.

168. Nazari, M.; Muddiman, D. C., Cellular-Level Mass Spectrometry Imaging Using Infrared Matrix-Assisted Laser Desorption Electrospray Ionization (IR-MALDESI) by Oversampling. Anal. Bioanal. Chem. 2015, 407, 2265-2271.

169. Robichaud, G.; Barry, J. A.; Garrard, K. P.; Muddiman, D. C., Infrared Matrix-Assisted Laser Desorption Electrospray Ionization (IR-MALDESI) Imaging Source Coupled to a Ft-Icr Mass Spectrometer. J. Am. Soc. Mass Spectrom. 2013, 24, 92-100.

170. Guenther, S.; Römpp, A.; Kummer, W.; Spengler, B., AP-MALDI Imaging of Neuropeptides in Mouse Pituitary Gland with $5 \mu \mathrm{m}$ Spatial Resolution and High Mass Accuracy. Int. J. Mass Spectrom. 2011, 305, 228-237.

171. Khalil, S. M.; Römpp, A.; Pretzel, J.; Becker, K.; Spengler, B., Phospholipid Topography of Whole-Body Sections of the Anopheles Stephensi Mosquito, Characterized by HighResolution Atmospheric-Pressure Scanning Microprobe Matrix-Assisted Laser Desorption/Ionization Mass Spectrometry Imaging. Anal. Chem. 2015, 87, 11309-11316.

172. Ovchinnikova, O. S.; Kertesz, V.; Van Berkel, G. J., Combining Laser Ablation/Liquid Phase Collection Surface Sampling and High-Performance Liquid ChromatographyElectrospray Ionization-Mass Spectrometry. Anal. Chem. 2011, 83, 1874-1878.

173. Ovchinnikova, O. S.; Kertesz, V.; Van Berkel, G. J., Combining Transmission Geometry Laser Ablation and a Non-Contact Continuous Flow Surface Sampling Probe/Electrospray Emitter for Mass Spectrometry Based Chemical Imaging. Rapid Commun. Mass Spectrom. 2011, 25, 3735-3740.

174. Cahill, J. F.; Kertesz, V.; Van Berkel, G. J., Characterization and Application of a Hybrid Optical Microscopy/Laser Ablation Liquid Vortex Capture/Electrospray Ionization System for Mass Spectrometry Imaging with Sub-Micrometer Spatial Resolution. Anal. Chem. 2015, 87, 11113-11121.

175. Park, S. G.; Murray, K. K., Ambient Laser Ablation Sampling for Capillary Electrophoresis Mass Spectrometry. Rapid Commun. Mass Spectrom. 2013, 27, 16731680. 
176. Park, S. G.; Murray, K. K., Infrared Laser Ablation Sample Transfer for MALDI and Electrospray. J. Am. Soc. Mass Spectrom. 2011, 22, 1352-1362.

177. Park, S. G.; Murray, K. K., Infrared Laser Ablation Sample Transfer for on-Line Liquid Chromatography Electrospray Ionization Mass Spectrometry. J. Mass Spectrom. 2012, 47, 1322-1326.

178. Park, S. G.; Murray, K. K., Infrared Laser Ablation Sample Transfer for MALDI Imaging. Anal. Chem. 2012, 84, 3240-3245.

179. Cleveland, D.; Michel, R. G., A Review of Near - Field Laser Ablation for High Resolution Nanoscale Surface Analysis. Appl. Spectrosc. Rev. 2008, 43, 93-110.

180. Novotny, L., The History of Near-Field Optics. Prog. Optics 2007, 50, 137-184.

181. Synge, E., Xxxviii. A Suggested Method for Extending Microscopic Resolution into the Ultra-Microscopic Region. Lond. Edinbur. Dub. Philos. Mag. J. Sci. 1928, 6, 356-362.

182. Pohl, D. W.; Courjon, D., Near Field Optics; Springer Science \& Business Media, 2012; Vol. 242.

183. De Angelis, F.; Das, G.; Candeloro, P.; Patrini, M.; Galli, M.; Bek, A.; Lazzarino, M.; Maksymov, I.; Liberale, C.; Andreani, L. C., Nanoscale Chemical Mapping Using ThreeDimensional Adiabatic Compression of Surface Plasmon Polaritons. Nature Nanotechnology 2010, 5, 67-72.

184. Wallace, J. M., Applications of Atomic Force Microscopy for the Assessment of Nanoscale Morphological and Mechanical Properties of Bone. Bone 2012, 50, 420-427.

185. Shan, Y.; Wang, H., The Structure and Function of Cell Membranes Examined by Atomic Force Microscopy and Single-Molecule Force Spectroscopy. Chem. Soc. Rev. 2015, 44, 3617-3638.

186. Müller, D. J.; Dufrêne, Y. F., Atomic Force Microscopy: A Nanoscopic Window on the Cell Surface. Trend. Cell Biol. 2011, 21, 461-469.

187. Morris, V. J.; Kirby, A. R.; Gunning, A. P., Atomic Force Microscopy for Biologists; World Scientific, 1999; Vol. 57.

188. Kurland, N. E.; Drira, Z.; Yadavalli, V. K., Measurement of Nanomechanical Properties of Biomolecules Using Atomic Force Microscopy. Micron 2012, 43, 116-128. 
189. Binnig, G.; Quate, C. F.; Gerber, C., Atomic Force Microscope. Phys. Rev. Lett. 1986, $56,930$.

190. Tersoff, J.; Hamann, D., Theory and Application for the Scanning Tunneling Microscope. Phys. Rev. Lett. 1983, 50, 1998.

191. Baró, A. M.; Reifenberger, R. G., Atomic Force Microscopy in Liquid: Biological Applications; John Wiley \& Sons, 2012.

192. Foster, A.; Hofer, W. A., Scanning Probe Microscopy: Atomic Scale Engineering by Forces and Currents; Springer Science \& Business Media, 2006.

193. Meyer, G.; Amer, N. M., Novel Optical Approach to Atomic Force Microscopy. Appl. Phys. Lett. 1988, 53, 1045-1047.

194. Cappella, B.; Dietler, G., Force-Distance Curves by Atomic Force Microscopy. Surf. Sci. Rep. 1999, 34, 1-104.

195. Salapaka, S. M.; Salapaka, M. V., Scanning Probe Microscopy. Control Systems, IEEE 2008, 28, 65-83.

196. Wiesendanger, R., Scanning Probe Microscopy: Analytical Methods; Springer Science \& Business Media, 1998.

197. Kalinin, S. V.; Gruverman, A., Scanning Probe Microscopy: Electrical and Electromechanical Phenomena at the Nanoscale; Springer Science \& Business Media, 2007; Vol. 1.

198. Bhushan, B.; Marti, O., Scanning Probe Microscopy-Principle of Operation, Instrumentation, and Probes. In Nanotribology and Nanomechanics, Springer: 2005; pp 41-115.

199. Rajendran, A.; Endo, M.; Sugiyama, H., State-of-the-Art High-Speed Atomic Force Microscopy for Investigation of Single-Molecular Dynamics of Proteins. Chem. Rev. 2013, 114, 1493-1520.

200. Pohl, D. W.; Denk, W.; Lanz, M., Optical Stethoscopy: Image Recording with Resolution 7/20. Appl. Phys. Lett. 1984, 44, 651-653.

201. Tsai, D. P.; Othonos, A.; Moskovits, M.; Uttamchandani, D., Raman Spectroscopy Using a Fiber Optic Probe with Subwavelength Aperture. Appl. Phys. Lett. 1994, 64, 17681770 . 
202. Abramczyk, H.; Brozek-Pluska, B., Raman Imaging in Biochemical and Biomedical Applications. Diagnosis and Treatment of Breast Cancer. Chem. Rev. 2013, 113, 57665781.

203. Schmid, T.; Opilik, L.; Blum, C.; Zenobi, R., Nanoscale Chemical Imaging Using Tip Enhanced Raman Spectroscopy: A Critical Review. Angew. Chem. Int. Ed. 2013, 52, 5940-5954.

204. Wessel, J., Surface-Enhanced Optical Microscopy. JOSA B 1985, 2, 1538-1541.

205. Grober, R. D.; Schoelkopf, R. J.; Prober, D. E., Optical Antenna: Towards a Unity Efficiency Near-Field Optical Probe. Appl. Phys. Lett. 1997, 70, 1354-1356.

206. Olmon, R. L.; Krenz, P. M.; Jones, A. C.; Boreman, G. D.; Raschke, M. B., Near-Field Imaging of Optical Antenna Modes in the Mid-Infrared. Opt. Express 2008, 16, 2029520305.

207. Sánchez, E. J.; Novotny, L.; Xie, X. S., Near-Field Fluorescence Microscopy Based on Two-Photon Excitation with Metal Tips. Phys. Rev. Lett. 1999, 82, 4014.

208. Anderson, M. S., Locally Enhanced Raman Spectroscopy with an Atomic Force Microscope. Appl. Phys. Lett. 2000, 76, 3130-3132.

209. Hayazawa, N.; Inouye, Y.; Sekkat, Z.; Kawata, S., Metallized Tip Amplification of NearField Raman Scattering. Opt. Commun. 2000, 183, 333-336.

210. Kossakovski, D.; O'Connor, S.; Widmer, M.; Baldeschwieler, J.; Beauchamp, J., Spatially Resolved Chemical Analysis with an NSOM-Based Laser Desorption Microprobe. Ultramicroscopy 1998, 71, 111-115.

211. Zeisel, D.; Nettesheim, S.; Dutoit, B.; Zenobi, R., Pulsed Laser - Induced Desorption and Optical Imaging on a Nanometer Scale with Scanning Near - Field Microscopy Using Chemically Etched Fiber Tips. Appl. Phys. Lett. 1996, 68, 2491-2492.

212. Stockle, R.; Setz, P.; Deckert, V.; Lippert, T.; Wokaun, A.; Zenobi, R., Nanoscale Atmospheric Pressure Laser Ablation-Mass Spectrometry. Anal. Chem. 2001, 73, 13991402.

213. Schmitz, T. A.; Gamez, G.; Setz, P. D.; Zhu, L.; Zenobi, R., Towards Nanoscale Molecular Analysis at Atmospheric Pressure by a Near-Field Laser Ablation Ion Trap/Time-of-Flight Mass Spectrometer. Anal. Chem. 2008, 80, 6537-6544. 
214. Zhu, L.; Gamez, G.; Schmitz, T.; Krumeich, F.; Zenobi, R., Material Ejection and Redeposition Following Atmospheric Pressure Near-Field Laser Ablation on Molecular Solids. Anal. Bioanal. Chem. 2010, 396, 163-172.

215. Zhu, L.; Stadler, J.; Schmitz, T. A.; Krumeich, F.; Zenobi, R., Atmospheric Pressure Sampling for Laser Ablation Based Nanoscale Imaging Mass Spectrometry: Ions or Neutrals? J. Phys. Chem. C 2011, 115, 1006-1013.

216. Becker, J. S.; Gorbunoff, A.; Zoriy, M.; Izmer, A.; Kayser, M., Evidence of Near-Field Laser Ablation Inductively Coupled Plasma Mass Spectrometry (NF-LA-ICP-MS) at Nanometre Scale for Elemental and Isotopic Analysis on Gels and Biological Samples. J. Anal. At. Spectrom. 2006, 21, 19-25.

217. Becker, J.; Becker, J. S.; Zoriy, M.; Dobrowolska, J.; Matusch, A., Imaging Mass Spectrometry in Biological Tissues by Laser Ablation Inductively Coupled Plasma Mass Spectrometry. Eur. J. Mass Spectrom. 2007, 13, 1-6.

218. Zoriy, M. V.; Becker, J. S., Near-Field Laser Ablation Inductively Coupled Plasma Mass Spectrometry: A Novel Elemental Analytical Technique at the Nanometer Scale. Rapid Commun. Mass. Spectrom, 2009, 23, 23-30.

219. Wu, B.; Becker, J. S., Imaging of Elements and Molecules in Biological Tissues and Cells in the Low-Micrometer and Nanometer Range. Int. J. Mass Spectrom. 2011, 307, $112-122$.

220. Zoriy, M. V.; Mayer, D.; Becker, J. S., Metal Imaging on Surface of Micro-and Nanoelectronic Devices by Laser Ablation Inductively Coupled Plasma Mass Spectrometry and Possibility to Measure at Nanometer Range. J. Am. Soc. Mass Spectrom. 2009, 20, 883-890.

221. Zoriy, M. V.; Kayser, M.; Becker, J. S., Possibility of Nano-Local Element Analysis by Near-Field Laser Ablation Inductively Coupled Plasma Mass Spectrometry (LA-ICPMS): New Experimental Arrangement and First Application. Int. J. Mass Spectrom. 2008, 273, 151-155.

222. Neacsu, C. C.; Dreyer, J.; Behr, N.; Raschke, M. B., Scanning-Probe Raman Spectroscopy with Single-Molecule Sensitivity. Phys. Rev. B 2006, 73, 193406.

223. Meyer, K. A.; Ovchinnikova, O.; Ng, K.; Goeringer, D. E., Development of a Scanning Surface Probe for Nanoscale Tip-Enhanced Desorption/Ablation. Rev. Sci. Instrum. 2008, $79,123710$. 
224. Bradshaw, J. A.; Ovchinnikova, O. S.; Meyer, K. A.; Goeringer, D. E., Combined Chemical and Topographic Imaging at Atmospheric Pressure Via Microprobe Laser Desorption/Ionization Mass Spectrometry-Atomic Force Microscopy. Rapid Commun. Mass Spectrom. 2009, 23, 3781-3786.

225. Sahagian, K.; Kjoller, K.; Shetty, R. In Rapid Determination of Cure Rate and Direct Identification of Spatial Variations in Cross Link Density, NIP \& Digital Fabrication Conference, Society for Imaging Science and Technology: 2011; pp 409-410.

226. Holle, A.; Haase, A.; Kayser, M.; Hoehndorf, J., Optimizing Uv Laser Focus Profiles for Improved MALDI Performance. J. Mass Spectrom. 2006, 41, 705-716.

227. Carrasco-Pancorbo, A.; Navas-Iglesias, N.; Cuadros-Rodriguez, L., From Lipid Analysis Towards Lipidomics, a New Challenge for the Analytical Chemistry of the 21st Century. Part I: Modern Lipid Analysis. TrAC, Trends Anal. Chem. 2009, 28, 263-278.

228. Matyash, V.; Liebisch, G.; Kurzchalia, T. V.; Shevchenko, A.; Schwudke, D., Lipid Extraction by Methyl-Tert-Butyl Ether for High-Throughput Lipidomics. J. Lipid Res. 2008, 49, 1137-1146.

229. Dill, A. L.; Eberlin, L. S.; Ifa, D. R.; Cooks, R. G., Perspectives in Imaging Using Mass Spectrometry. Chem. Com. 2011, 47, 2741-2746.

230. Yao, Z.-P., Characterization of Proteins by Ambient Mass Spectrometry. Mass Spectrom. Rev. 2011.

231. Ghorai, S.; Seneviratne, C. A.; Murray, K. K., Tip-Enhanced Laser Ablation Sample Transfer for Biomolecule Mass Spectrometry. J. Am. Soc. Mass Spectrom. 2015, 26, 6370.

232. Huang, M.; Jhang, S.; Cheng, C.; Cheng, S.; Shiea, J., Effects of Matrix, Electrospray Solution, and Laser Light on the Desorption and Ionization Mechanisms in ElectrosprayAssisted Laser Desorption Ionization Mass Spectrometry. Analyst 2010, 135, 759-766.

233. Saito, Y.; Hayazawa, N.; Kataura, H.; Murakami, T.; Tsukagoshi, K.; Inouye, Y.; Kawata, S., Polarization Measurements in Tip-Enhanced Raman Spectroscopy Applied to Single-Walled Carbon Nanotubes. Chem. Phys. Lett. 2005, 410, 136-141.

234. Jin, R.; Cao, Y. W.; Mirkin, C. A.; Kelly, K. L.; Schatz, G. C., Photoinduced Conversion of Silver Nanospheres to Nanoprisms. Science 2001. 
235. Sherry, L. J.; Jin, R.; Mirkin, C. A.; Schatz, G. C.; Van Duyne, R. P., Localized Surface Plasmon Resonance Spectroscopy of Single Silver Triangular Nanoprisms. Nano Lett. 2006, 6, 2060-2065.

236. Dickreuter, S.; Gleixner, J.; Kolloch, A.; Boneberg, J.; Scheer, E.; Leiderer, P., Mapping of Plasmonic Resonances in Nanotriangles. Beilstein J. Nanotechnol. 2013, 4, 588-602.

237. Grześkiewicz, B.; Ptaszyński, K.; Kotkowiak, M., Near and Far-Field Properties of Nanoprisms with Rounded Edges. Plasmonics 2014, 9, 607-614.

238. Perdian, D. C.; Cha, S.; Oh, J.; Sakaguchi, D. S.; Yeung, E. S.; Lee, Y. J., In Situ Probing of Cholesterol in Astrocytes at the Single-Cell Level Using Laser Desorption Ionization Mass Spectrometric Imaging with Colloidal Silver. Rapid Commun. Mass Spectrom. 2010, 24, 1147-1154.

239. Cha, S. W.; Song, Z. H.; Nikolau, B. J.; Yeung, E. S., Direct Profiling and Imaging of Epicuticular Waxes on Arabidopsis Thaliana by Laser Desorption/Ionization Mass Spectrometry Using Silver Colloid as a Matrix. Anal. Chem. 2009, 81, 2991-3000.

240. Sun, Z.; Findsen, E. W.; Isailovic, D., Atmospheric Pressure Visible-Wavelength MALDI-MS. Int. J. Mass spectrom. 2012, 315, 66-73.

241. Cheng, S. C.; Huang, M. Z.; Wu, L. C.; Chou, C. C.; Cheng, C. N.; Jhang, S. S.; Shiea, J., Building Blocks for the Development of an Interface for High-Throughput Thin Layer Chromatography/Ambient Mass Spectrometric Analysis: A Green Methodology. Anal. Chem. 2012, 84, 5864-5868.

242. Hwang, D. J.; Grigoropoulos, C. P.; Yoo, J.; Russo, R. E., Optical Near-Field AblationInduced Plasma Characteristics. Appl. Phys. Lett. 2006, 89, 254101.

243. Little, M. W.; Laboy, J.; Murray, K. K., Wavelength Dependence of Soft Infrared Laser Desorption and Ionization. J. Phys. Chem. C 2007, 111, 1412-1416.

244. Murray, K. K.; Ghorai, S.; Seneviratne, C. A., Tip Enhanced Laser Ablation Sample Transfer for Mass Spectrometry. MRS Proc. 2015, 1754, mrsf14-1754-pp08-04.

245. Römpp, A.; Schäfer, K. C.; Guenther, S.; Wang, Z.; Köstler, M.; Leisner, A.; Paschke, C.; Schramm, T.; Spengler, B., High-Resolution Atmospheric Pressure Infrared Laser Desorption/Ionization Mass Spectrometry Imaging of Biological Tissue. Anal. Bioanal. Chem. 2013, 405, 6959-6968. 
246. Jackson, S. N.; Wang, H.-Y. J.; Woods, A. S., In Situ Structural Characterization of Phosphatidylcholines in Brain Tissue Using MALDI-MS/MS. J. Am. Soc. Mass Spectrom. 2005, 16, 2052-2056.

247. Jackson, S. N.; Wang, H.-Y. J.; Woods, A. S.; Ugarov, M.; Egan, T.; Schultz, J. A., Direct Tissue Analysis of Phospholipids in Rat Brain Using MALDI-TOFMS and MALDI-Ion Mobility-TOFMS. J. Am. Soc. Mass Spectrom. 2005, 16, 133-138.

248. Marto, J. A.; White, F. M.; Seldomridge, S.; Marshall, A. G., Structural Characterization of Phospholipids by Matrix-Assisted Laser Desorption/Ionization Fourier Transform Ion Cyclotron Resonance Mass Spectrometry. Anal. Chem. 1995, 67, 3979-3984.

249. Barenholz, Y.; Thompson, T. E., Sphingomyelin: Biophysical Aspects. Chem. Phys. Lipids 1999, 102, 29-34.

250. Petković, M.; Schiller, J.; Müller, M.; Benard, S.; Reichl, S.; Arnold, K.; Arnhold, J., Detection of Individual Phospholipids in Lipid Mixtures by Matrix-Assisted Laser Desorption/Ionization Time-of-Flight Mass Spectrometry: Phosphatidylcholine Prevents the Detection of Further Species. Anal. Biochem. 2001, 289, 202-216.

251. DiResta, G.; Lee, J.; Lau, N.; Ali, F.; Galicich, J.; Arbit, E., Measurement of Brain Tissue Density Using Pycnometry. In Brain Edema VIII, Springer: 1990; pp 34-36.

252. Guez-Barber, D.; Fanous, S.; Harvey, B. K.; Zhang, Y.; Lehrmann, E.; Becker, K. G.; Picciotto, M. R.; Hope, B. T., Facs Purification of Immunolabeled Cell Types from Adult Rat Brain. J. Neurosci. Methods 2012, 203, 10-18.

253. Zeisel, A.; Muñoz-Manchado, A. B.; Codeluppi, S.; Lönnerberg, P.; La Manno, G.; Juréus, A.; Marques, S.; Munguba, H.; He, L.; Betsholtz, C., Cell Types in the Mouse Cortex and Hippocampus Revealed by Single-Cell RNA-Seq. Science 2015, 347, 11381142.

254. Mishra, S.; Yadava, V., Laser Beam Micromachining (LBMM)-a Review. Opt. Laser. Eng. 2015, 73, 89-122.

255. Park, S. G.; Murray, K. K., Laser Ablation Sample Transfer for Mass Spectrometry Imaging. In Mass Spectrometry Imaging of Small Molecules, Springer: 2015; pp 129-139.

256. Zorba, V.; Mao, X.; Russo, R. E., Optical Far-and Near-Field Femtosecond Laser Ablation of Si for Nanoscale Chemical Analysis. Anal. Bioanal. Chem. 2010, 396, 173180 . 
257. Zenobi, R.; Deckert, V., Scanning Near-Field Optical Microscopy and Spectroscopy as a Tool for Chemical Analysis. Angew. Chem. Int. Ed. 2000, 39, 1746-1756.

258. Hecht, B.; Sick, B.; Wild, U. P.; Deckert, V.; Zenobi, R.; Martin, O. J.; Pohl, D. W., Scanning Near-Field Optical Microscopy with Aperture Probes: Fundamentals and Applications. J. Chem. Phys. 2000, 112, 7761-7774.

259. Dutta Majumdar, J.; Manna, I., Laser Material Processing. Int. Mater. Rev. 2011, 56, 341388.

260. Huang, S.; Hong, M.; Luk'yanchuk, B.; Zheng, Y.; Song, W.; Lu, Y.; Chong, T., Pulsed Laser-Assisted Surface Structuring with Optical Near-Field Enhanced Effects. J. Appl. Phys. 2002, 92, 2495-2500.

261. Lu, Y.; Chen, S., Nanopatterning of a Silicon Surface by Near-Field Enhanced Laser Irradiation. Nanotechnology 2003, 14, 505.

262. Danilevičius, P.; Rekštytè, S.; Balčiūnas, E.; Kraniauskas, A.; Širmenis, R.; Baltriukienė, D.; Bukelskiené, V.; Gadonas, R.; Sirvydis, V.; Piskarskas, A., Laser 3d Micro/Nanofabrication of Polymers for Tissue Engineering Applications. Opt. Laser Technol. 2013, 45, 518-524.

263. Thompson, M., Handbook of Inductively Coupled Plasma Spectrometry; Springer Science \& Business Media, 2012.

264. Moore, G. L., Introduction to Inductively Coupled Plasma Atomic Emission Spectrometry; Elsevier, 2012.

265. Sabine Becker, J., Imaging of Metals in Biological Tissue by Laser Ablation Inductively Coupled Plasma Mass Spectrometry (La-Icp-MS): State of the Art and Future Developments. J. Mass Spectrom. 2013, 48, 255-268.

266. Zeisel, D.; Dutoit, B.; Deckert, V.; Roth, T.; Zenobi, R., Optical Spectroscopy and Laser Desorption on a Nanometer Scale. Anal. Chem. 1997, 69, 749-754.

267. Novotny, L.; Hecht, B., Principles of Nano-Optics; Cambridge University Press, 2012.

268. Imura, K.; Nagahara, T.; Okamoto, H., Near-Field Two-Photon-Induced Photoluminescence from Single Gold Nanorods and Imaging of Plasmon Modes. J. Phys. Chem. B 2005, 109, 13214-13220.

269. Novotny, L.; Bian, R. X.; Xie, X. S., Theory of Nanometric Optical Tweezers. Phys. Rev. Lett. 1997, 79, 645. 
270. Hommelhoff, P.; Sortais, Y.; Aghajani-Talesh, A.; Kasevich, M. A., Field Emission Tip as a Nanometer Source of Free Electron Femtosecond Pulses. Phys. Rev. Lett. 2006, 96, 077401 .

271. Lee, M.; Reifenberger, R.; Robins, E.; Lindenmayr, H., Thermally Enhanced Field Emission from a Laser - Illuminated Tungsten Tip: Temperature Rise of Tip. J. Appl. Phys. 1980, 51, 4996-5006.

272. Furukawa, H.; Kawata, S., Local Field Enhancement with an Apertureless Near-FieldMicroscope Probe. Opt. Commun. 1998, 148, 221-224.

273. Link, S.; El-Sayed, M. A., Size and Temperature Dependence of the Plasmon Absorption of Colloidal Gold Nanoparticles. J. Phys. Chem. B 1999, 103, 4212-4217.

274. Vella, A.; Sevelin-Radiguet, N.; Houard, J.; Deconihout, B., Polarization Dependence of the Optical Absorption of a Subwavelength Tip. Appl. Surf. Sci. 2012, 258, 9202-9207.

275. Harrison, R.; Ben-Yakar, A., Role of Near-Field Enhancement in Plasmonic Laser Nanoablation Using Gold Nanorods on a Silicon Substrate. Opt. Express 2010, 18, 22556-22571.

276. Robitaille, A.; Boulais, É.; Meunier, M., Mechanisms of Plasmon-Enhanced Femtosecond Laser Nanoablation of Silicon. Opt. Express 2013, 21, 9703-9710.

277. Dixon, J. M.; Taniguchi, M.; Lindsey, J. S., Photochemcad 2: A Refined Program with Accompanying Spectral Databases for Photochemical Calculations. Photochem. Photobiol. 2005, 81, 212-213.

278. Krug II, J. T.; Sánchez, E. J.; Xie, X. S., Design of Near-Field Optical Probes with Optimal Field Enhancement by Finite Difference Time Domain Electromagnetic Simulation. J. Chem. Phys. 2002, 116, 10895-10901.

279. Nemes, P.; Vertes, A., Ambient Mass Spectrometry for in Vivo Local Analysis and in Situ Molecular Tissue Imaging. TrAC Trend. Anal. Chem. 2012, 34, $22-34$.

280. Rezenom, Y. H.; Dong, J.; Murray, K. K., Infrared Laser-Assisted Desorption Electrospray Ionization Mass Spectrometry. Analyst 2008, 133, 226-232.

281. Grada, A.; Weinbrecht, K., Next-Generation Sequencing: Methodology and Application. J. Invest. Dermatol. 2013, 133, e11. 


\section{APPENDIX. LETTERS OF PERMISSION}

$4 / 14 / 2016$
RightsLink Printable License

SPRINGER LICENSE

TERMS AND CONDITIONS

Apr 14, 2016

This is a License Agreement between Chinthaka A Seneviratne ("You") and Springer ("Springer") provided by Copyright Clearance Center ("CCC"). The license consists of your order details, the terms and conditions provided by Springer, and the payment terms and conditions.

All payments must be made in full to $\mathrm{CCC}$. For payment instructions, please see information listed at the bottom of this form.

License Number

License date

Licensed content publisher

Licensed content publication

Licensed content title

Licensed content author

Licensed content date

Volume number

Issue number

Type of Use

Portion

Number of copies
3847740463894

Apr 14, 2016

Springer

Journal of The American Society for Mass Spectrometry

Tip-Enhanced Laser Ablation Sample Transfer for Biomolecule Mass Spectrometry

Suman Ghorai

Jan 1,2014

26

1

Thesis/Dissertation

Full text

1

Author of this Springer article Yes and you are a contributor of the new work

Order reference number None

Title of your thesis /

Tip-Enhanced Laser Ablation Sample Transfer for Mass Spectrometry

dissertation

Expected completion date

May 2016

Estimated size(pages)

112

Total

0.00 USD

Terms and Conditions

Introduction

The publisher for this copyrighted material is Springer. By clicking "accept" in connection with completing this licensing transaction, you agree that the following terms and conditions apply to this transaction (along with the Billing and Payment terms and conditions established by Copyright Clearance Center, Inc. ("CCC"), at the time that you opened your Rightslink account and that are available at any time at http://myaccount.copyright.com). Limited License

With reference to your request to reuse material on which Springer controls the copyright, permission is granted for the use indicated in your enquiry under the following conditions: 


\section{CAMBRIDGE UNIVERSITY PRESS LICENSE TERMS AND CONDITIONS}

This Agreement between Chinthaka A Seneviratne ("You") and Cambridge University Press ("Cambridge University Press") consists of your license details and the terms and conditions provided by Cambridge University Press and Copyright Clearance Center.

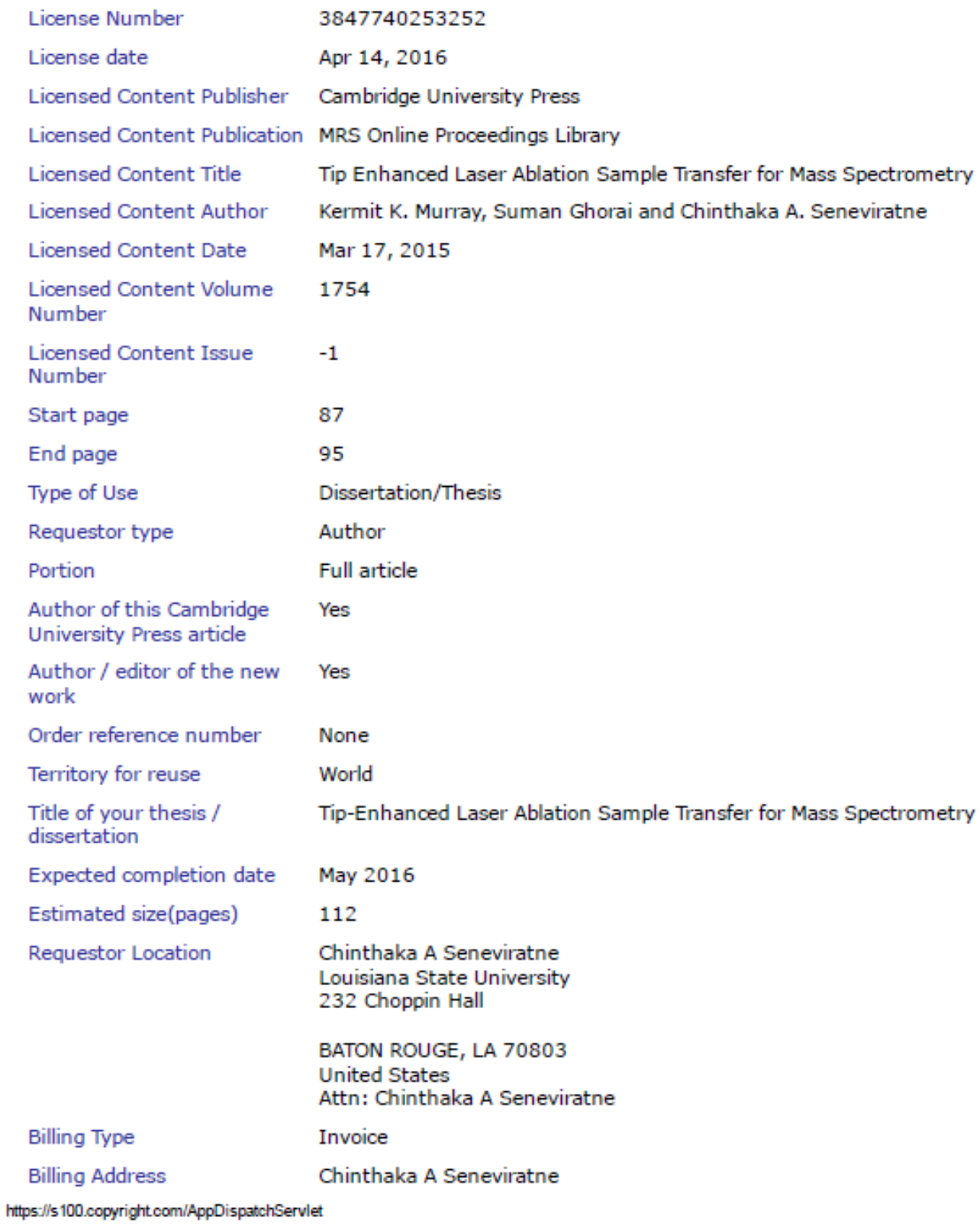


Terms and Conditions

\section{TERMS \& CONDITIONS}

Cambridge University Press grants the Licensee permission on a non-exclusive nontransferable basis to reproduce, make available or otherwise use the Licensed content 'Content' in the named territory 'Territory' for the purpose listed 'the Use' on Page 1 of this Agreement subject to the following terms and conditions.

1. The License is limited to the permission granted and the Content detailed herein and does not extend to any other permission or content.

2. Cambridge gives no warranty or indemnity in respect of any third-party copyright material included in the Content, for which the Licensee should seek separate permission clearance.

3. The integrity of the Content must be ensured.

4. The License does extend to any edition published specifically for the use of handicapped or reading-impaired individuals.

5. The Licensee shall provide a prominent acknowledgement in the following format: author/s, title of article, name of journal, volume number, issue number, page references, , reproduced with permission.

Other terms and conditions:

v1.0

Questions? customercare@copyriaht.com or +1-855-239-3415 (toll free in the US) or +1-978-646-2777. 


\section{VITA}

Chinthaka Aravinda Seneviratne was born in Chilaw, Sri Lanka, in 1982. After the successful completion of the General Certificate of Education Advanced Level examination (GCE A/L), he was selected to pursue a Bachelor of Science, at the University of Kelaniya (UOK), Sri Lanka. He graduated from the UOK, in 2008, where he received a special degree in chemistry. At UOK, he conducted a research to identify the origin of Sri Lankan tea using phytochemical markers under the supervision of Professor Kapila N. Seneviratne. After receiving his BS degree, he worked as a teaching assistant in the Department of Chemistry at UOK for one year and he had decided to extend his studies in the USA. In August 2009 he enrolled in the chemistry graduate program at Bucknell University in Lewisburg Pennsylvania. There, he chose to work in Professor, Timothy G. Strein's research group, where he began developing methods to detect creatinine in body fluids using capillary electrophoresis. In 2011, he entered the doctoral program for chemistry at Louisiana State University. His research mentor at Louisiana State University was Professor. Kermit K. Murray. He is a member of the American Society for Mass Spectrometry and the American Chemical Society. Mr. Seneviratne is currently a candidate for the degree of Doctor of Philosophy in chemistry, which will be awarded at the May 2016 Commencement. 